\title{
THE AMERICAN OOLOGISTS
}

\section{EXCHANGE PRICE LIST}

OF

NORTH AMERICAN BIRDS' EGGS

QL

1922

675

A512

1922

BIRDS

COMPILED BY

A COMMITTEE OF TWENTY-FIVE

PROMINENT AMERICAN OOLOGISTS

PUУLISHED BY

R. MAGOON BARNES

LACON. ILL.. U.S. A. 





\section{"THE OOLOGIST"}

Is the only publication in the Western Hemisphere, devoted to Oology and the interests of Oologists.

Its contributors are the best students of Ornithology and collectors of eggs. It is not too scientific not to be popular in style and not too popular not to be scientific in accuracy.

Its columns teem with advertisements of Bird Skins, Nests and Eggs as well as Living Specimens. It is of the greatest assistance to those who desire to sell or exchange specimens, and its advertising columns are consulted by nearly all the large museums and leading collectors in the world.

Published by R. MAGOON BARNES

SUBSCRIPTION

FIFTY CENTS per year in advance.

ADDRESS

THE OOLOGIST, LACON, ILL. 


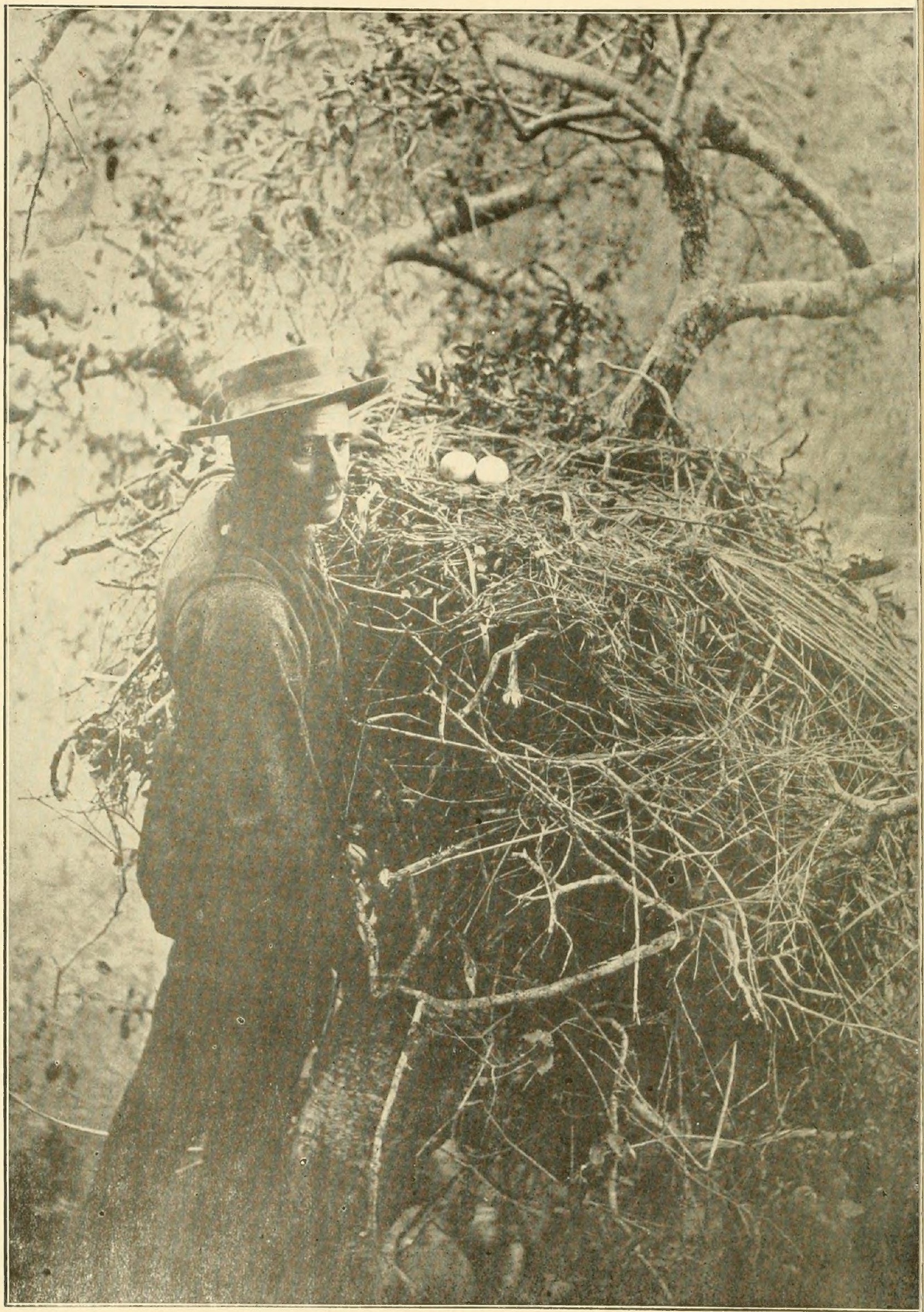

R. M. Barnes at Golden Eagle's nest, Escadido, Calif., March 30, 1912 


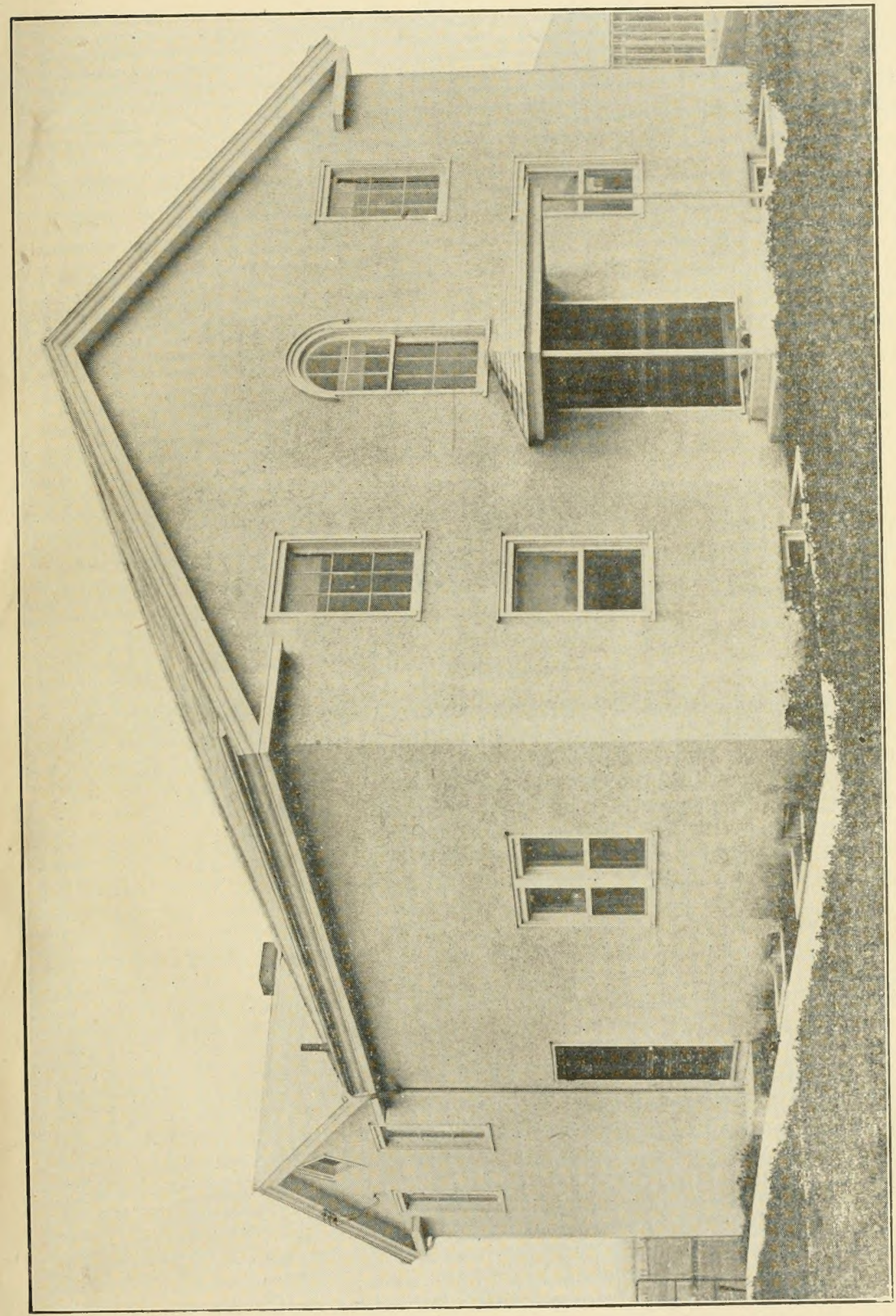

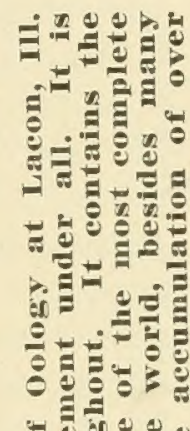

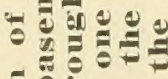

引

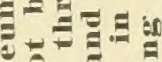

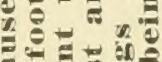

$\exists=$

$\infty$

is

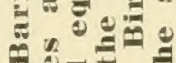

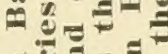

ह

o $\leqslant \div$

暗

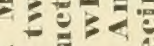

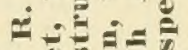

每

in

9. 10,2 :

$\exists .2 \div$

的的

(1)

-

政

( 


\section{INTRODUCTION}

\section{BIRDS' EGGS}

\section{ALWAYS COLLECTED FOR STUDY AND BEAUTY}

Since the earliest activities of man of which we have any reliable historic record, the wonders and mysteries of nature have appealed to the greatest minds among men. Artists have painted, poets have sung, and scientists have written of the marvels which surround us on every hand Collectors in all fields of natural history research have gathered together wccumulations of the objects of nature; for study, comparison, and exhibition. Perhaps the most marvelous of nature's mysteries relate to the reproduction of animate life. And one of the methods employed by nature, is to wrap the life spark of the family of Aves, (Birds) up within a hard partially porous shell composed largely of lime, with a generous supply of food for the young bird; and to cause this life spark to germinate during a period of incubation, which varies in length with different birds, during which in most cases, the egg is kept warm by the mother bird sitting on it. At the end of this period of incubation the young bird hatches, that is breaks out of the shell.

The size, shape, color, markings and texture of the shells of birds' eggs is of infinite variety, and their beauty is proverbial. These facts early attracted the students of nature. Being easy to preserve these shells when properly prepared, lasting for an almost endless time, if properly protected, and presenting in the collector's cabinets a beautiful sight, birds' eggs early attracted the collectors of natural history specimens.

\section{CATALOGUES}

In the course of time those collecting in different parts of the country commenced to exchange specimens with each other, and in time some persons began to deal commercially in eggs, or rather the shells of eggs, and it became the custom of such dealers to issue catalogues or price lists. Most of these were inspired by arranging the prices so as to get the most profit for the dealers. At times lists were issued for the purpose of equalizing the prices of specimens from the different parts of the country. Still others were issued apparently for the purpose of giving one part of the country an advantage over other parts in the matter of prices. Various concerns issued their lists in times past. F. T. Pember of Granville, N. Y., in 1885. F. H. Lattin of Gaines, N. Y, in 1884. E. H. Short of Albion, N. Y., in 1892 and 1905. Southwick \& Jenks of Providence, R. I.; Chas. J. Maynard, of Boston; F. B. Webster \& Co., of Hyde Park, Mass., issued lists at various times; and Harry R. Taylor of Almeda, Calif., in 1907, issued the last general American price list. All of these lists gradually became obsolete.

The last generally accepted revision of prices for North American Birds' Eggs was in 1905, though an alleged revision in 1915 was advertised, and a few copies sold under misleading advertisements, but it was never received or accepted by the body of American Oologists as anything but a' miscarriage.

The need of a general overhauling of Exchange prices become imperative. Years had passed and birds once common have become scarce, and in some instances extinct Many birds whose nesting places were unknown a few years a'go, have been discovered in their summer homes. Others whose breeding was in distant lands, or little known places, have been brought into closer touch with the collector, by the railway, the motor vehicle and the penetration of far away places by steam and motor boat.

Many eggs formerly very rare because the owners nested in distant places have become common, because the settlers have occupied their home territory, and brought them in common contact with the collector This great change has worked both ways, enchancing the value of some specimene and markedly decreasing the value of others 


\section{HOW THIS LIST CAME INTO EXISTENCE}

Therefore as it became apparent that the old price lists had become of little use as a ba'sis on which to exchange specimens, the undersigned through the columns of "The Oologist," Vol. XXXVI No. 2, Page 34, February 1919, called an election to be participated in by all the Oologists of North America, for the purpose of electing a committee of twenty-five recognized Oologists to revise the exchange prices of North American birds' eggs.

The call for this election was issued with the express announcement that we would not accept any election to this committee nor any appointment to the final supreme committee of review. And that when the committee were ready to report their a'ction on prices we would publish the catalogue at actual cost.

For this purpose there were created five areas. These areas and the number of committeemen to be elected from each were as follows:

Pacific Coast Area. Three Committeemen, from California, Oregon and Washington.

Intra-Mountain Area. Three Committeemen, from Arizona, Colorado, Idaho, Montana, Nevada, New Mexico, Utah, Wyoming.

Western Area. Three Committeemen, from Arkansas, Kansas, Minnesota, Nebraska, North Dakota, Oklahoma, South Dakota, Texas.

Central West Area. Three Committeemen, from Illinois, Indiana, Iowa, Michigan, Missouri, Ohio and Wisconsin.

Central East Area. Four Committeemen, from Delaware, Maryland, Pennsylvania, New Jersey, New York, West Virginia.

New England Area. Three Committeemen, from Connecticut, Maine, Massachusetts, New Hampshire, Rhode Island and Vermont.

Southern Area. Three Committeemen, from Alabama, Georgia, Florida, Kentucky, Louisiana, Mississippi, North Carolina, South Carolina, Tennessee, Virginia.

Canadian Area. Three Committeemen, from Canada:

This percentage of committeemen was based on the known number of active Oologists within each area:

It was also provided that after each sub-committee from each of the above areas had reported the prices that such sub-committee had agreed upon for all of the country; then a supreme Committee of Three should take the separate lists from ewch area and pass as a final committee on the prices to appear in the catalogue. We are informed that this final committee reached the results presented in this volume by averaging the prices on each of the several lists from the different areas A more fair way of getting at the general consensus of opinion could not have been adopted we believe.

At this election the Committee of Twenty-five whose names appear in the report of that committee printed herewith were elected. This committee labored earnestly and diligently for almost two years on the problem. The result of their deliberations being finally passed on by this supreme Committee of Three above referred to. The prices appearing in this volume are the result. 


\section{THE PRICES IN THIS CATALOGUE AND CASH VALUES}

It must be understood that these prices following are relative and not intended to represent cash values. They are to be used as a basis for the exchange of specimens, and of course refer only to specimens first class in every respect. The cash value of a bird's egg is usually a matter of agreement between the buyer and seller, where it is a cash transaction; but is usually reached, and almost invariably so in the sale of entire col'ections, on a percentage basis of the aggregate value of the specimens sold, as shown by some price list. It is our experience that these percentages range from $10 \%$ to $331-3 \%$ according to the varieties $0^{\wedge}$ the lot. Sometimes, however, these sales are made at so much per egg for the entire collection. We have purchased at different times entire collections of some of the best known American Oologists at a blanket price of ten cents per egg. At other times we have purchased selected lots of eggs from collectors who were breaking up their collections, and where we were allowed to pick out only such as we could use; these of course being the rarer specimens, at $25 \%$ of the old list prices and less. There is no such thing as a regular standard cash market price for bird's eggs. The price at which they will sell depends always on the supply and demand.

No collector should ever collect any eggs of the commoner species for any purpose except for his own collection. There is no demand for such either in exchange or cash. It would be a good rule to adopt, to collect nothing for exchange purposes, except on special order, that is priced in this catalogue, at less than 50 cents per egg.

A competent collector with a fair scientific knowledge of birds and their habits, can always make a good average salary by collecting specimens of birds, their nests and eggs for public institutions, and for private collectors who are unable for lack of time and because of business cares to do extensive field work. Such a collector who has established a reputation for integrity, can always thus finance an outing or a vacation trip into the far away wild places that he might not otherwise be unable to visit. We have often wondered why more of the younger generation of Naturalists did not take advantage of such opportunities.

The eggs of the seventy-three species unpriced in this catalogue are so rare that the committee determined there was no uniform value as to them. To secure one of these is to secure a prize indeed.

All prices given preceeded by a* are for specimens taken without the territorial limits covered by the A. O. U. List only, such as European, etc.

The following from the pen of the writer published in "The Oologist" Vol. XXXII, P. P. 78 and 134 will give the readers a fair idea of how to prepare birds' eggs for collections. 


\section{COLLECTING BIRDS' EGGS}

\section{What Is Needed-Preparing Specimens}

It is useless, even wanton, to collect eggs of our birds unless with a definite purpose. Eggs are absolutely worthless or worse unless properly collected, prepared, preserved and recorded. To do these things one must be equipped with some things, chiefest of which is a settled determination to do whatever is done well.

First of all you will want a note book in which to record your observations. Do not rely on memory for anything, the fuller and more complete the notes the better. From two to a half dozen egg drills and a blow-pipe or two are necessary. Get the "cut the lining" kind of drills of assorted sizes. These with the blow-pipes may be had of dealers in such instruments. They should be thoroughly cleansed after each using. Some sort of a receptacle, a cigar box filled with cotton or something of the kind is necessary to carry eggs in while afield and a safe and secure one in which to keep them after coming home.

A copy of the American Ornithologists List (The A. O. U. List) and either "Bailey's Birds of the Western United States," or Cha'pman's "Birds of Eastern North America" will be well nigh indispensable. Likewise Charles K. Reed's "North American Birds Eggs" or Oliver Davies' "Nests and Eggs of North American Birds" will be found of some help.

Assuming you have some or all of the above let us start out some bright spring morning in quest of specimens. The sun is bright and warm; there is a slight balmy south wind. Spring is wearing her splendid garb of fresh bright green, and her brow is garlanded with bloom of riotous color, while perfume floats in the air. With a collector's box full of cotton slung over our shoulder and our note-book in our pocket we start out, full of anticipation and life.

We know the Woodpeckers nest in holes, and Meadowlarks on the ground while Robins in trees and Indigo Buntings in bushes. We are starting a collection and of course begin with the common varieties.

Suppose we find a Robin's nest in an apple tree. Here is what we do, or should do. First make absolutely sure it is a Robin-of course we know a Robin, but later we may find some nests where we do not know the birds. So begin right now to look and be certain of the identity of the owner of the nest. Then we climb up and peep into the nest. Only two eggs! An incomplete set so we leave them and pass on.

Next we find a Blue Bird's nest in an abandoned Woodpecker's hole in a small dead limb of an apple tree. We are first very sure it is a Blue Bird. The identity of the bird is ALWAYS first. Then we look into the nest. Five fresh eggs! Good! Our first specimen for "our collection." How shall we get them out? Our hand is too large to get into the hole. It is not so very far to the house and we go back and borrow a saw. Slowly and very carefully we saw the small limb off below the nest and lower it to the ground, turn it slightly over and take a spoon out of our pocket, and lift out the beatuiful blue eggs one at a time. These we roll separately in cotton and place in our cigar box. Then we take our note-book out and sit down and enter the following:

“(1) $766 \mathrm{~A}-5$ or a $/ 5$. Nest 7 feet up in an abandoned Woodpecker's hole in dead limb of an apple tree in an orchard. Birds seen, eggs fresh, nest saved. Remarks. This nest was on the south side of the tree and the opening was toward the southeast. The cavity was 9 inches deep and the opening $21 / 4$ inches across. Nest typical, of fine grasses, weeds and feathers."

Then we replace the note-book in our pocket and place the figure (1) on the nest stub, stand it up against the foot of the tree where we can get it when we return home, and pass on.

Next we find a Phoebe's nest under a bridge across the road, stuck onto one of the projecting rocks of the retaining walls. The bird is gone like a flash but we know her. The nest contains six eggs, a very unusual number, but they show signs of incubation, however we decide to chance it, so carefully remove the eggs, and as with the Blue Bird's eggs, roll each one carefully in cotton and place them in our collecting box. Then, after removing the nest and wrapping it in a newspaper cornu copia and marking it (2), we enter in our note-book: 
"(2) 456 A-6 or a/6. Nest stuck on a projecting stone of a retaining wall of a bridge across the Lacon and Henry road 5 miles north of Lacon, 7 feet above the creek bed and 3 feet from bridge floor. Birds seen. Nest of rootlets, moss, fine grass and mud, lined with hair and feathers. Eggs, six, a very unusually large number; incubation commenced. Nest saved."

We pass on and find a Kingfisher's nest in a hole in the cut bank of this same creek. As the birds fly about we admit we are for sure stumped. We remember reading in one of Wood's books that the nest of this bird was almost untakable in it's natural state, and likewise have a vivid recollection of reading in THE OOLOGIST, Vol XXVI, page 92 of the tragic death of Richard Smithwick, who dug into a bank after a nest of this species, and crawled into the hole he dug when the earth caved in on him and smothered him. But we must have these eggs! Are we not forming a collection of eggs and we have no Kingfisher's eggs yet. Well, we go to a nearby farm house and borrow a spade. As luck would have it the nest was not over three feet below the top so we scramble up to the nest hole and insert an arm full length without finding anything but air. Then we get a small switch and push it in as far as possible with the same result. This makes fully six.feet of the tunnel we liave explored. Then we go up on top and dig down to the burrow. To our surprise we find it at two feet and four inches, showing it slopes upward. After cleaning away the dirt and digging almost two times as much as was really necessary in the hot sun, we get where we can see the eggs. Seven of them at the enlarged end of this nine foot tunnel, lying there on the soil with only a few straws about them. Disappointed? Yes! Where is the beautifully fashioned and delicately assembled nest of white fish bones and scales that I liave been taught to expect? A myth? Yes, and nothing more. 'Then the eggs were not as white as we had expected Or are they dirty? Yes, and they, like Woodpeckers' eggs, must be most carefully cleaned both inside and out of all dirt, foreign matter and the last vistage of contents with perfectly clear water, else the taking of them is in vain They will surely spot and blotch and ultimately become entirely ruined unless this care is used in preparing them. We pack these specimens, with more care and more cotton because they are larger and heavier. Then we carefully collect the few straws composing the nest, place them in some more newspaper marked (3) and in our pocket. Then we enter in our note-book:

"(3) $390 \mathrm{~A} / 7$. Nest in a burrow 9 feet deep in the side of a creek bank 7 feet above the creek and 3 feet down from the surface in sandy soil, composed of a few straws at the enlarged end, and sloping upward. Opening 4x 3 inches. Birds seen; eggs dirty but fresh."

Then we start home. Across the fields we travel when suddenly from under foot flutters a mass of feathers. Finally it rises awing and floats away. A Meadow Lark! Looking down we see a tuft of grass with a small opening in the side. Peering in, five fresh eggs are disclosed and transferred, cottonwrapped, to our collecting box. Then we sit down beside the nest and enter in our note-book:

"501 A /5. Nest on the ground in a pasture, partially sunk into the ground, in the middle of a tuft of last year's grass, arched over and lined with finer grasses. Eggs fresh. Female flushed from nest. Nest saved."

Nest saved! Yes. But how? As we are not far from our own lome we go there, get another spade and a small box $8 \times 10$ inches and three inches deep. We carefully cut the sod around the nest and under it to the same size and depth as the box, being careful at all times not to in the least disturb the nest or grasses about it, and slip the spade under it, setting the sod with the nest into the box. All the time we are handling the whole affair most delicately, else we ruin it. And if properly and carefully done, we have preserved one of the very hardest kind of specimens,-a ground sunken nest amid vegetation.

We then go home for the day with four nests and four sets of eggs.

Having arrived home with a set of 5 Bluebird's, a set of 6 Phoebe's, a set of 7 Kingfisher and a set of 5 Meadowlark, the next thing is to prepare these specimens in such a way that they will last and make a desirable appearance when placed in tho cabinet. 
The first thing to do is to lay aside each set of eggs separately on a cloth or layer of cotton batting, something that will prevent them from rolling; then procure a basin of water, a tumbler filled with water and the blowpipes and drills we have before mentioned; sit down at a low table or on a door step or some similar place, so as to bring the basin above the object you sit upon.

We will commence now with the preparation of the set of Blue Bird's eggs. Picking up one of the specimens, look it over carefully to see if there are any stains or any foreign matter on it which cannot be removed, and if there is, that is the side we will blow it on. First pierce the shell with a fine pointed pin or needle, then select the smallest of the blow pipes, which should be No. 0, carefully insert the point of the drill in the hole left by the needle, and holding the egg between the thumb and finger of the left hand, with the larger end away from you, and the second finger under the smaller end of the egg slowly twiri the drill back and forth. with the thumb and finger of the right hand, gradually enlarging the oriface until you have drilled clear into the egg. If you have the right kind of a drill the rear end of the burr part of the drill will cut the lining as it enters the egg. If it does not do this, it will be necessary to draw the drill back until the rear of the burr comes in contact with the inner surface of the shell at the hole made by the drill. A few more twirls will then cut the lining. Then withdraw the drill, turn the egg over, still holding it between the thumb and finger of the left hand, take a small blow pipe and hold the point of the same near the oriface of the egg, turning the egg upside down over the basin of water. Then blow throw the blow pipe and the air will gradually enter the egg and force the contents out of the same hole.

After the entire contents of the egg ha's been blown out in this way, then take some water in the mouth from the tumbler of water standing nearby and blow the water through the blow pipe into the egg. Do this with some force, but not enough to destroy the egg shell. After the egg lias been blown full of water, then blow the water out of the egg. Repeat this two or three times un til the contents of the egg is thoroughly cleansed out, for any foreign matter of any kind or any of the contents of the egg that may be left in will surely stain the shell and ultimately destroy the specimen.

After this has been done and you are sure that the contents of the egg is entirely out and the inner surface of the shell thoroughly cleansed, then lay the egg upside down with the hole resting on a small piece of blotting paper. This should be clean and not ink stained.

Continue the same process with each one of the eggs in this set, laying them side by side on the blotter when the blowing is completed. They should be left in this position until they are thoroughly dry, and all the other eggs taken should be drilled and blown in exactly the same manner, using care to see to it that the sets do not become mixed, and that each different set is kept separately until marked.

If the eggs are not thoroughly cleansed, their taking and preparation will be to no purpose, for they will finally become spotted and ultimately disinterrate. It is always important to use the smallest drill possible on each egg.

The above is the proceeding for blowing all eggs which are fresh. It makes no difference whether they are the size of a goose egg or the size of a Hummingbird's egg. The same proceeding is followed from beginning to end.

Assuming now that all of the eggs we took on the day before above mentioned have been blown and arranged on the blotter as directed, and have been left there long enough to become thoroughly dry, the next thing is to mark them. The marking of specimens is one of the very important steps in their preparation. Care should be taken to mark each "set neatly, legibly and according to the system used by Oologists They should be marked with a soft, pointed lead pencil unless you are a high cla'ss expert with India Ink like E. J. Court at Washington and a few others of his kind, who are very rare indeed; but if you are then you may assay the use of India ink.

The set of Bluebird's eggs should be marked as follows: Above the blow hole on the larger end of the egg and near the blow hole should be placed neatly the figures 766, which is the number of the Bluebird in the American Ornithologist's Union ("A. O. U.") Check List of North American birds. To the 
right of the blow hole should be placed a short straight line as near as may be opposite the center of the blow hole. Above this line should be placed the letter" "a" or "A" and beneath the line the figure "5." The letter "a" indicates that fact that this was the first set of Bluebirds taken this year. Any subsequent sets should be indicated by the letters b, c, d, etc. The figure 5 beneath this line will indicate the number of eggs in the set If the set had been 4,6 or 7 the numbers 4,6 or 7 , according to the number of eggs in the set would be placed beneath this line. And beneath the blow hole should be placed numbers designating the year in which the set was taken, as 1910,1911, or 1912, or what. ever the year might be. Each egg in the set should be marked in exactly the same way.

In this way it is easy to keep track of the specimens. The A. O. U. number will at once identify the specimen, the set number "a 5" will show that it is the first set taken and that there were five eggs in it. The year number beneath the blow hole will show the year in which it was taken, and reference to the data or the original note book wculd disclose these same figures.

Having marked all of the eggs taken according to the above method, the next important thing is to make out a data card for each set. This should be on a standard data blank and give all the salient facts regarding the set shown in your note book. A sample data filled in is set forth on one of the following pages. After this has been done place them in a dark cabinet of some kind with closely fitting drawers that will exclude both the dust and light The light will cause many specimens to fade; in fact, nearly all. Dust if it settles upon the eggs and the atmosphere then gets damp, will set up a peculiar character of fungus growth on the shell that will ultimately disfigure the eggs for all time; particularly those having solid color, like the Bluebird, Catbird, Wood Thrush and the like.

Of course it follows that the larger the egg the easier it is to blow. Like. wise the larger the egg, the same rules apply to prepare it with as small a drill hole as possible.

Many times it will be found that the specimens taken are more or less incubated, though it is a bad practice to take incubated eggs unless they are specimens of unusual varieties. In that case of course the taking is entirely justified. The blowing of a badly incubated specimen is a matter of tedious, hard work. Frequently you will have to use an embryo hook as well as a pair of very fine, sharp pointed embryo scissors and cut the embryo within the egg through the oriface made by the drill with the scissors and drag it out, piece at a time with the embryo hook, or force small pieces of it out by inserting the point of the blow pipe entirely within the shell of the egg. To do this neatly and safely will require a larger hole in the shell and much patience and care.

It is never very satisfying to attempt to use caustic potash or any similar substance for the dissolving of the contents of an incubated specimen as is sometimes recommended, for the reason that ultimately the shell of the egg so treated will disintegrate entirely and the strong alkaline action will injure the tint and colors on the shell.

After a little practice it is not hard to become proficient in the preparation of specimens of this kind, and you will discover that your standing and rank as an oologist will depend very largely upon the character of your work in preparing your specimens. Accuracy, neatness and cleanliness above all things should be your motto.

The following illustrations will give an idea of the more advanced method; of arranging specimens in the cabinet. Each set should be placed in a separate tray white card board one half inch deep, in which should be a layer of $a b$. sorbent cotton to fit the tray neatly. These trays should be from two inches square in multiples on up to $8 \times 16$ inches. 


\section{REPORT OF THE COMMITTEE}

For several years there has been a growing need for a new catalog of North American birds' eggs with a revision of the prices used as a basis of exchange.

Realizing this need, early in the year 1919, Judge R. M. Barnes, editor of The Oologist, proposed that a committee of twenty-five of the leading oologistis of North America be elected by the oologists at large to perform this revision More than one hundred votes were received and the following were elected as members of "The Committee of Twenty-five":

Pacific Area:

J. Hooper Bowles, Tacoma, Wash.

H. W. Carriger, Oakland, Calif.

W. L. Dawson, Santa Barbara, Calif.

Intra-Mountain Area:

A. O. Treganza, Salt Lake City, Utah

E. F. Pope, Albuquerque, New Mexico

F'. C. Willard, Farmingdale, L. I., N. Y.

Western Area:

Rev. P. B. Peabody, Blue Rapids, Kansas

Rev. H. E. Wheeler, Fayetteville, Arkansas

Guy Love, Oberlin, Kansas

Central Western Area:

A. E. Price, Grant Park, Ill.

G. A. Abbott, Tulsa, Okla.

Dr. B, R. Bales, Circleville, Ohio.

Eastern Area:

Hon. J Parker Norris, Philadelphia, $\mathrm{Pa}$.

R. C. Harlow, State College, Pa.

T. H. Jackson, West Chester, Pa.

E. H. Short, Rochester, N. Y.

New England Area:

Col. John E. Thayer, Lancaster, Mass.

A. C. Bent, Taunton, Mass.

Roscoe I. Giles, Marlboro, Mass.

Southern Area:

Troup D. Perry, Savannah, Ga.

O. E. Baynard, Plant City, Fla.

H. H. Bailey, Newport News, Va.

Canadian Area:

Edward Arnold, Montreal, Quebec

R. W. Tufts, Wolfville, Nova Scotia

Walter Raine, Toronto, Ontario

A circular letter sent to the members of this committee by W. L. Dawson, from the Museum of Comparative Oology, called for an election of officers and offered several timely suggestions. The election of officers resulted as follows: Honorary President, A. C. Bent; Chairman, Dr. B R. Bales, Secretary, Rev. H. E. Wheeler.

By an overwhelming vote, it was decided not to quote birds' eggs at a market cash price, but "to express the rarity of birds' eggs in terms of dollars and cents." It was also decided that each member of the committee should work independently and send the results of his labor to the chairman of his particular area who would make an average for the area and forward the result to a "Committee on Final Values," to be anpointed by the chairman of the "Committee of Twenty-five." 
The chairman appointed this very important "Committee of Final Values" as follows: Chairman, J. Hooper Bowles, to look after the interests of the Western oologists, Richard ('. Harlow, to perform a like service for those of the East, and Frank ('. Willard, now of the East, but formerly of the West, whose sympathies would be with both.

After almost two years, the results from the various areas finally came into the hands of the "Committee on Final Values" who, as previously agreed upon, added the sum of the eight areas and divided the sum by eight; hence the exchange price set opposite the name of any species, represents the average of all the prices for this species furnished by the "Committee of Twenty-five."

It was agreed that no egg should be priced lower than ten cents, it being considered that it was worth at least that much to prepare an egg nicely and write the set data.

The committee feels that it has furnished the oologists of North America a catalog honestly prepared, with fairness to all and special favors to none.

The committee also feels that the North American oologists will appreciate a catalog prepared in this manner, although it is inevitable that there will be some dissatisfaction and honest differences of opinion regarding prices.

In closing, I think that the thanks of the whole oological fraternity are due to Messrs. Bowles, Harlow and Willard, the "Committee on Final Values" who have worked long and faithfully, often against heavy obstacles; to the secretary, Rev. H. E. Wheeler, whose heavy correspondence was of very great importance; to the many, many oologists who helped with kindly words of advice and encouragement; and last but not least, to Judge R. M. Barnes, the publisher, who has made the dream of a new catalog of North American birds' eggs come true.

B. R. BALES, M. D.,

Chairman. 


\section{Acknowledgments}

T is a duty as well as a pleasure to acknowledge the very great assistance given us by the Oological Fraternity in general in bringing the catalog into being.

To the Committee of Twenty-five who labored earnestly and long with the question of prices, and the final Committee of review, we all owe a debt of lasting gratitude.

To W. E. Clyde Todd, of The Carnegie Museum of Pittsburgh, who kindly volunteered to read proof on the scientific names, the publisher is under very special obligations for lifting the burden of responsibility from our busy shoulders.

To George Miksch Sutton, of the same institution we are likewise obligated for the splendid half-tone drawing of the eggs, illustrative of the proper method of marking.

R. M. BARNES. 



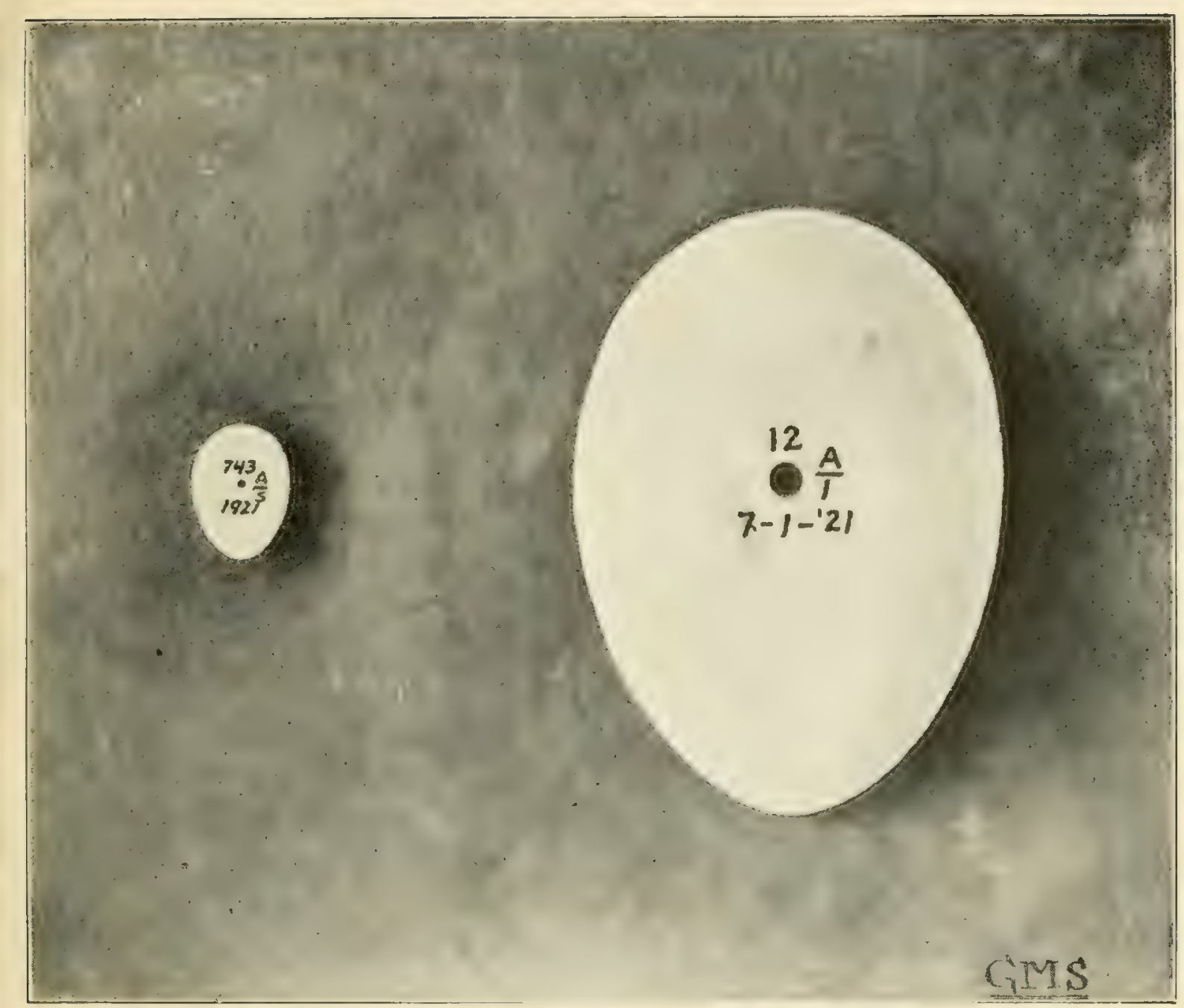

Plate showing the proper way to mark egges for the cabinet. I'lae A. O. U. No., the set mark and the date. 'These marks must correspond with the date. 'Wo mark specimens aceurately and neaty is of prime importance if they are to be accepted as first class. 


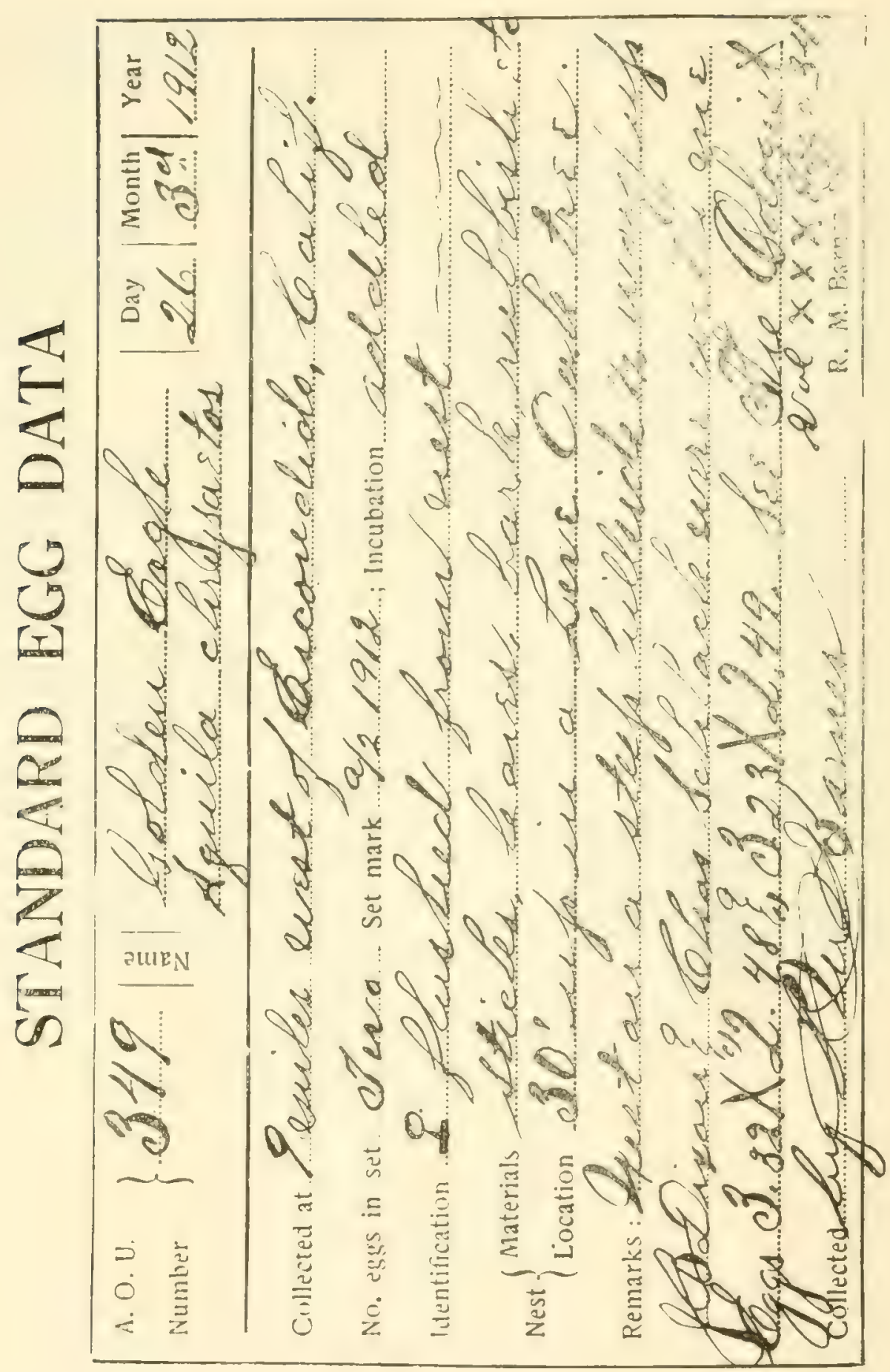


EXCHANGE PRICE LIST

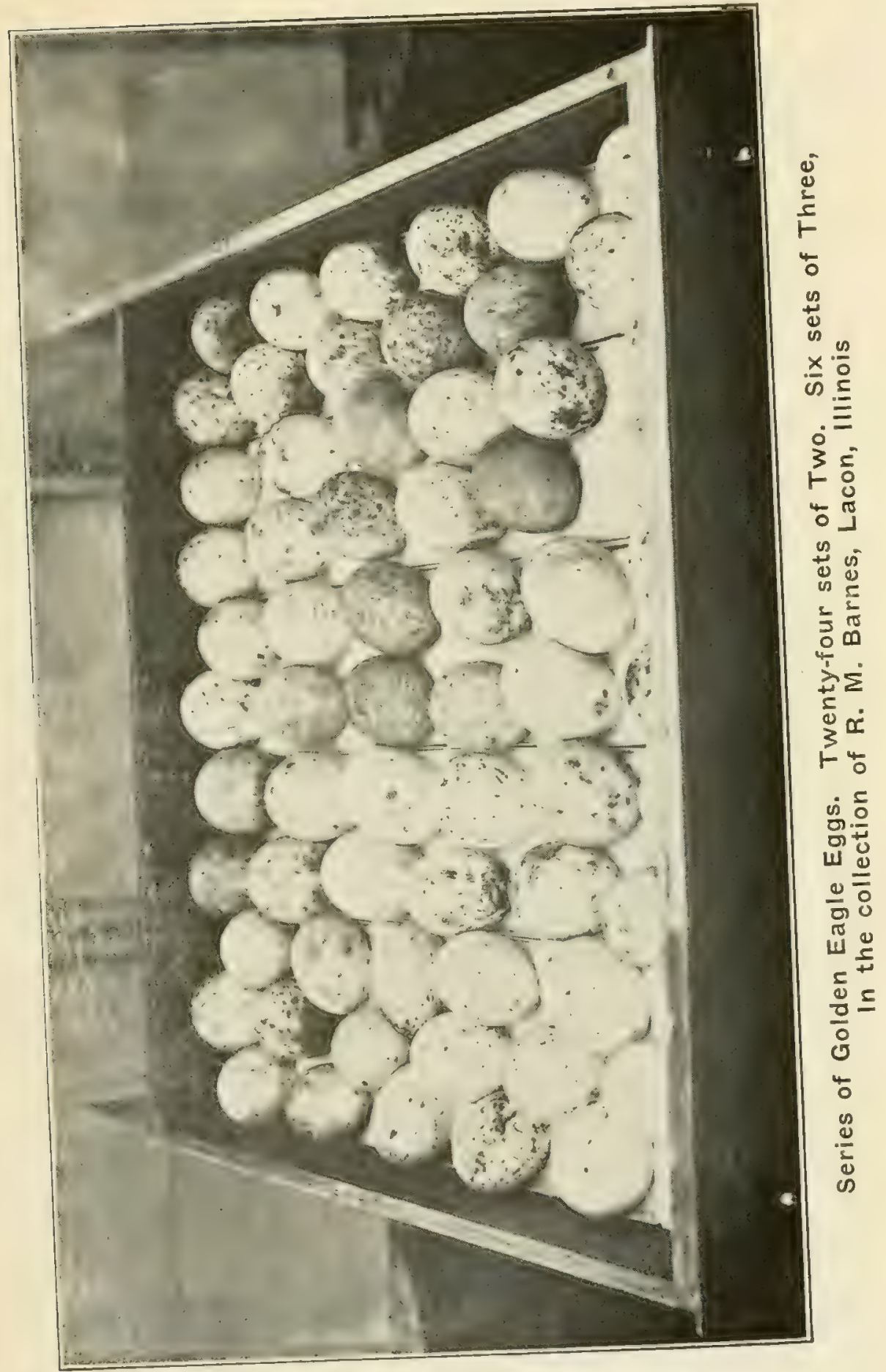




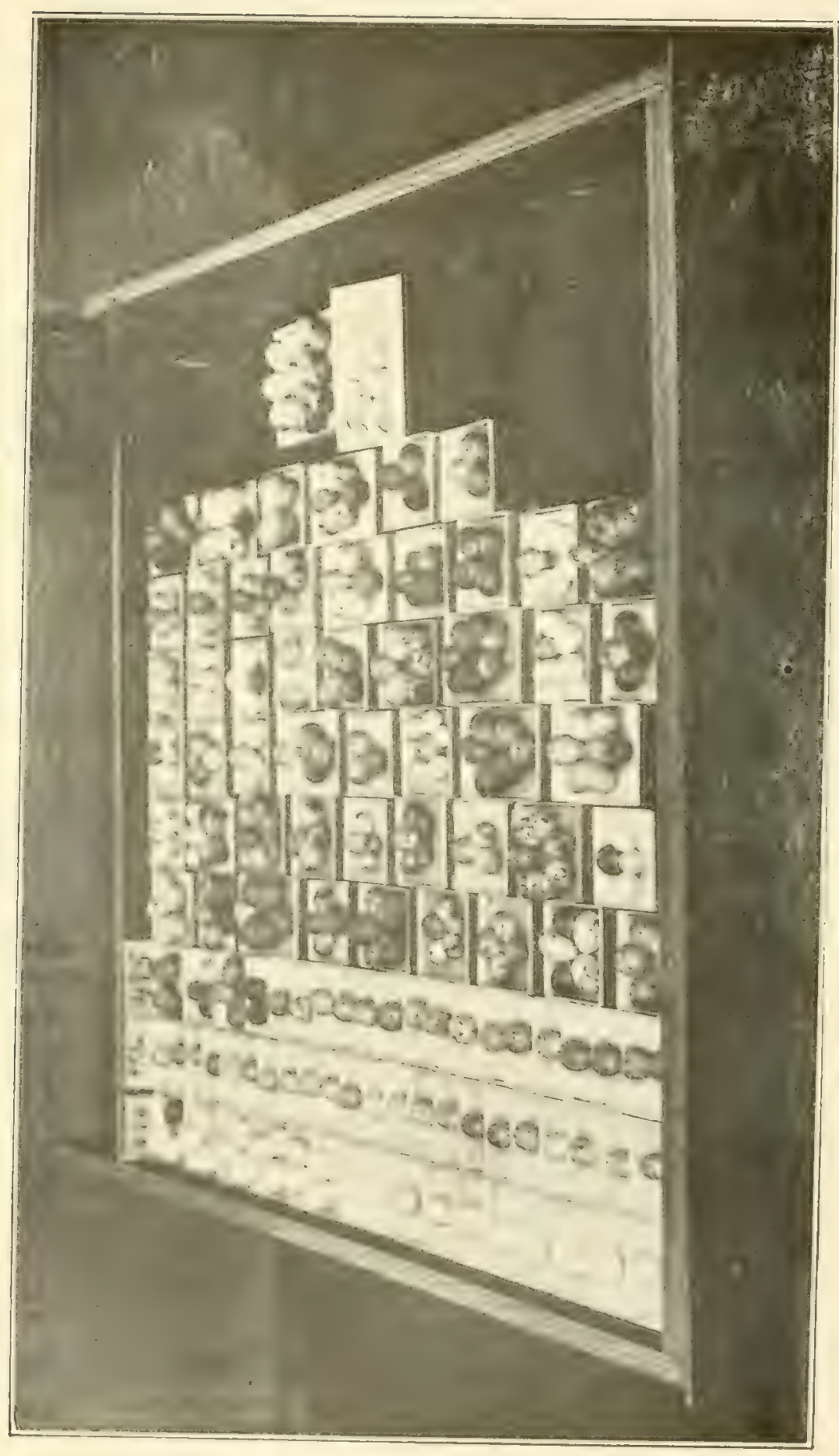

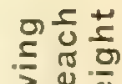

उष् 它此

范苾

is os

of

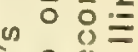
흥요을 两 $3 \cdots$ so ن० 1038 00 . ¿ (i) E $4-\frac{0}{0}$ 近 +80 4

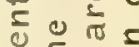
进山. Q 당 웡 ये थे

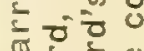
$\frac{-1}{4}$ 응 33. ¿Uல (1) $\frac{1}{0}$

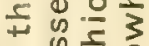
4 ㄷํㅇ은 은 운 군 ๘ 0 을 3 要 0 잉 है 동

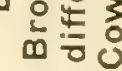




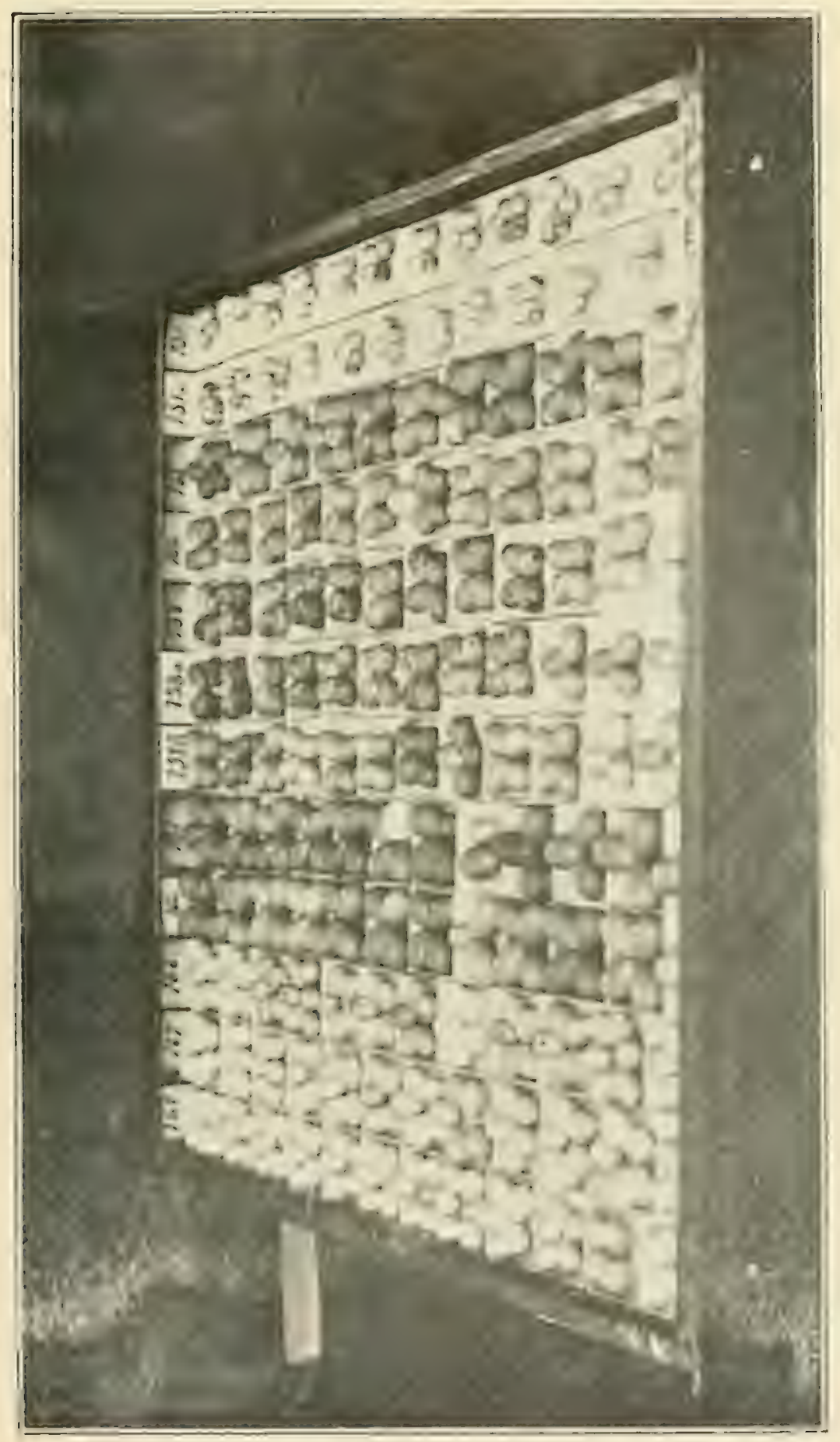



둥

동.

음

E

엉

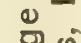

๘

$\pi \frac{\pi}{\pi}$

$\therefore$ ᄃ

m

$\because \check{c}$

ڤั

"ᄂ

$+\frac{7}{4}$

证

1.

잉

$\sum^{2}=$

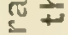

तs $\frac{10}{0}$

은

更

운 ?

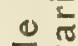

$\frac{0}{2}$

है

苟

虫

类 


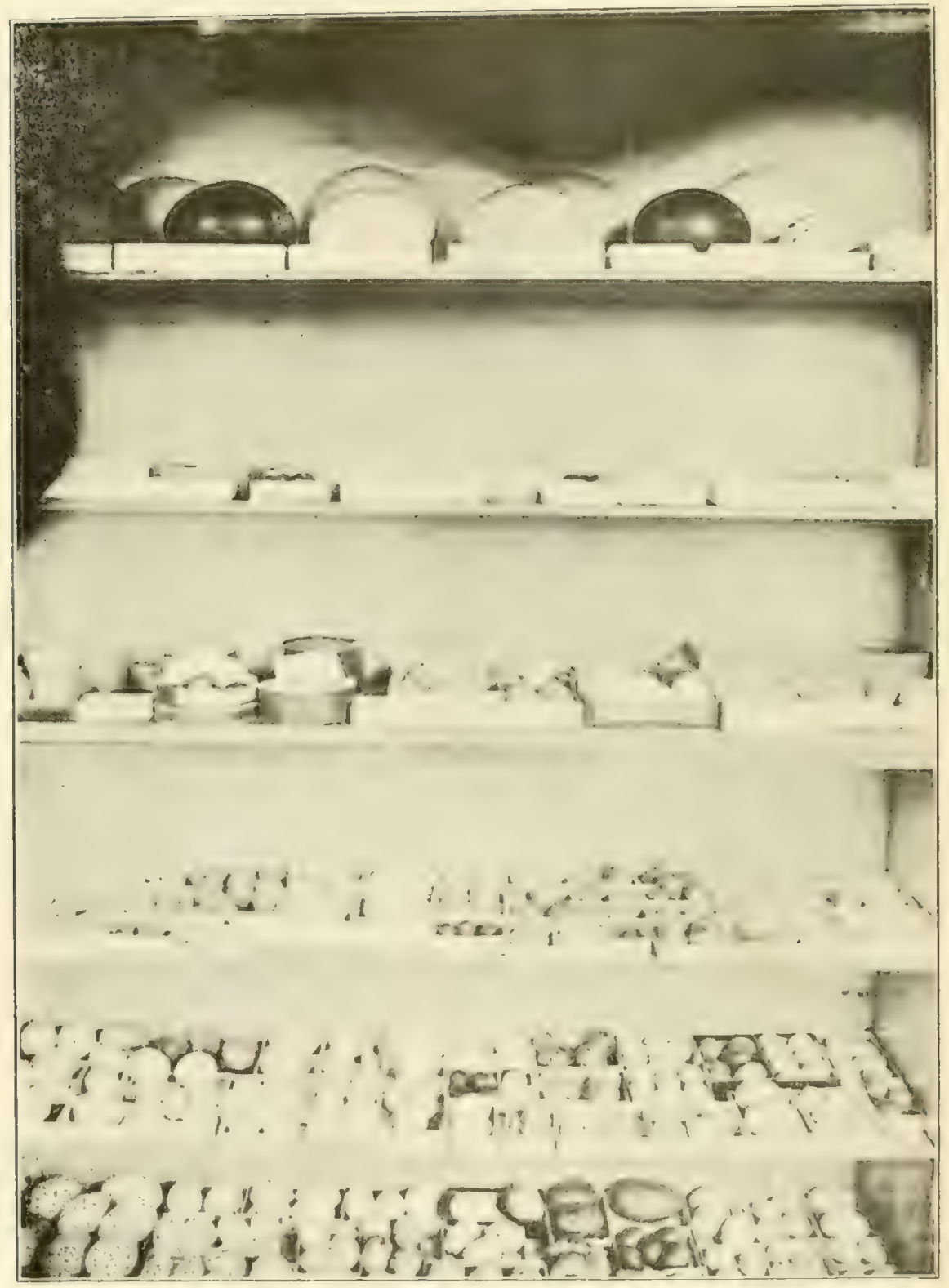

Part of the egg collection of D. Hatch of Oakfield, Wis., showing method of arrangement 


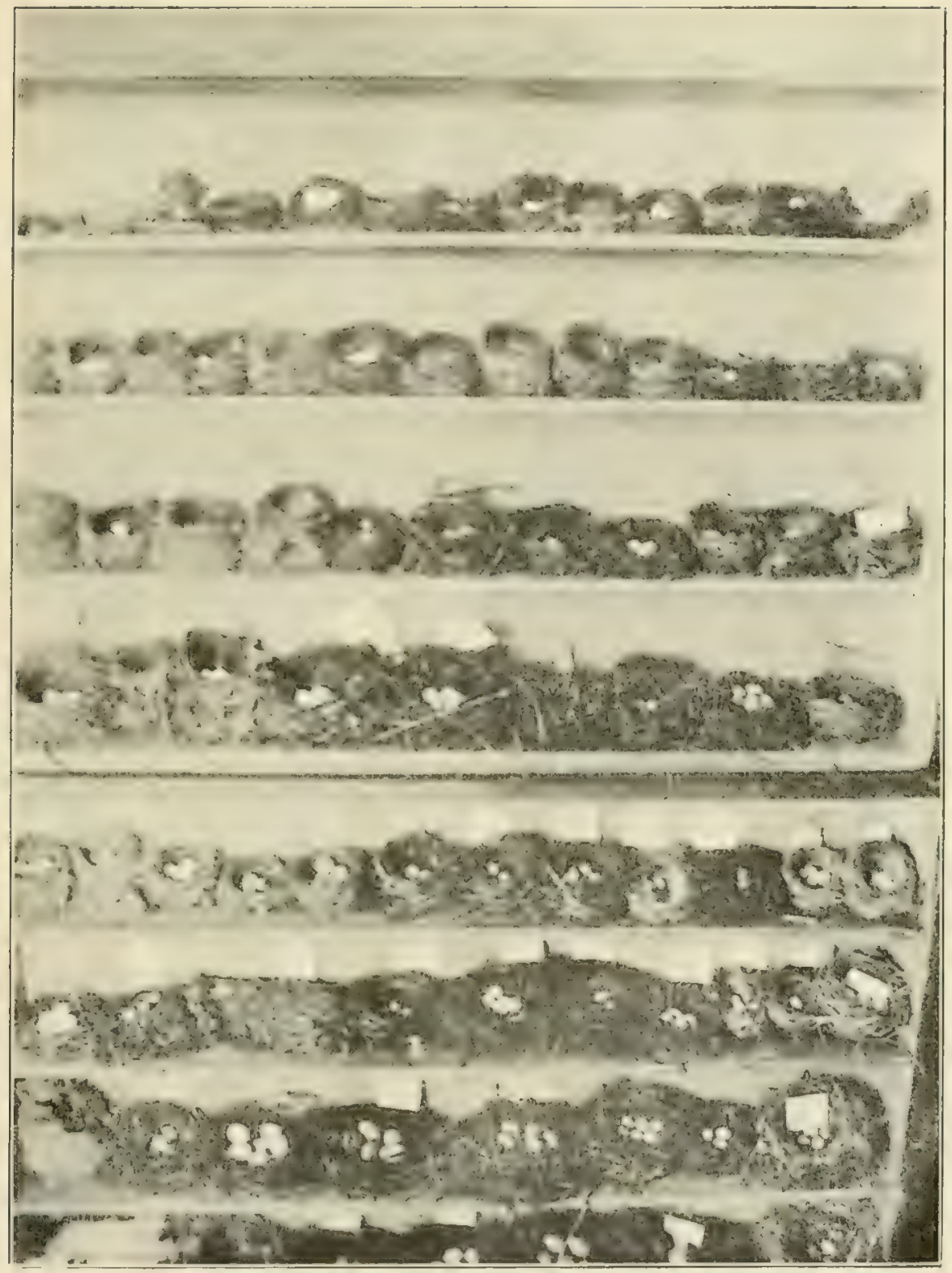

l'art of egrz collection of Delos IIateh of Oakfield, Wis., showing method of arrangenent 


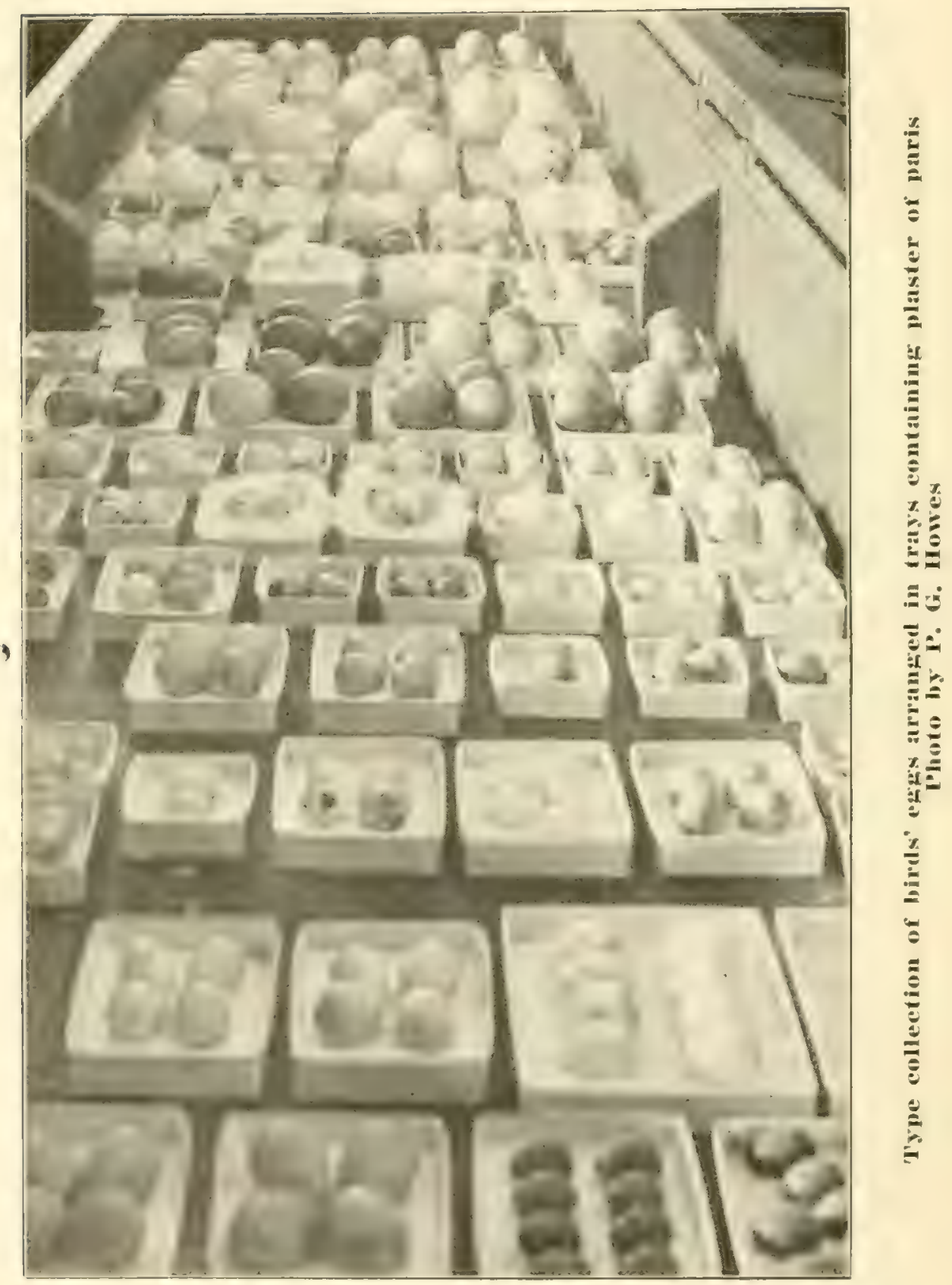




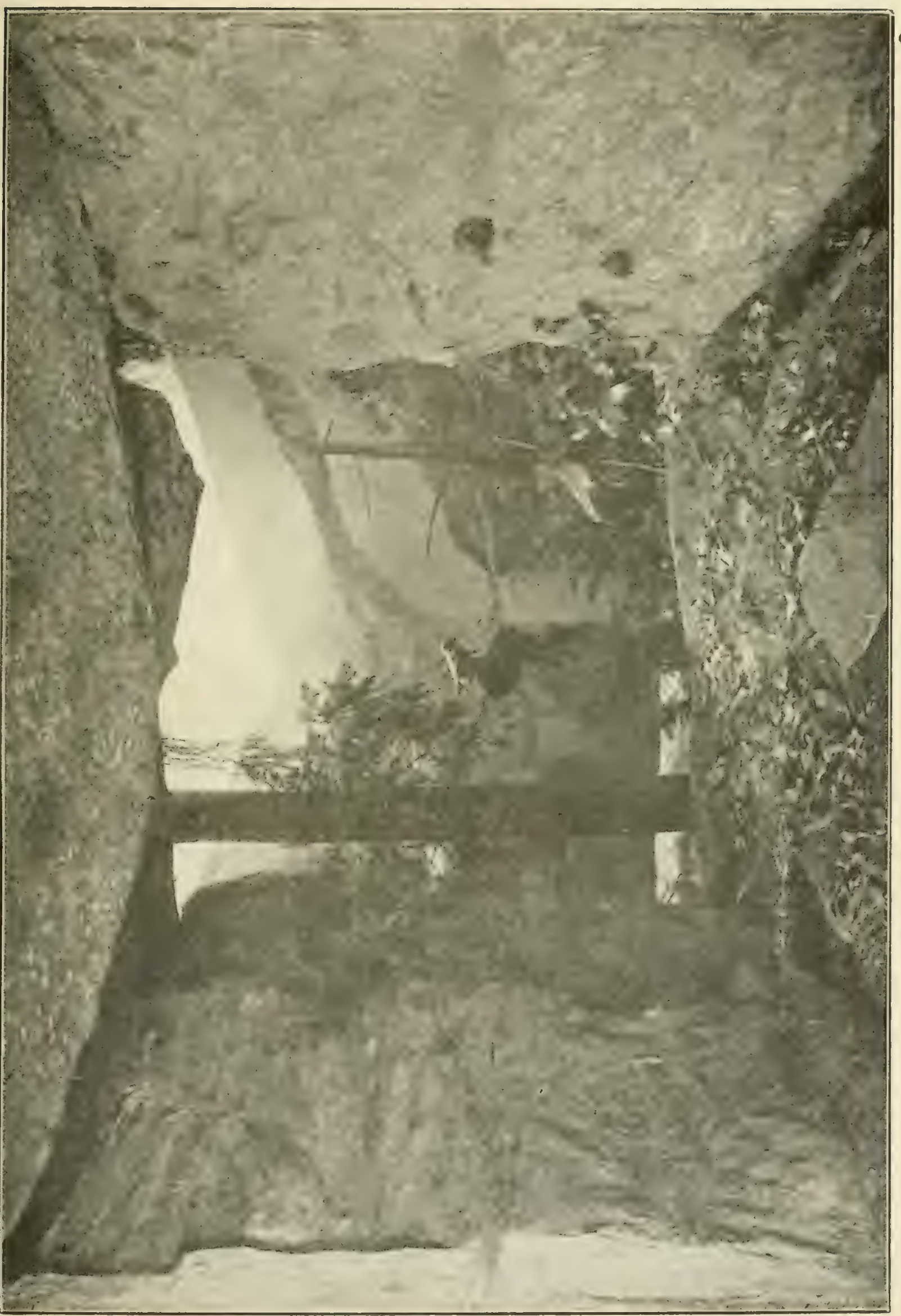

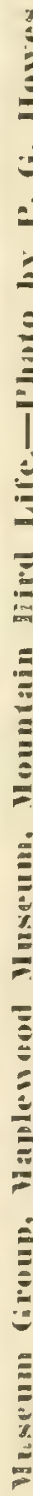




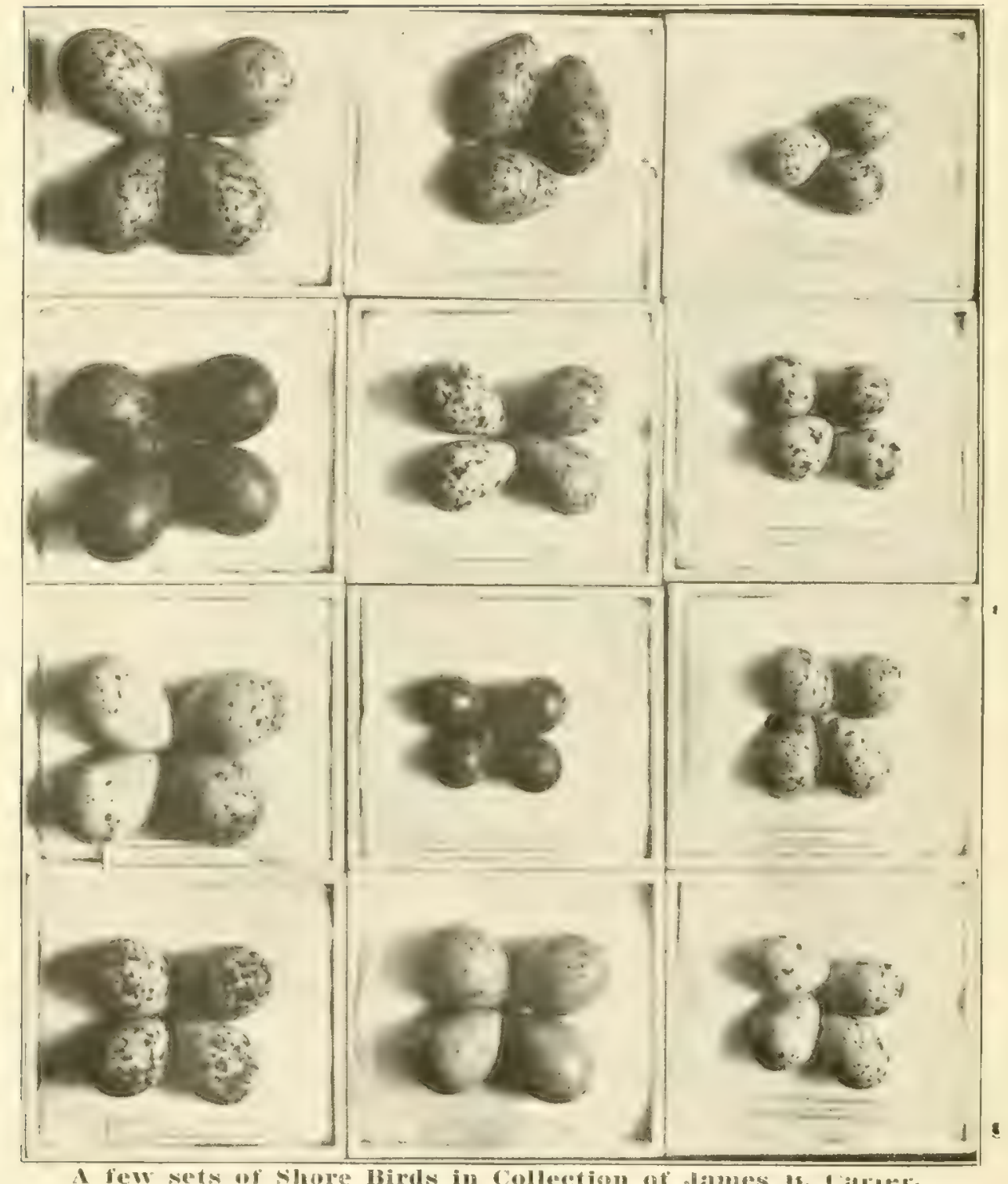

Wayneslourg, På. 


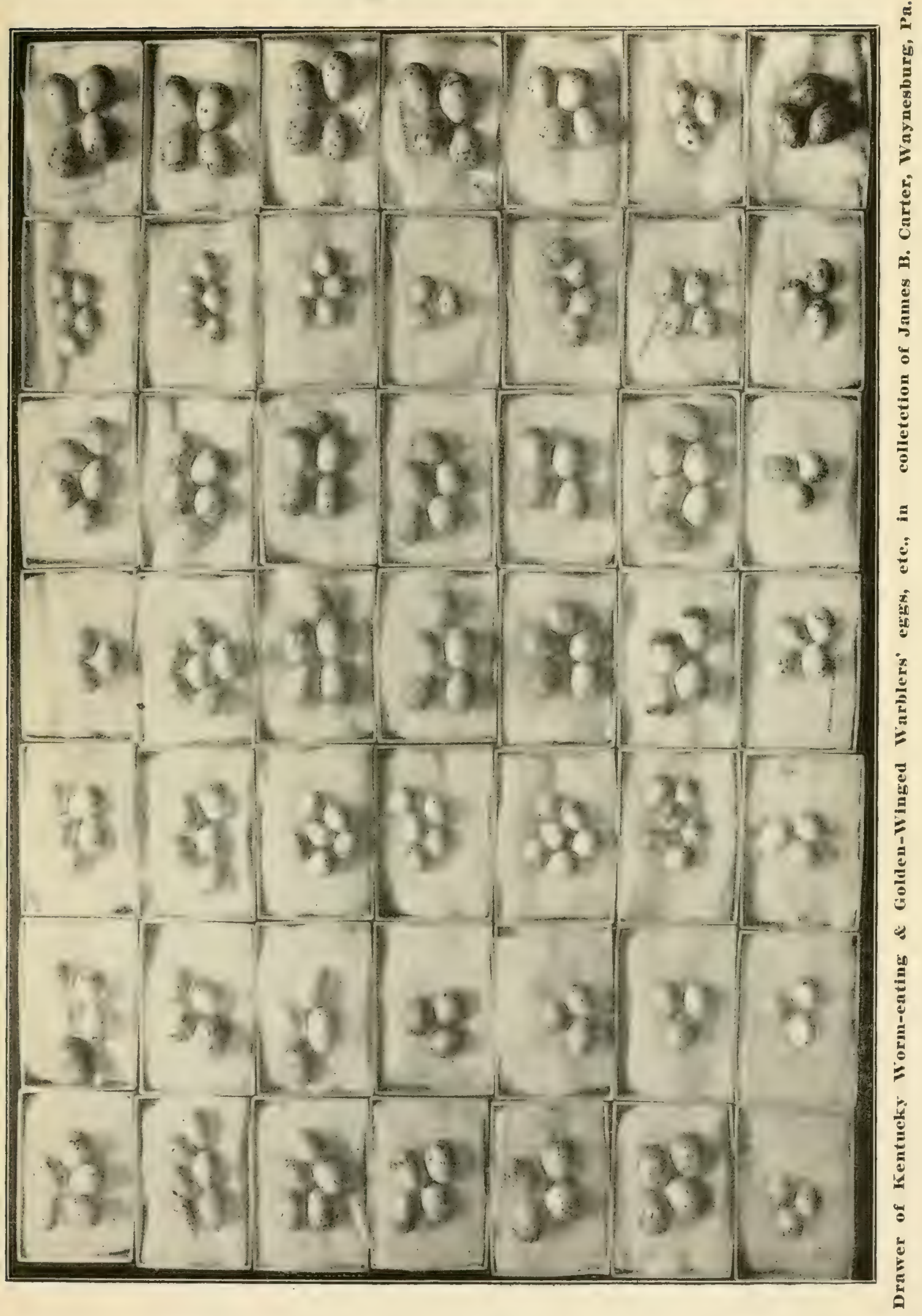




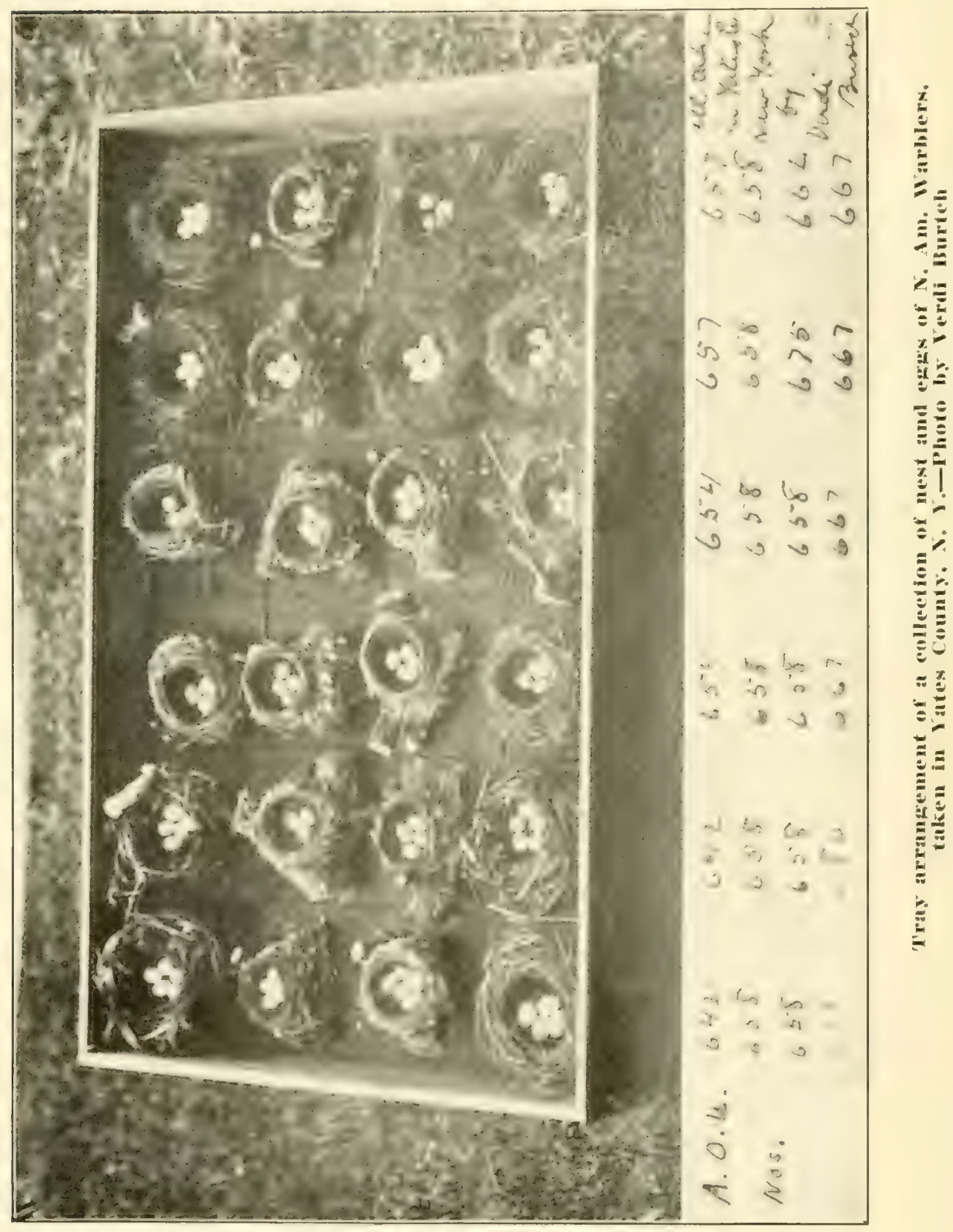




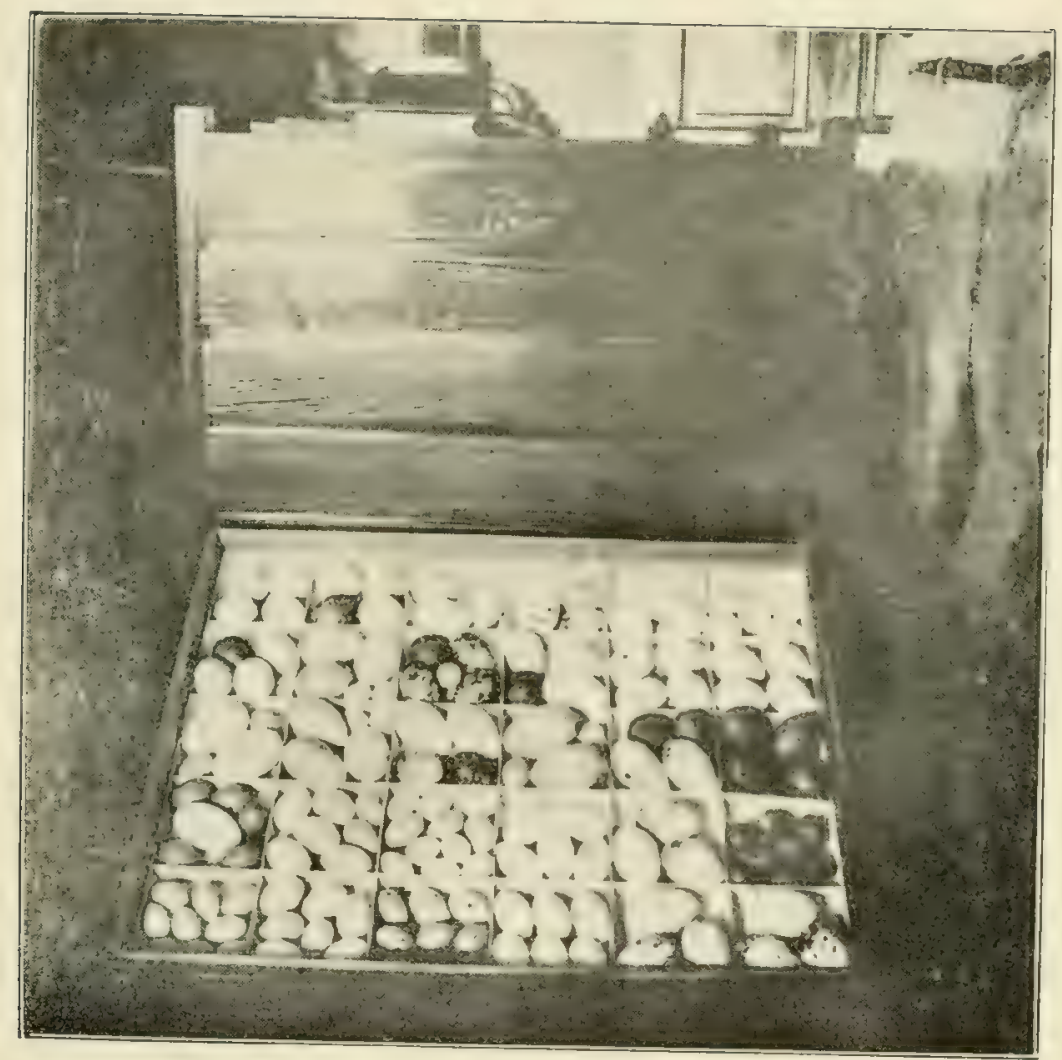

Drawer of Eggs showing arrangements aldopted in the collection of Dr. Perry Photo by rinlay Simmon. 


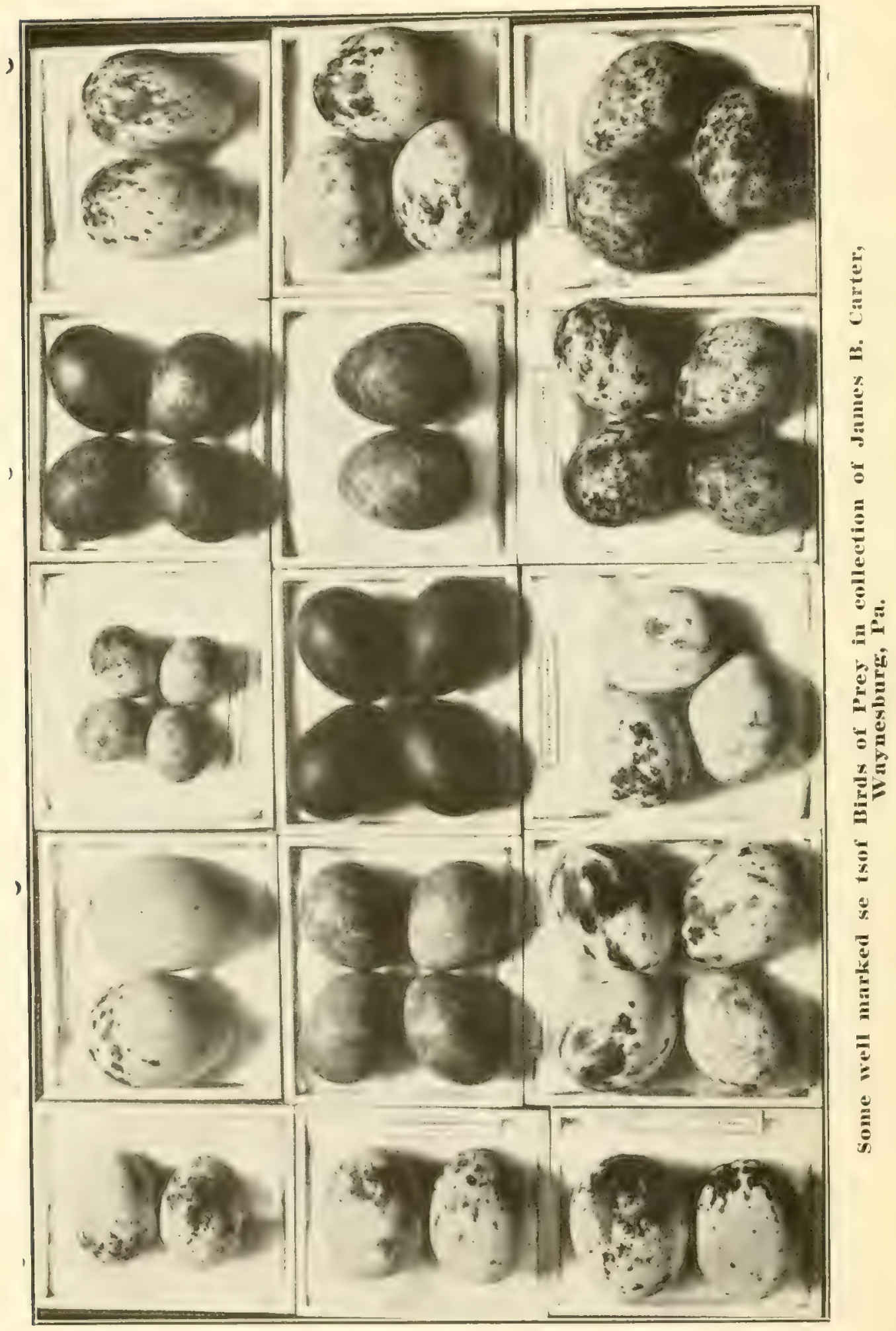




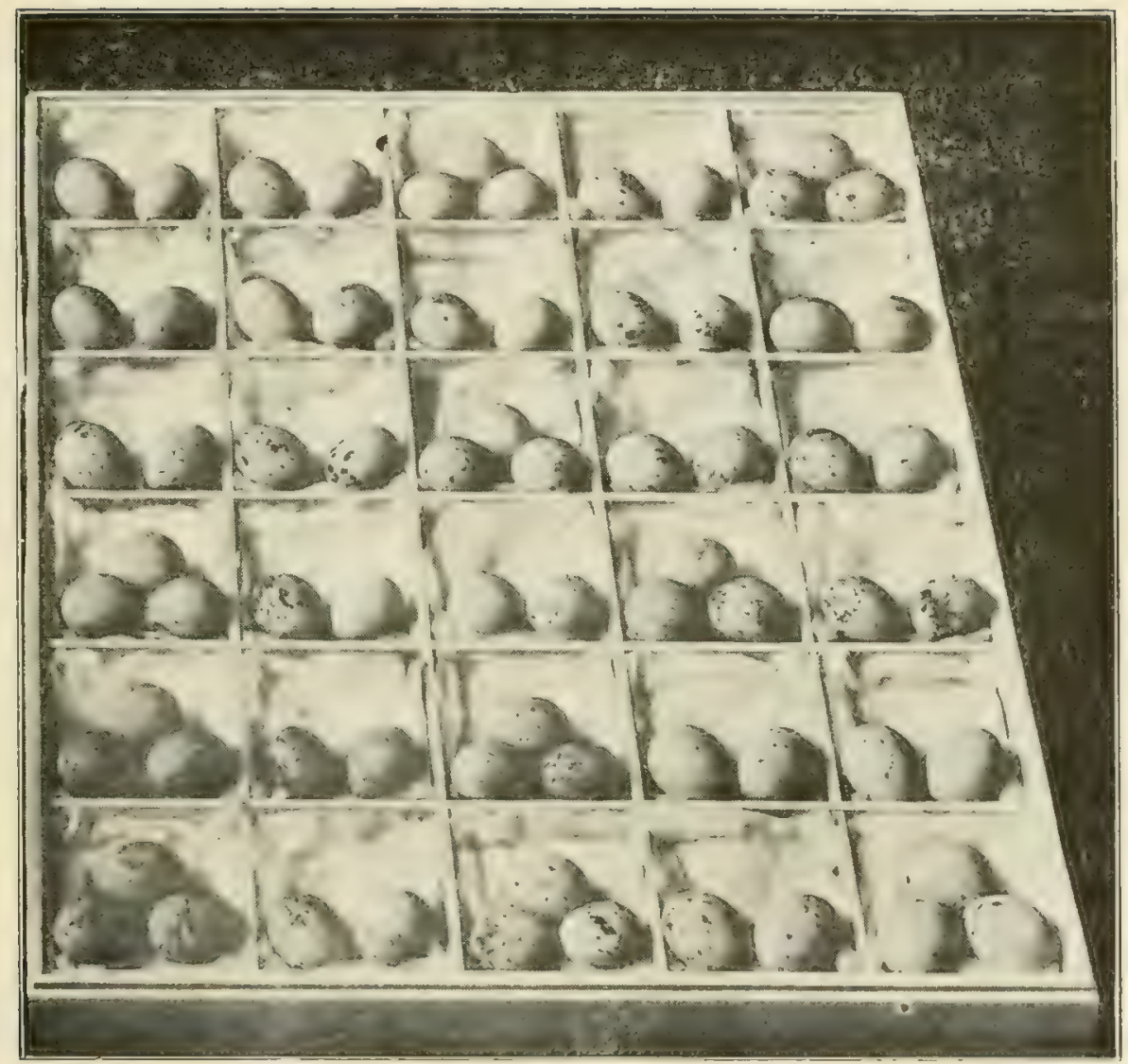

Series of egres of lbell 'lailed liawle's egges in collection of J. Warren Jacolos. IPhoto by J. W. Jacolos 


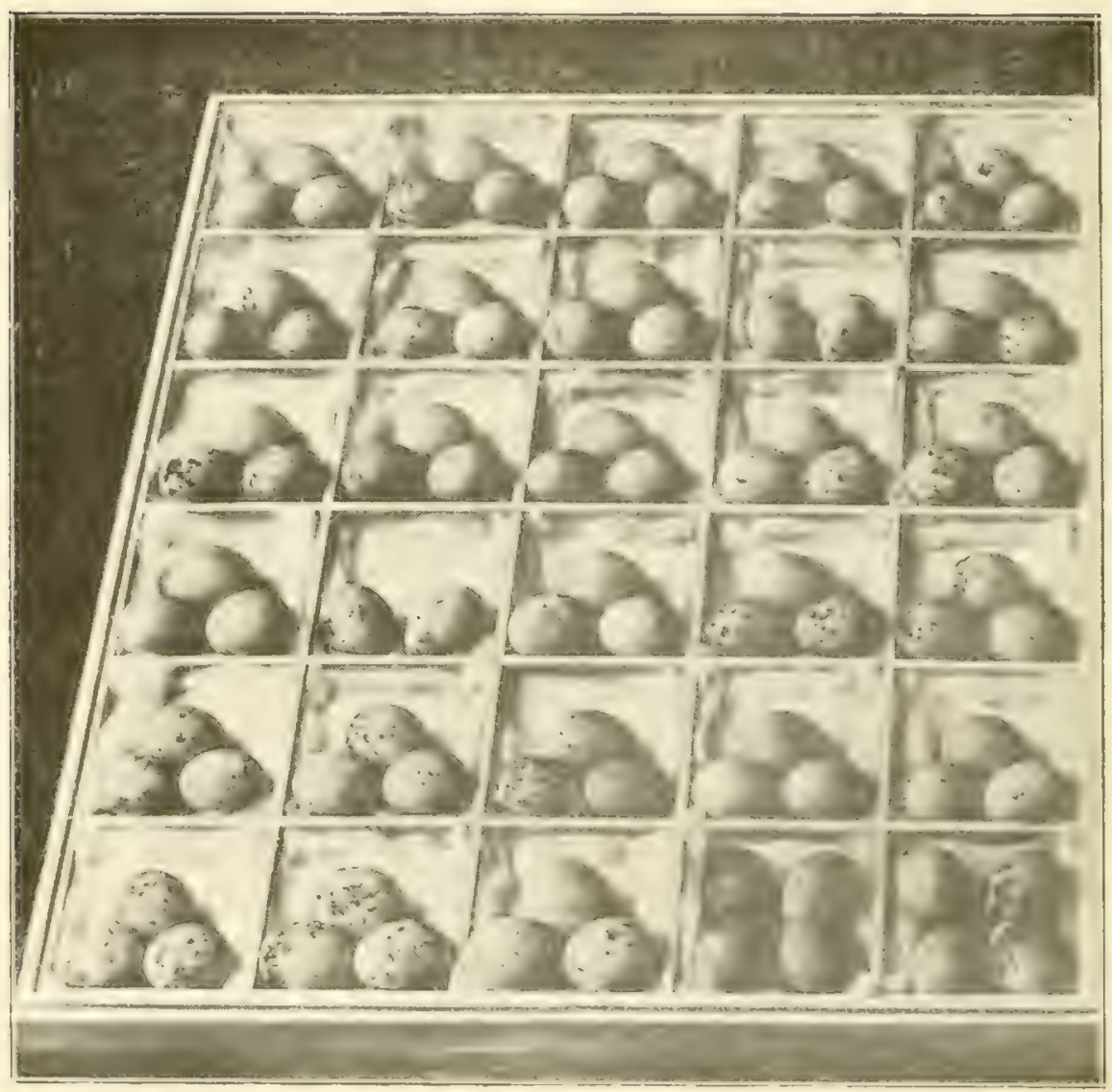

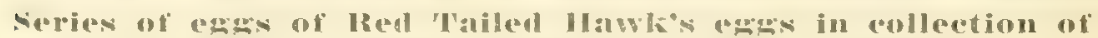

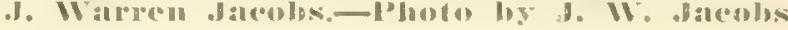




\section{EXCHAHGE PRICE LIST}

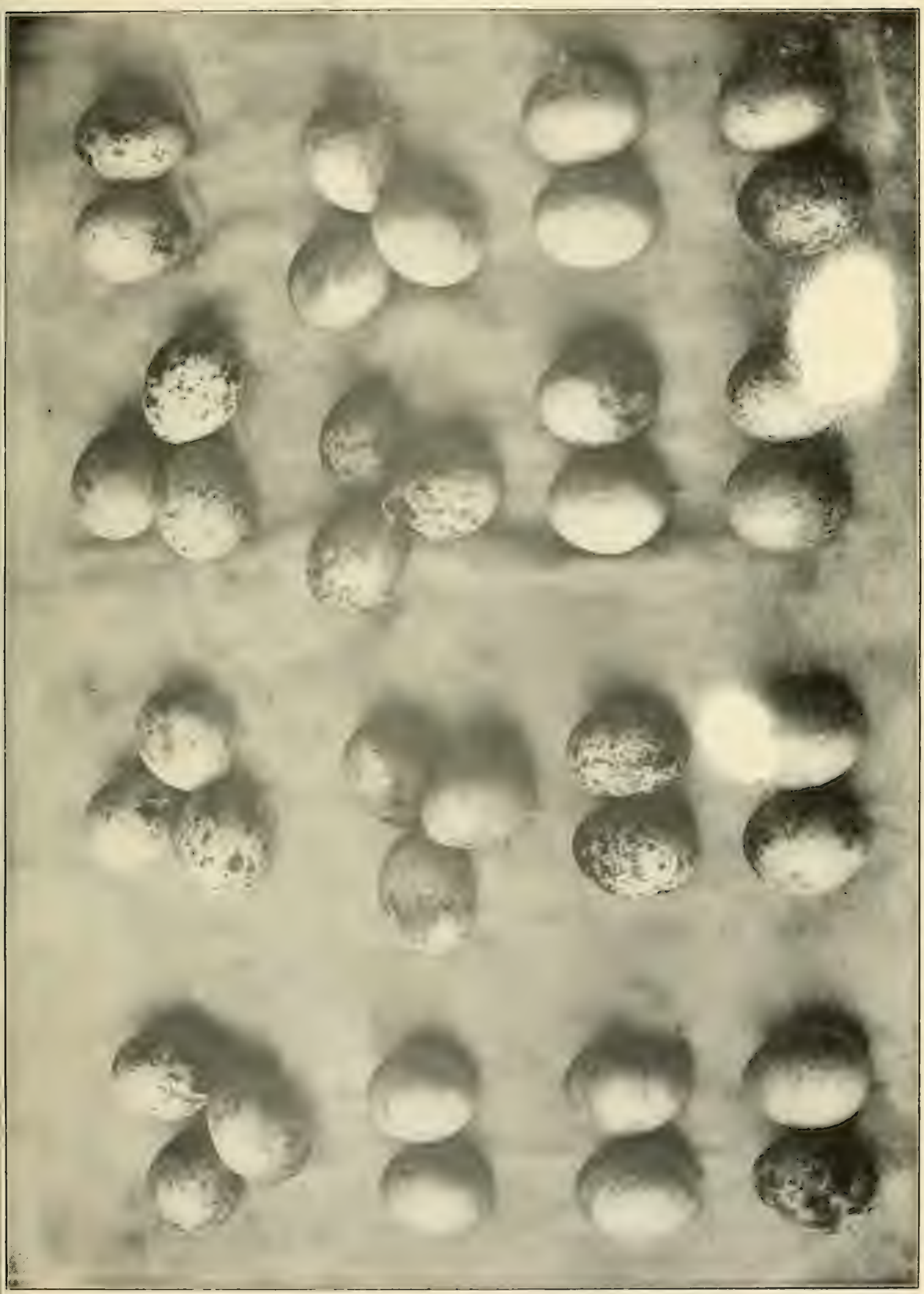

Series of Broad-winged Hawls in collection of J. F. Stierle and Charles Pelton.

Plhoto by J. F'. Stierle Most of this series now rests in the collection of $\mathrm{H}$. M. Harnes 
THE AMERICAN OOLOGISTS'

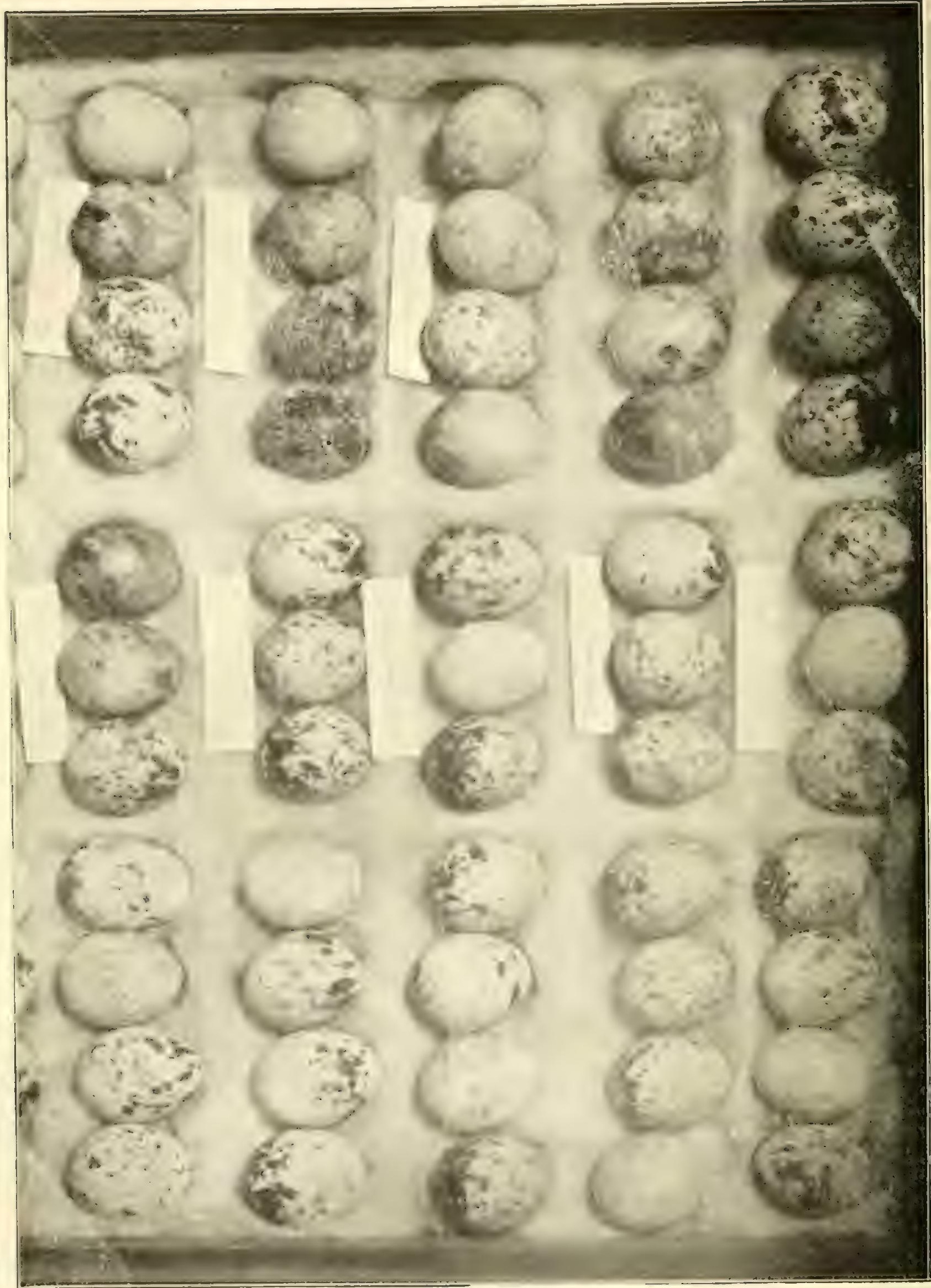




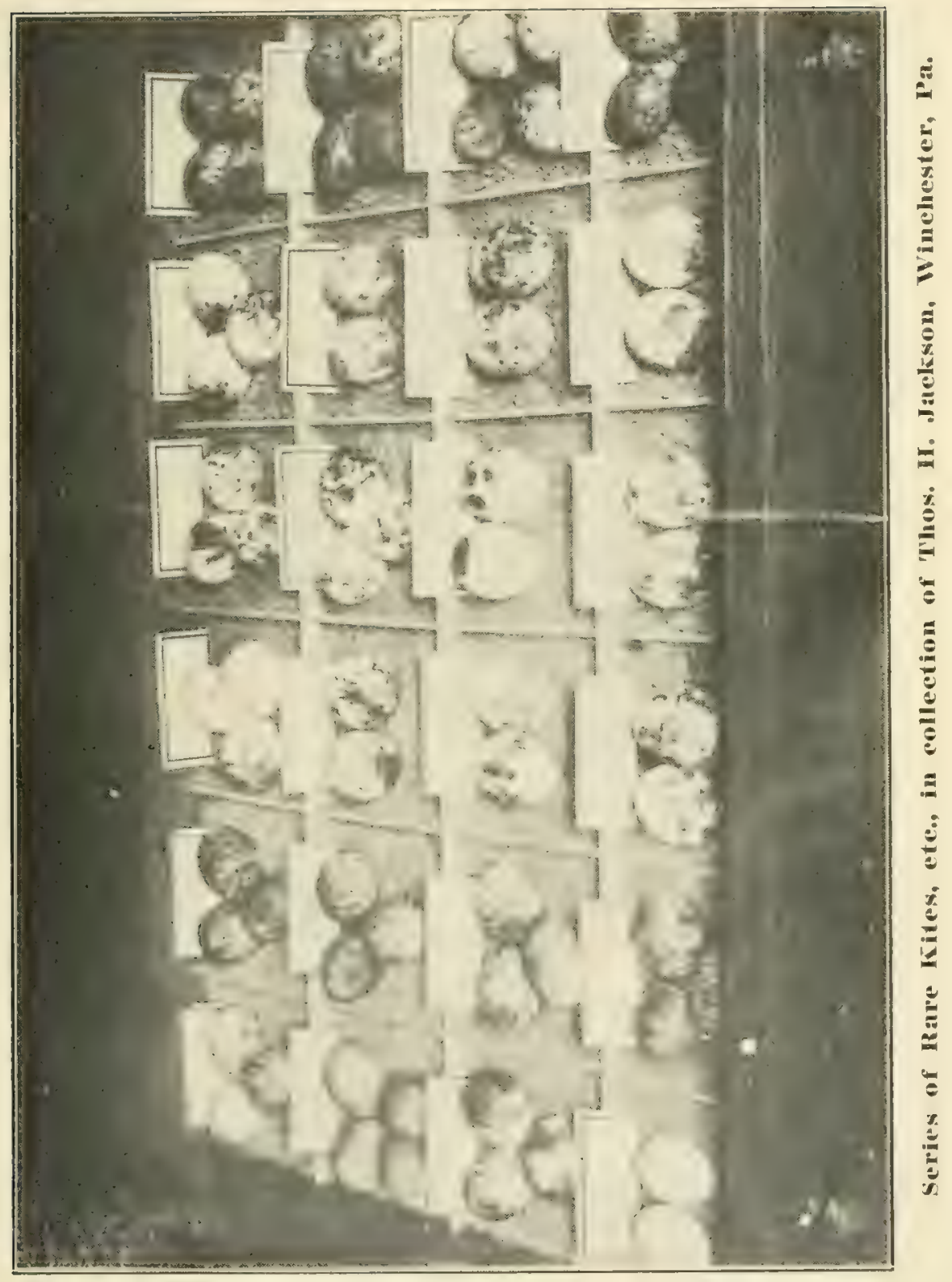




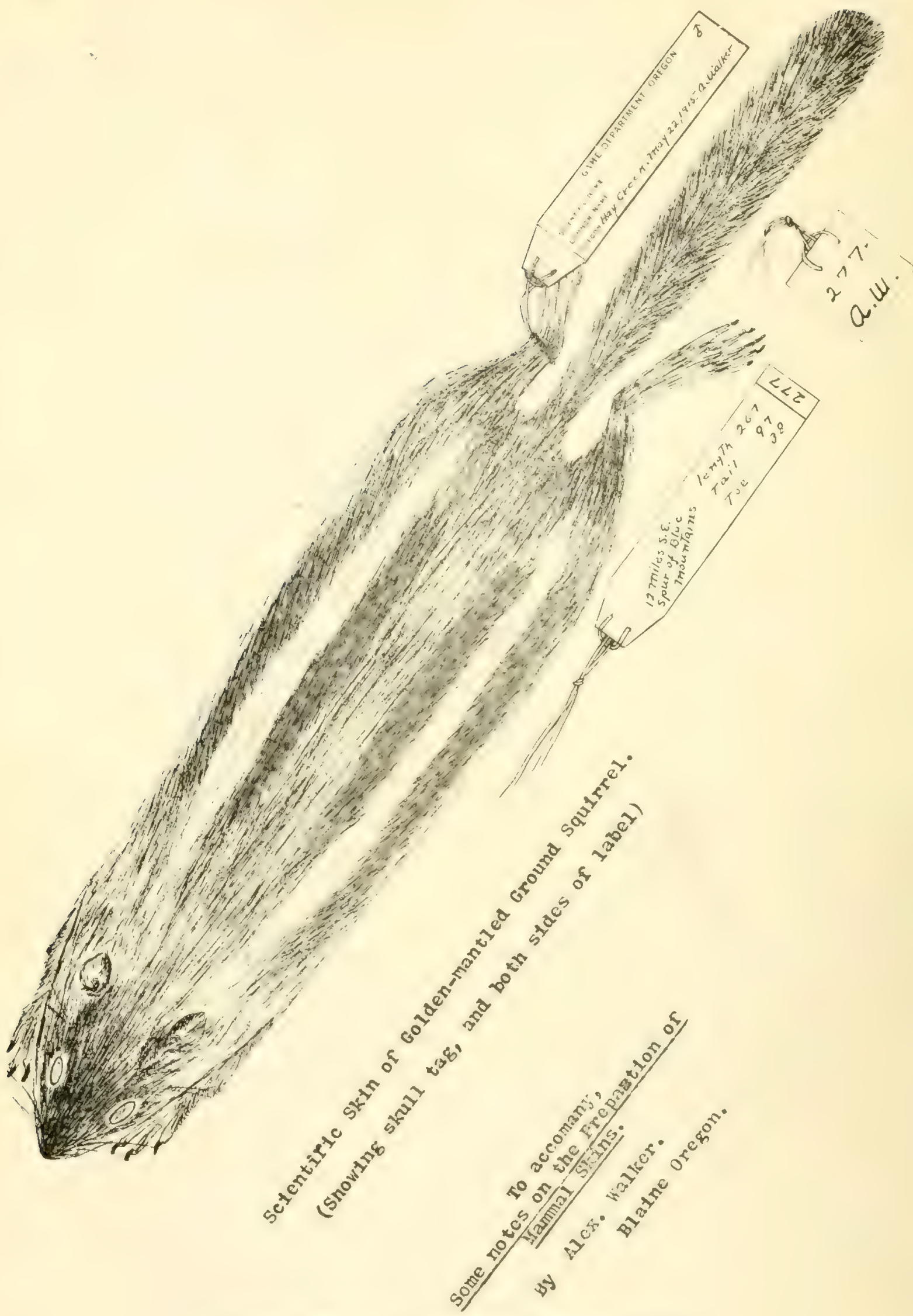

Proper Method of preparing Iammal skins

Pen drawing by Alex Walker, Hlainc, Oregon. 


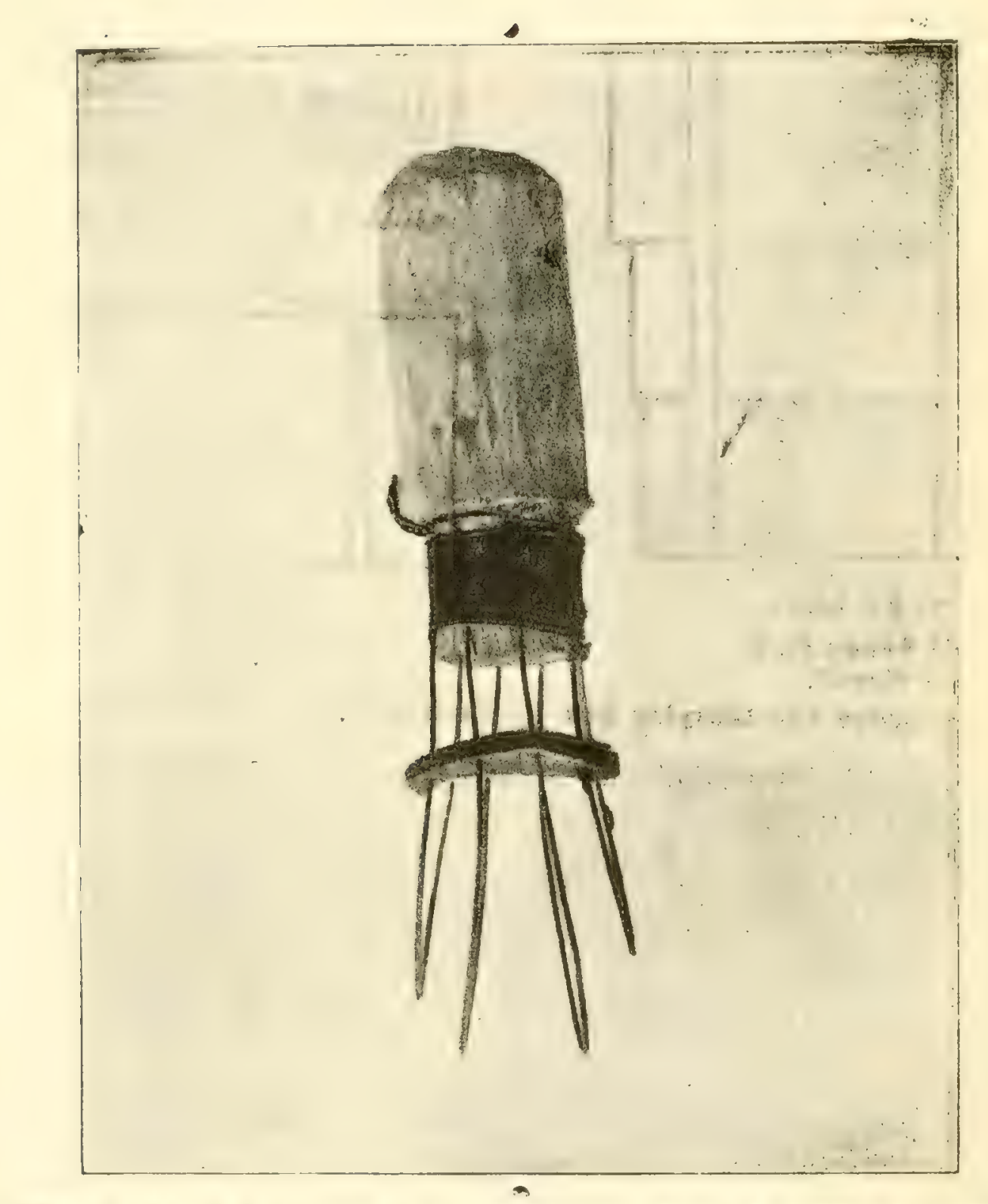

"Egs Grabber" Origing̣ted by Charles Littlejohn of Redvood, Enlurornig 


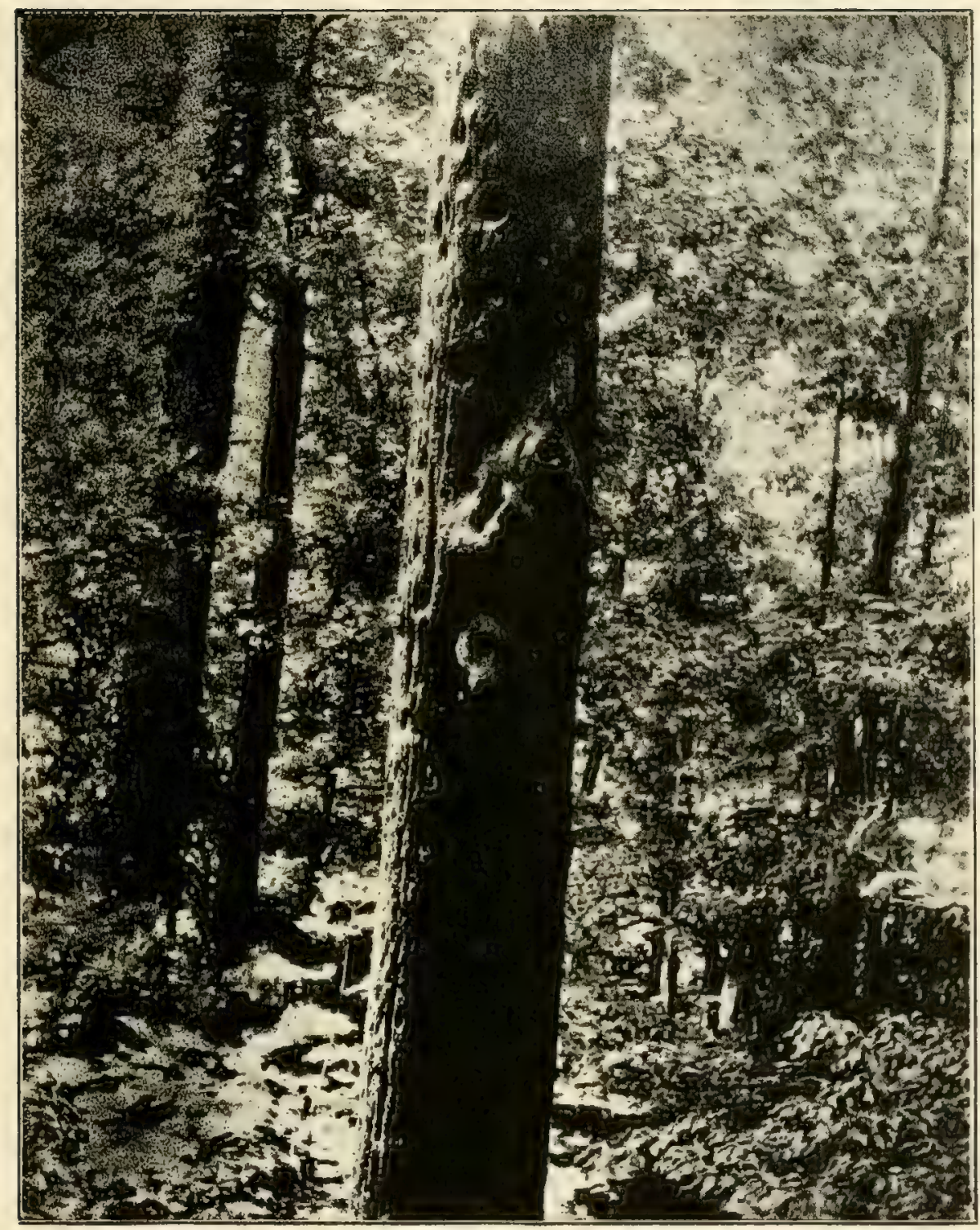

White oak snag near Waynesburg, Pa., showing excavation of Pileated Wood. pecker, (the hole near the top) - Ph oto by S. S. Dickey. 



\section{EXCHANGE PRICE LIST}

OF

\section{North American Birds' Eggs Included in the}

\section{American Ornithologist's Union Check List.}

\section{2}

1. Acchmophorus occidentalis ....\$ .40

Western Grebe

¿ C'olymbus holboelli .......... 5.

Holboll's Grebe

: Colymbus auritus ........... .35

Horned Grebe

4 Colymbus nigricollis californicus $\quad .20$

Eared Grebe

5 Colymbus dominicus brachypter-

us $\ldots \ldots \ldots \ldots \ldots \ldots \ldots \ldots \ldots \ldots . . .95$

Mexican Grebe

if Podilymbus podiceps ... .... .15

Pied-billed Grebe

† Gavia immer ............. . 3.90

Loon

s Gavia adamsi ............ 30.00

Yellow-billed Loon

9 Gavia arctica .................5

Black-throated Loon

10 Gavia pacifica ........... 5.50

Pacific Loon

11 Gavia stellata ............. 3.25

Red-throated Loon $\quad * 1.00$

1. I unda cirrhata ............ $\quad .80$

'Tufted Puffin

13 Fratercula arctica arctica..... 1.25

Puffin $\quad * .50$

1:in Fratercula arctica naumanni... $\quad 6.00$

Iarge-billed Puffin

14 Fratercula corniculata ....... 3.50

Horned Puffin

15) Cerorhinca monocerata ...... 3.50

Rhinoceros Auklet 

16 Ptychoramphus aleuticus .....

Per
Egg

Cassin's Auklet

17 Phaleris psittacula ......... 10.00

Paroquet Auklet

18 Athia cristatella .......... 10.00

Crested Auklet

19 Athia pygmza

Whiskered Auklet

20 AEthia pusilla ............. 4.00

Least Auklet

21 Synthliboramphus antiquus ... $\quad 3.50$

Ancient Murrelet

23 Brachyramphus marmoratus...

Mirbled Murrelet

24 Brachyramphus brevirostris ...

Kittlitz's Murrelet

25 Brachyramphus hypoleucus ....

Xantus's Nurrelet

26 Brachyramphus craverii ...... 8.50

Craveri's Murrelet

27 Cepphus grylle ............. 1.60

Black Guillemot $\quad * .40$

28 Cepphus mandti .......... 4.50

Mandt's Guillemot

29 Cepphus columba .......... 9.90

Pigeon Guillemot

30 Uria troille troille.......... .35

Murre

$30 a$ Uria troille californica....... $\quad .35$

California Murre

31 Uria lomvia lomvia......... $\quad 1.00$

Brünnich's Murre

31 Uria lomvia arra........... 1.00

Pallas's Murre

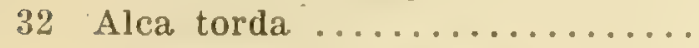

Razor-billed Auk

33 Plautus impennis

Great Ank

34 Alle alle

Dovekie

35 Megalestris skua .......... 2.90

Skua

36 Stercorarius pomarinus ...... $\quad 12.00$

Pomarine Jaeger $\quad * 2.50$

37 Stercorarius parasiticus ...... $\quad .60$

Parasitic Jaeger

38 Stercorarius longicaudus ...... 3.70

Long-tailed Jaeger \& $\quad * 1.50$

39 Pagophila alba ........... 1500

Ivory Gull

40 Rissa tridactyla tridactyla..... $\quad 1.25$

Kittiwake $\quad .40$

40 Rissa tridactyla pollicaris..... 2.00 Pacific Kittiwake 



\begin{tabular}{|c|c|c|}
\hline \multicolumn{2}{|c|}{$\begin{array}{l}1910 \\
\text { A.O.U. } \\
\text { No. }\end{array}$} & $\begin{array}{l}\text { Price } \\
\text { Per } \\
\text { Egg }\end{array}$ \\
\hline 41 & $\begin{array}{l}\text { Rissa brevirostris .......... } \\
\text { Red-legged Kittiwake }\end{array}$ & 4.30 \\
\hline 42 & $\begin{array}{l}\text { Larus hyperboreus .......... } \\
\text { Glaucous Gull }\end{array}$ & 4.10 \\
\hline 43 & $\begin{array}{l}\text { Larus leucopterus ........... } \\
\text { Iceland Gull }\end{array}$ & 4.00 \\
\hline 44 & $\begin{array}{l}\text { Larus glaucescens .............. } \\
\text { Glaucous-winged Gull }\end{array}$ & 1.35 \\
\hline 45 & $\begin{array}{l}\text { Larus kumlieni ............... } \\
\quad \text { Kumlien's Gull }\end{array}$ & \\
\hline 16 & $\begin{array}{l}\text { Larus nelsoni ........................ } \\
\text { Nelson's Gull }\end{array}$ & \\
\hline 17 & $\begin{array}{l}\text { Larus marinus ............... } \\
\text { Great Black-backed Gull }\end{array}$ & 1.65 \\
\hline 48 & $\begin{array}{l}\text { Larus schistisagus ............ } \\
\text { Slaty-backed Gull }\end{array}$ & 4.60 \\
\hline 49 & $\begin{array}{l}\text { Larus occidentalis ........... } \\
\text { IVestern Gull }\end{array}$ & .45 \\
\hline 150$]$ & $\begin{array}{l}\text { Larus affinis . ................ } \\
\quad \text { Siberian Gull }\end{array}$ & 4.10 \\
\hline 51 & $\begin{array}{l}\text { Larus argentatus } \ldots \ldots \ldots \ldots \ldots \\
\quad \text { Herring Gull }\end{array}$ & .40 \\
\hline$[52]$ & 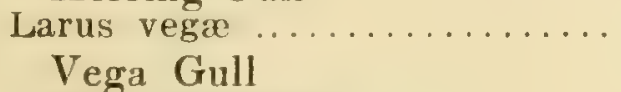 & 5.60 \\
\hline 53 & $\begin{array}{l}\text { Larus californicus ........... } \\
\text { California Gull }\end{array}$ & .45 \\
\hline 54 & $\begin{array}{l}\text { Larus delawarensis .......... } \\
\quad \text { Ring-billed Gull }\end{array}$ & .55 \\
\hline 55 & $\begin{array}{l}\text { Larus brachyrhynchus ......... } \\
\text { Short-billed Gull }\end{array}$ & 6.00 \\
\hline$[56]$ & $\begin{array}{l}\text { Larus canus } \ldots \ldots \ldots \ldots \ldots \ldots \\
\text { Mew Gull }\end{array}$ & .40 \\
\hline 57 & $\begin{array}{l}\text { Larus heermanni ............. } \\
\text { Heermann'ş Gull }\end{array}$ & 8.00 \\
\hline 58 & $\begin{array}{l}\text { Larus atricilla } \ldots \ldots \ldots \ldots \ldots \\
\quad \text { Laughing Gull }\end{array}$ & .30 \\
\hline 59 & $\begin{array}{l}\text { Larus franklini } \ldots \ldots \ldots \ldots \ldots \ldots \\
\text { Franklin's Gull }\end{array}$ & .65 \\
\hline 60 & $\begin{array}{l}\text { Larus philadelphia .......... } \\
\text { Bonaparte's Gull }\end{array}$ & 9.50 \\
\hline$[60.1]$ & $\begin{array}{l}\text { Larus minutus } \ldots \ldots \ldots \ldots \ldots \\
\text { Little Gull }\end{array}$ & 4.00 \\
\hline 61 & $\begin{array}{l}\text { Rhodostethia rosea ........... } \\
\text { Ross's Gull }\end{array}$ & \\
\hline 62 & $\begin{array}{l}\text { Xema sabini } \ldots . . . \ldots \ldots \ldots \ldots \\
\text { Sabine's Gull }\end{array}$ & 7.00 \\
\hline 63 & $\begin{array}{l}\text { Gelochelidon nilotica } . . . . . . \\
\text { Gull-billed Tern }\end{array}$ & .65 \\
\hline 64 & $\begin{array}{l}\text { Sterna caspia } \ldots \ldots \ldots \ldots \ldots \\
\text { Caspian Tern }\end{array}$ & .65 \\
\hline 65 & $\begin{array}{l}\text { Sterna maxima } \ldots \ldots \ldots \ldots \ldots \\
\text { Royal Tern }\end{array}$ & .35 \\
\hline 66 & $\begin{array}{l}\text { Sterna elegans } \ldots \ldots \ldots \ldots \ldots \\
\text { Elcrant Tern }\end{array}$ & 10.00 \\
\hline
\end{tabular}



1910

A.O.U.

No.

Price

Per

67 Sterna sandvicensis acuflavida.. $\quad .50$

Cabot's Tern

[68] Sterna trudeaui

25.00

Trudeau's Tern

69 Sterna forsteri ............

Forster's Tern

70 Sterna hirundo ............. .15

Common Tern

71 Sterna paradisæa .......... $\mathbf{4 5}$

Aretic Tern

7: Sterna dougalli

Roseate 'Tern

73 Sterna aleutica

Aleutian 'Tern

74 Sterna antillarum

$$
\text { Least Tern }
$$

75 Sterna fuscata

\section{Sooty 'Tern}

[76] Sterna anætheta

Bridled Tern

77 Hydrochelidon nigra surinamensis $\ldots \ldots \ldots \ldots \ldots \ldots \ldots \ldots . . .20$

Black Teri.

[78] Hydrochelidon leucoptera ...... $\quad .80$

White-winged Black Tern

79 Anoüs stolidus

Noddy

80 Rynchops nigra

Black Skimmer

81 Diomedea nigripes

Black-footed Albatross

82 Diomedea albatrus

Short-tailed Albatross

82.1 Diomedea, immutabilis

10.50

Laysan Albatross

[83] Thalassogeron culminatus

11.00

Yellow-nosed Albatross

[84] Phœbetria palpebrata

Sooty Albatross

[85] Macronectes giganteus ....... $\mathbf{1 6 . 5 0}$

Giant Fulmar

S6 Fulmarus glacialis glacialis .... $\quad .90$

Fulmar

S6) Fulmarus glacialis glupischa... 3.00

Pacific Fulmar

86.1 iTulmarus rodgersi

Rodgers's Fulmar

87 Priocella glacialoides........ 1550

Slender-billed Fulmar

[10)!] Daption "capense

Pintado Petrel

8S Puffinus borealis

Cory's Shearwater 

\$. Puffinus gravis ............

Greater Shearwater

[90] Puffinus puffinus

Manx Shearwater

91 Puffinus creatopus ......... 10.00

Pink-footed Shearwater

92 Puffinus lherminieri ..........

Audubon's Shearwater

[92.1] Puffinus a'ssimilis ..........

Allied Shearwater

93 Puffinus opisthomelas

Black-vented Shearwater

93.1 Puffinus auricularis

Townsend's Shearwater

95 Puffinus griscous

Sooty Shearwater

96 Puffinus tenuirostris

Slender-billed Shearwater

96.1 Puffinus cuneatus ............

Wedge-tailed. Shearwater

962 Puffinus bulleri

New Zealand Shearwater

[97] Priofinus cinereus

Black-tailed Shearwater

1981 Astrelata hasitata ...........

Black-capped Petrel

99 Astrelata scalaris

18.00

Scaled Petrel

100 Asstrelata fisheri

Fisher's Petrel

[101] Bulweria bulweri

Bulwer's Petrel

103 Halocyptena microsoma ......

Least Petrel

104 Thalassidroma pelagica .......

Storm Petrel

105 Oceanodroma furcata .........

Forked-tailed Petrel

105.2 Oceanodroma kaedingi

Kaeding's Petrel

106 Oceanodroma leucorhoa

Leach's Petrel

106.1 Oceanodroma macrodactyla ...

20.00

Guadalupe Petrel

[106.2] Oceanodroma castro

10.50

Hawaiian Petrel

107 Oceanodroma melania

Black Petrel

108 Oceanodroma homochroa .....

Ashy Petrel

108.1 Oceanodroma socorroensis ....

Socorro Petrel

109 Oceanites oceanicus

Wilson's Petrel 

1910

A.O.U

No.

-[110] Fregetta grallaria

White-bellied Petrel

[111] Pelagodroma marina

White-faced Petrel

112 Phaëthon americanus

Yellow-billed Tropic-bird

113 Phaëthon æthereus ...........

Red-billed Tropic-bird

[113.1] Phaëthon rubricaudus .......

Red-tailed Tropic-bird

[114] Sula cyanops ...............

Blue-faced Booby

114.1 Sula nebouxi

Blue-footed Booby

115 Sula leucogastra

Booby

115.1 Sula brewsteri

Brewster's Booby

[116] Sula piscator

Red-footed Booby

117 Sula bassana

Gannet

118 Anhinga anhinga

Water-Turkey

119 Phalacrocorax carbo

Cormorant

120 Phalacrocorax auritus auritus...

Double-crested Cormorant

120a. Phalacrocorax auritus floridanus

Florida Cormorant

1206 Phalacrocorax auritus cincinatus

White-crested Cormorant

$120 \mathrm{c}$ Phalacrocorax auritus albociliatus .....................

Farallon Cormorant

121 Phalacrocorax vigua mexicanus

Mexican Cormorant

122 Phalacrocorax penicillatus ....

Brandt's Cormorant

123 Phalacrocorax pelagicus pelagi-

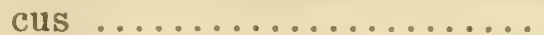

Pelagic Cormorant

$123 a$ Phalacrocorax pelagicus robustus...................

Violet-green Cormorant

$123 b$ Phalacrocorax pelagicus resplen-

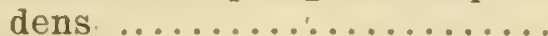

Baird's Cormorant

124 Phalacrocorax urile Red-faced Cormorant

125 Pelecanus erythrorhynchos

White Pelican

126 Pelecanus occidentalis

Brown Pelican
Price

Per

Eigg

10.00

4.00

6.00

10.00

2.50

4.25

6.00

450

.45

$* .40$

.45

2.25

$* .50$

.40

.40

1.20 

1910

A.O.U.

Price

Per

No.

Egg

151 Clangula clangula americana... $\quad 1.00$

Golden-eye

152 Clangula islandica ......... 2.00

Barrow's Golden-eye

153 Charitonetta albeola ........ 8.00

Buffle-head

154 Harelda hyemalis ......... 1.65

Old-squaw *.50

155. Histrionicus histrionicus .... 350

Harlequin Duck $\quad * 1.00$

156 Camptorliynchus labradorius ...

Iabrador Duck

157 Polysticta stelleri

Steller's Eider

158 Arctonetta fischeri

Spectracled Eider

159 Somateria mollissima borealis..

Nortliern Eider

160 Somateria dresseri

$$
\text { Eider }
$$

161 Somateria v-nigra ........ 2.00

Pacific Eider

162 Somateria spectabilis ...... 3.75

King Eider

163 Oidemia americana ........ 2.35

Scoter

[164] Oidemia fusca

Velvet Scoter

165 Oidemia deglandi .......... 2.50

White-winged Scoter

166 Oidemia perspicillata ....... $\mathbf{5 . 0 0}$

Surf Scoter

167 Erismatura jamaicensis ...... $\quad .60$ Ruddy Duck

168 Nomonyx dominicus .........

Masked Duck

169 Chen hyperboreus hyperboreus. 12.00 Snow Goose

$169 a$ Chen hyperboreus nivalis......

Greater Snow Goose

169.1 Chen cærulescens ..........

Blue Goose

170 Chen rossi

Ross's Goose

[171] Anser albifrons albifrons......

European White-fronted Goose

171 $九$ Anser albifrons gambeli...... 400

White-fronted Goose

[171.1] Anser fabalis ........... 1.60

Bean Groose

[171.2] Anser brachyrhynchus...... 3.25

Pink-footed Goose

172 Branta canadensis canadensis.. 2.00 Canada Goose 

$172 a$ Branta canadensis hutchinsi... $\quad \mathbf{4 . 5 0}$

Hutchins's Goose

$172 h$ Branta canadensis occidentalis..

White-cheeked Goose

172 Branta' canadensis minima.. . . .

Cackling Goose

$173 a$ Branta bernicla glaucogastra... Brant

174 Branta nigricans

Black Brant

175 Branta leucopsis

Barnacle Goose

176 Philacte canagica

10.50

Emperor Goose

177 Dendrocygna autunralis ......

Black-bellied Tree-duck

178 Dendrocygna bicolor

Fulvous Tree-duck

[179] Olor cygnus

Whooper Swan

180 Olor columbianus

12.00

Whistling Swan

181 Olor buccinator

Trumpeter Swan

182 Phœnicopterus ruber

300

Flamingo

183 Ajaia ajaja

6.00

Roseate Spoonbill

184 Guara alba

White Ibis

[185] Guara rubra

Scarlet Ibis

186 Plegadis autumnalis

$\because .00$

Glossy Ibis

187 Plegadis guarauna

White-faced Glossy Ibis

188 Mycteria americana

Wood Ibis

[189] Jabiru mycteria

Jabiru

190 Botaurus lentiginosus

Bittern

191 Ixobrychus exilis

\section{Least Bittern}

191.1 Ixobrychus neoxenus

Cory's Least Bittern

192 Ardea occidentalis

Great White Heron

194 Ardea herodias herodias........

Great Blue Heron

194" Ardea herodias fannini. Northwestern Coast Heron

194h Ardea herodias wardi.........

Ward's Heron 

[195] Ardea cinerea

European Heron

196 Herodias egretta ........... 1.50

Egret

197 Egretta' candidissima candidissima .............. 1.00

Snowy Egret

197" Egretta candidissima brewsteri. $\quad \mathbf{5 . 0 0}$ Brewster's Egret

198 Dichromanassa rufescens ..... $\quad .75$

Reddish Egret

199 Hydranassa tricolor ruficollis.. $\quad .25$

L.ouisiana Heron

200 Florida cærulea ........... .25

Little Blue Heron

201 Butorides virescens virescens. . 20

Green Heron

201" Butorides virescens frazari.... $\quad 3.00$

Frazar's Green Heron

201e Butorides virescens anthonyi...

Anthonv's Green Heron

202 Nycticorax nycticorax nævius..

Blnck-crowned Night Heron

203 Nyctanassa violacea ......... .50

Yellow-crowned Night Heron

204 Grus americana ........... 45.00

Whooping Crane

205 Grus canadensis ........... 20.00

Little Brown Crane

206 Grus mexicana .............. 12.00

Sandhill Crane

207 Aramus vociferus ......... 5.00

Limpkin

208 Rallus elegans ............ 40

King Rail

209 Rallus beldingi ............ 20.00

Belding's Rail

210 Rallus obsoletus ........... 100

California Clapner Rail

210.1 Rallus levipes ........... 1.00

Light-footed Rail

211 Rallus crepitans crepitans..... $\quad .35$

Clapper Rail

211" Rallus crepitans saturatus.... $\quad 1.50$

L.ouisiana Clapper Rail

211h Rallus crepitans scotti....... 2.50

Florida Clanner Rail

211e Rallus crepitans waynei...... $\quad .50$

Wayne's Clapner Rail

211.2 Rallus longirostris caribæus... Caribbean Clapper Rail

212 Rallus virginianus ........... 

[213] Porzana porzana

Spotted Crake

214 Porzana carolina ........... .15

Sora

215 Coturnicops noveboracensis ... $\quad 17.00$ Yellow Rail

216 ('recisus jamaicensis

Black Rail

216.1 Creciscus coturniculus ....... 10.00

Farallon Rail

[217] (rex crex ............. .60

Corn Crake

218 Jonornis martinicus ........ $\quad .90$

219 Gallinula galeata ........... .25

Florida Gallinule

[2:0\} Fulica atra ............... $\quad .30$

European Coot

221 Fulica americana .......... .15

Coot

222 Plalaropus fulicarius ....... 5.00

Red Phalarope

223 Lobipes lobatus ... . . . . . . 4.00

Northern Phalarope $\$ 2.50$

224 Steganopus tricolor ......... 1.24

Wilson's Phalarope

225 Recurvirostra americana ..... .50

Avocet

226 Himantopus mexicanus ...... $\quad .40$

Black-necked. Stilt

[227] Scolopax rusticola ........ 4.00

European Woodcock

228 Philohela minor .......... 5.00

Woodcock

[229] Gallinago gallinago ........ . 40

European Snipe

230 Gallinago delicata ......... 3.25

Wilson's Snipe

[230.1] Gallinago media ........ 5.50

Great Snipe

231 Macrorhamphus griseus griseus Dowitcher

232 Macrorhamphu's griseus scolopaceus ................. 10.00

Long-billed Dowitcher

233 Micropalama himantopus....... Stilt Sandpiper

234 Tringa canutus..............

Knot

235 Arquatella maritima maritima.. $\quad \dot{0.50}$

Purple Sandpiper

$235 a$ Arquatella maritima couesi.... 1250

Aleutian Sandpiper 

235bArquatella maritima ptilocnemis.

Pribilof Sandpiper

238 Pisobia aurita.

Sharp-tailed Sandpiper

239 Pisobia maculata ........... Pectoral Sandpiper

240 Pisobia fuscicollis

White-rumped Sandpiper

241 Pisobia bairdi.............. Baird's Sandpiper

242 Pisobia minutilla ... Least Sandpiper

[242.1] Pisobia damacensis . . ....... Long-toed Stint

[243] Pelidna alpina alpina........ Dunlin

243a Pelidna alpina sakhalina Red-backed Sandpiper

244 Erolia ferruginea. Curlew Sandpiper

[245] Eurynorhynchus pygmeus .... Spoon-bill Sandpiper

246 Ereunetes pusillus Semipalmated Sandpiper

247 Ereunetes mauri ........... Western Sandpiper

248 Calidris leucophæa Sanderling

249 Limosa fedoa Marbled Godwit

250 Limosa lapponica baueri... Pacific Godwit

251 Limosa hæmastica Hudsonian Godwit

[252] Limosa limosa ..... Black-tailed Godwit

[253] Glottis nebularia Green-shank

254 Totanus melanoleucus Greater Yellow-legs

255 Totanus flavipes Yellow-legs

256 Helodromas solitarius solitarius Solitary Sandpiper

$256 n$ Helodromas solitarius cinnamoneus ................

IVestern Solitary Sandpiper

[257] Helodromas ocrophus ....... Green Sandpiper

[257.1] Rhyacophilus glareola ...... Wood Sandpiper

258 Catoptrophorus - semipalmatus

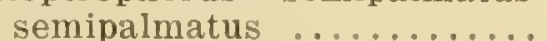
Willet 

$258 a$ Catoptrophorus semipalmatus in-

Egs ornatus ................. 1.50

Western Willet

259 Heteractitis incanus ........

Wandering Tattler

[260] Machetes pugnax .......... $\quad .75$

Ruff

261 Bartramia longicauda ....... $\quad 3.00$

Upland Plover

262 Tryngites subruficollis ........ 15.00

Buff-breasted Sandpiper

263 Actitis macularia .......... .20

Spotted Sandpiper

264 Numenius americanus ....... $\quad 5.00$

Long-billed Curlew

265 Numenius hudsonicus ........ 15.00

Hudsonian Curlew

266 Numenius borealis

Eskimo Curlew

[267] Numenius phæopus

Whimbrel

268. Numenius tahitiensis.

Bristle-thighed Curlew

269 Vanellưs vanellus ......... .25

Lapwing

[269.1] Eudromias morinellus ...... 5.50

Dotterel

270 Squatarola squatarola ....... $\mathbf{1 6 . 5 0}$

Black-bellied Plover

[271] Charadrius apricarius ...... $\quad .60$

European Golden Plover

272 Charadrius dominicus dominicus 13.00

Golden Plover

$272 n$ Charadrius dominicus fulvus... 11.00

Pacific Golden Plover

273 Oxyechus vociferus ........ $\quad .30$

Killdeer

274 Egialitis semipalmata ....... $\quad 3.00$

Semipalmated Plover

275 Egialitis hiaticula ..........40

Ringed Plover

[276] Egialitis dubia ........... so

Little Ringed Plover

277 Agialitis meloda ............ 2.00

Piping Plover

278 Egialitis nivosa

Snowy Plover

[279] Agialitis mongola ......... 8.00

Mongolian Plover

280 Ochthodromus wilsonius ...... .75

Wilson's Plover

281 Podasocys montanus

Mountain Plover 

No.

Per

$\simeq 82$ Aphriza virgata ............

Surf-bird

283 Arenaria interpres interpres....

6.00

Turnstone

$283 a$ Arenaria interpres morinella...

15.00

Ruddy Turnstone

284 Arenaria melanocephala....... 15.00

Black Turnstone

[285] Hæmatopus ostralegus .......

European Oyster-catcher

286 Hæmatopus palliatus..........

Oyster-catcher

286.1 Hæmatopus frazari ..........

Frazar's Oyster-catcher

287 Hæmatopus bachmani .......

Black Oyster-catcher

¿S8 Ja'cana spinosa

Mexican Jacana

289 Colinus virginianus virginianus.

Bob-white

$289 a$ Colinus virginianus floridanus..

Florida Bob-white

2896 Colinus virginianus texanus....

Texas Bob-white

291 Colinus ridgwayi

Masked Bob-white

292 Oreortyx picta picta...........

Mountain Quail

$292 a$ Oreortyx picta plumifera.......

Plumed Quail

$292 b$ Oreortyx picta confinis........

San Pedro Quail

293 Callipepla squamata squamata'.

Scaled Quail

293n. Callipepia squamata castanogas.

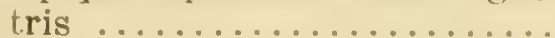

Chestnut-bellied Scaled Quail

294 Lophortyx californica californica California Quail

294a Lophortyx californica vallicola. Valley Quail

295 Lophortyx gambeli Gambel's Quail

296 Cyrtonyx montezumæ mearnsi.. Mearns' Quail

297 Dendragapus obscurus obscurus Dusky Grouse

297 ॥ Dendragapus obscurus fuligino-

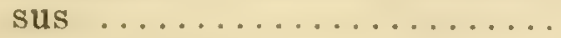

Sooty Grouse

297b Dendragapus obscurus richard-

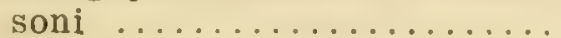

Richardson's Grouse 

1910

A.O.U.

No.

Price

Per

Dendragapus obscurus sierrae.

\section{Sierra Grouse}

298 Canachites canadensis canaden-

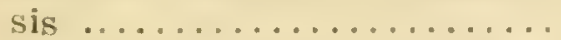

Hudsonian Spruce Partridge

¿981 Canachites canadensis osgoodi..

Alaska Spruce Partridge

2.98c (anachites canadensis canace.. 9.50)

Canadil Spruce Partridge

299 Canachites franklini .........

liranklin's Grouse

:00 Bonasa umbellus umbellus.....

Ruffed Grouse

$: 00 "$ Bonasa umbellus togata.......

Canadil Ruffed Grouse

3006 Bonasa umbellus umbelloides...

Gray Rufted Grouse

300 Bonasa umbellus sabini

\section{Oregon Ruffed Grouse}

:01 Lagopus lagopus lagopus......

IVillow Ptarmigan

301" Lagopus lagopus alleni........

Allen's Ptarmigan

3016 Lagopus lagopus alexandræ....

Alexander's Ptarmigan

302 La'gopus rupestris rupestris.... Rock Ptarmigan

302" Lagopus rupestris reinhardi...

Reinhardt's Ptarmigan

$302 b$ Lagopus rupestris nelsoni

Nelson's Ptarmigan

302r Lagopus rupestris atkhensis....

'Turner's Ptarmigan

302 Lagopus rupestris townsendi...

Townsend's Ptarmigan

$302 e$ Lagopus rupestris chamberlaini. Adak Ptarmigan

$302 f$ Lagopus rupestris dixoni...... Dixon's Ptarmigan

302.1 Lagopus evermanni Evermann's Ptarmigan

303 Lagopus welchi............. 12.00 Welch's Ptarmigan

304 Lagopus leucurus leucurus.... 17.50

White-tailed Ptarmigan

$304 \pi$, Lagopus leucurus peninsularis. 17.50

Kenai White-tailed Ptarmigan

305 Tympanuchus americanus ameri-

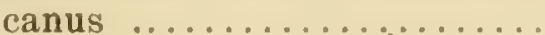

Prairie Chicken

305" Tympanuchus americanus att-

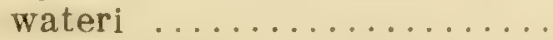



306 Tympanuchus cupido ....... 50.00 Heath Hen

307 Tympanuclus pallidicinctus..... $\quad \mathbf{5 . 0 0}$

Lesser Prairie Chicken

:0s Pediœcetes pha'sianellus phasianellus ...............

Sharp-tailed Grouse

308 « Pediøectes phasianellus columbianus ................. 1.75

Columbian Sharp-tailed Grouse

3087. Pedincetes phasianellus campestris ................ 150

Prairie Sharn-tailed Grouse

309 Centrocercus urophasianus.... $\quad 3.50$ Sage Hen

:10 Meleagris gallopavo merriami.. 10.00 Merrim's 'Turkey

:10" Meleagris gallopavo silvestris.. 10.00 Wild 'Turkey

\$10h Meleagris gallopavo osceola... $\quad 5.00$ Florida 'Turkey

310r Meleagris gallopavo intermedia . 5.50 Rio Grande -Turkey

311 Ortalis vetula mccalli........ Chachalaca

312 Columba fasciata fasciata..... Band-tailed Pigeon

312" Columba .fasciata vioscr. ..... Viosca's Pigeon

$\$ 13$ Columba flavirostris.......... Red-billed Pigeon

314 Columba leucocephala.........

[314.1] Columba squamosa'........ Scaled Pigeon

315 Ectopistes migratorius........ 100.00 Passenger Pigeon

\$16 Zenaidura macroura carolinensis $\quad .10$ Mourning Dove

317 Zenaida zenaida.............

Zenaida Dove

318 Leptotila fulviventris brachyp.

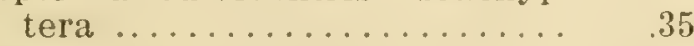

White-fronted Dove

319 Melopelia asiatica............ White-winged Dovè

320 Chæmepelia passerina terrestris .30 Ground Dove

$320 \|$ Chæmepelia passerina pallescens Mexican Ground Dove

3207 Chæmepelia passerina bermudi-

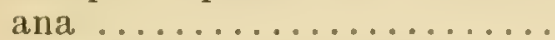
Bermuda Ground Dove 



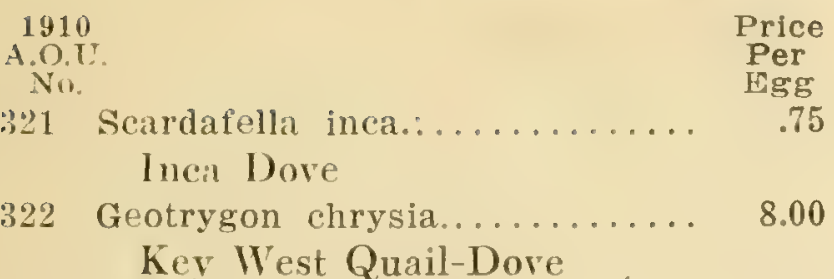

[322.1] Geotrygon montana........ 8.00

Ruddy Quail-Dore

323 Starnoena's cyanocephala....... 11.00

Blue-hended Quail-Dove

324 Gymnogyps californianus..... 750.00

California Vulture

$\begin{array}{lll}: 25 & \text { Cathartes aura septentrionalis.. } & 1.75\end{array}$

'Turkey Vulture

$:: 26$ ('atharista urubu ........... 1.50

Black Vulture

327 Elanoides forficatus......... 35.00

Swallow-tailed Kite

328 Elanus leucurus............ 25.00

White-tailed Kite

:329 Ictinia mississippiensis....... 3.50

Mississinpi Kite

330 Rostrhamus sociabilis....... 32.50

Everglade Kite

331 Circus hudsonius............ .50

Marsh Hawk

332 Accipiter velox............ 450

Sharp-shinned Hawk

333 Accipiter cooperi............ .45

Cooper's Hawk

334 Astur atricapillus atricapillus...

Goshawk

3:3 4 Astur atricapillus striatulus... 10.00

Vestern Goshawk

$: 35$ Parabuteo unicinctus harrisi... $\quad .75$

Iarris's Hawk

$::: 7$ Buteo borealis borealis........ 1.00

Red-tailed Hawk

397" Buteo borealis krideri........ 1.50

Krider's Hawk

$337 h$ Buteo borealis calurus....... $\quad .75$

Western Red-tail

$327 \pi$ Buteo borealis harlani....... $\quad 6.00$

Harlan's Hawk

$337 e$ Buteo borealis alascensis..... 6.00

Alaska Red-tail

3.39 Buteo lineatus lineatus........ $\quad .60$

Red-shouldered Hawk

359 Buteo lineatus alleni......... $\quad .95$

Florida Red-shouldered Hawk

$339 h$ Buteo lineatus elegans......... 2.00

Red-bellied Hawk

.40 Buteo abbreviatus.......... 10.00

Zone-tailed Hawk

:31 Buteo albicaudatus sennetti.... $\quad 1.40$

Sennett's White-tailed Hawk 


360 a Falco sparverius phalæna.....

Desert Sparrow Hawk

$360 b$ Falco sparverius peninsularis...

San Lucas Sparrow Hawk

$360 \mathrm{c}$ Falco sparverius paulus.......

Little Sparrow Hawk

[361] Falco sparveroides ........ 5.00

Cuban Sparrow Hawk

$36:$ Polyborus cheriway.......... 3.25

Audubon's Caracara

:6i3 Polyhorus lutosus............

Guadalupe Caracara

364 Pandion halixtus carolinensis.. $\quad 1.50$

Osprey

365 Aluco pratincola ............ .50

Barn Owl

366 Asio wilsonianus........... 45

Long-eared $\mathrm{Ow}$

$\$ 67$ Asio flammeus............ 1.00

Short-eared Owl

:68 Strix varia varia............ 1.75

Barred Owl

368 Strix varia alleni.......... 200

Florida Barred Owl

$368 b$ Strix varia albogilva......... 1.80

Texas Barred Owl

369 Strix occidentalis occidentalis... $\quad 20.00$ Spotted Owl

$369 a$ Strix occidentalis caurina..... 27.50

Northern Spotted Owl

370 Scotiaptex nebulosa nebulosa... 25.00 Great Gray .Owl

[370a] Scotiaptex nebulosa lapponica Lapp Owl

371 Cryptoglaux funerea richardsoni Richardson's Owl

372 Cryptoglaux acadica acadica... Saw-whet Owl

$372 a$ Cryptoglaux acadica scotæa... Northwestern Saw-whet Owl

373 Otus asio asio.............

Screech Owl

$\$ 784$ Otus asio fioridanus......... .55

Florida Screech Owl

3733 Otus asio mecalli.......... .50

'Texas Screech Owl

$373 c^{\circ}$ Otus asio bendirei.......... .45

California Screech Owl

$373 \prime$ Otus asio kennicotti......... 3.75

Kennicott's Screech Owl

$373 e$ Otus asio maxwelliæ......... ¿.50

Rocky Mountain Screech Owl

$373 f$ Otus asio cineraceus.......... 150

Mexican Screech Owl 

$1: 110$

A.O.U.

Price

Per

No.

$\mathrm{Egg}$

373 y Otus asio aikeni.......... 2.00

Aiken's Screech Owl

3737 Otus asio macfarlanei....... 4.00

MacFarlane's Screech Owl

373.1 Otus trichopsis............ 20.00

Spotted Screech Owl

373.2 Otus xantusi............ 20.00

Xantus's Screech Owl

374 Otus flammeolus .......... 10.00

Filimmulated Screech Owl

:374" Otus flammeolus idahoensis... 10.00

Dwarf Screech Owl

375 Bubo virginianus virginianus... $\quad 2.00$

Great Horned Owl

375 Bubo virginianus pallescens.... 1.75

Western Horned Owl
ubo virginianus subarcticus... $\quad 7.00$

$375 \%$ Bubo virginianus subarcticus...
Arctic Horned Owl

$375 e$ Bubo virginianus saturatus.... 4.00

Dusky Horned Owl

375d Bubo virginianus pacificus..... 1.75

Pacific Horned Owl

375e Bubo virginianus elachistus....

Dwarf Horned Owl

$375 f$ Bubo virginianus heterocnemis.

Labrador Horned Owl

$375 y$ Bubo virginianus algistus.....

- Saint Michael Horned Owl

376 Nyctea nyctea............ 8.00

Snowy Owl

[377] Surnia ulula ulula........ 2.50

European Hawk Owl

$377 a$ Surnia ulula caparoch.........

Hawk Owl

378 Speotyto cunicularia hypogæa.. Burrowing Owl

378ı Speotyto cunicularia floridana..

Florida Burrowing Owl

379 Glaucidium gnoma gnoma..... Pygmy Owl

379 Glaucidium gnoma californicumı. $\quad 8.00$ California Pygmy Owl

379.1 Glaucidium hoskinsi.........

Hoskins's Pygmy Owl

380 Glaucidium phaloenoides...... $\quad \mathbf{6 . 0 0}$

Ferruginous Pygmy Owl

381 Micropallas whitneyi........ 400

Elf Owl

382 Conuropsis carolinensis .......

Carolina Paroquet

382.1 Rhynchopsitta pachyrhyncha... 20.00

Thick-billed Parrot

-[383] Crotophaga ani............

Ani 

1910

A.O.U.

Price

Per

Egg

384 Crotophaga sulcirostris....... 1.25

Groove-billed Ani

385 Geococcyx californianus.......

Road-runner

386 Coccyzus minor minor.........

Mangrove Cuckoo

[386a] Coccyzus minor maynardi... $\quad 5.00$

Maynard's Cuckoo

387 Coccyzus americanus americanus

Yellow-billed Cuckoo

$387 a$ Coccyzus americanus occiden-

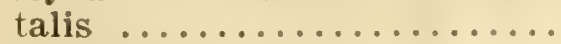

California Cuckoo

388 Coccyzus erythrophthalmus.....

Black-billed Cuckoo

[388.1] Cuculus canorus telephonus.. 10.00

Kamchatka Cuckoo

389 Trogon ambiguus............ 6.00

Coppery-tailed Trogon

390 Ceryle alcyon............. $\quad .40$

Belted Kingfisher

[390 1] Ceryle torquata.......... 8.00

Ringed Kingfisher

391 Ceryle americana septentrionalis $\quad 6.50$ Texas Kingfisher

392 Campephilus principalis...... 100.00 Ivory-billed Woodpecker

393 Dryobates villosus villosus..... $\quad .90$

Hairy Woodpecker

393॥ Dryobates villosus leucomelas.. $\quad 1.75$

Northern Hairy Woodpecker

$393 h$ Dryobates villosus auduboni...

Southern Hairy Woodpecker

393e Dryobates villosus harrisi.....

Harris's Woodpecker

393d Dryobates villosus hyloscopus.. 1.75

Cabanis's Woodpecker

\$23e Dryobates villosus monticola... 2.50

Rocky Mountain Hairy Woodpecker

$393 f$ Dryobates villosus picoideus.... 4.00

Queen Charlotte Woodpecker

393 Dryobates villosus terrænovæ... 4.00

Newfoundland Woodpecker

394 Dryobates pubescens pubescens. $\quad .75$

Southern Downy Woodpecker

394a Dryobates pubescens gairdneri.. $\quad 1.00$

Gairdner's Woodpecker

394 Dryobates pubescens homorus.. 2.00

Batchelder's Woodpecker

3940 Dryobates pubescens medianus. $\quad .75$

Downy Woodpecker

394d Dryobates pubescens nelsoni... 3.00

Nelson's Downy Woodpecker 



\begin{tabular}{|c|c|c|}
\hline $\begin{array}{l}1910 \\
\text { A.O. } \\
\text { No. }\end{array}$ & & $\begin{array}{l}\text { Price } \\
\text { Per } \\
\text { Egg }\end{array}$ \\
\hline 3940 & $\begin{array}{l}\text { Dryobates pubescens turati.... } \\
\text { Willow Woodpecker }\end{array}$ & .75 \\
\hline 395 & $\begin{array}{l}\text { Dryobates borealis............ } \\
\text { Red-cockaded Woodpecker }\end{array}$ & 3.00 \\
\hline 396 & $\begin{array}{l}\text { Dryobates scalaris bairdi...... } \\
\text { Texas Woodpecker }\end{array}$ & .50 \\
\hline $396 a$ & $\begin{array}{l}\text { Dryobates scalaris lucasanus... } \\
\text { San Lucas Woodpecker }\end{array}$ & 2.75 \\
\hline 397 & $\begin{array}{l}\text { Dryobates nuttalli............. } \\
\quad \text { Nuttall's Woodpecker }\end{array}$ & 100 \\
\hline $39 \mathrm{~S}$ & $\begin{aligned} \text { Dryobates arizonæe............. } \\
\text { Arizona }\end{aligned}$ & 6.00 \\
\hline 399 & $\begin{array}{l}\text { Xenopicus albolarvatus......... } \\
\text { White-headed Woodpecker }\end{array}$ & 2.25 \\
\hline 400 & 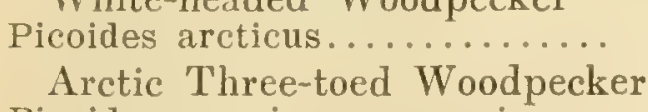 & 12.00 \\
\hline 401 & $\begin{array}{l}\text { Picoides americanus americanus } \\
\text { Three-toed Woodpecker }\end{array}$ & 12.0 \\
\hline
\end{tabular}

401" Picoides americanus fasciatus.. 18.00

Alaska Three-toed Woodpecker

4016 Picoides americanus dorsalis... 15.00

Alpine Three-toed Woodpecker

402 Sphyrapicus varius varius..... 1.00

Yellow-bellied Sapsucker

402 Sphyrapicus varius nuchalis... 1.25

Red-naped Sapsucker

403 Sphyrapicus ruber ruber...... 1.55

Red-breasted Sapsucker

403 Aphyrapicus ruber notkensis... $\quad 2.50$

Northern Red-breasted Sapsucker

404 Splyyrapicus thyroideus........ 2.00

Williamson's Sapsucker

405 Phlœetomus pileatus pileatus... 7.50

Pileated Woodpecker

405 ॥ Phløotomus pileatus abieticola. 10.00

Northern Pileated Woodpecker

406 Melanerpes erythrocephalus .... .25

Red-headed Woodpecker

407 Melanerpes formicivorus formicivorus .............. 1.00

Ant-eating IVoodpecker

$407 a$ Melanerpes formicivorus bairdi. $\quad .80$

California IVoodpecker

407b Melanerpes formicivorus angustifrons ...............

Narrow-fronted Woodpecker

408 Asyndesmus lewisi.......... $\quad .90$

Lewis's Woodpecker

409 ('enturus carolinus......... . .75

Red-bellied Woodpecker

410 Centurus aurifrons............

Golden-fronted Woodpecker

411 Centurus uropygialis..........

Gila Woodpecker
3.50 

412 Colaptes auratus auratus...... . .10

Flicker

412 "Colaptes auratus luteus....... .10

Northern Flicker

413 Colaptes cafer collaris.......... .15

Red-shafted Flicker

$41:$. Colaptes caler saturatior...... . .

Northwestern Flicker

414 Colaptes chrysoides............ . .i. Gilded Flicker

415 ('olaptes rufipileus............ 16.50)

Guadalup? Ilicker

416 Antrostomus carolinensis ..... 2.75

Chuck-will's Widow

417 Antrostomus vociferus vocilerus

Whip-poor-will

$417 "$ Antrostomus vociferus macromystax ............. 12.00

Stephens's Whip-poor-will

418 Phalænoptilus nuttalli nuttalli. 1000 Poor-wil]

418ı Phalænoptilus nuttalli nitidus. Frosted Poor-will

4181) Phalænoptilus nuttalli californicus ............... 12.50

Dusky Poor-will

419 Nyctidromus albicollis merrilli. 4.00 Merrill's Parauque

420 Chordeiles virginianus virginianus .................60

Nighthawk

$420 a$ Chordeiles virginianus henryi...

Western Nighthawk

4201 Chordeiles virginianus chapmani Florida Nighthawk

$420 c$ Chordeiles virginianus sennetti. Sennett's Nighthawk

420 Chordeiles virginianus hesperis Pacific Nighthawk

421 Chordeiles acutipennis texensis. Texas Nighthawk

422. Cyseloides niger borealis...... 75.00 Black Swift

423 Chætura pelagica............ $\quad .40$

Chimney Swift

424 Chætura vauxi.

Vaux's Swift

425 Aëronautes melanoleucus....... 10.00

White-throated Swift

426 Eugenes fulgens............ 7.50

Rivoli's Hummingbird

427 Cyanolæmus clemenciæ....... 50.00 Blue-throated Hummingbird 

428 Archilochus colubris........... 1.25

Ruby-throated Hummingbird

429 Archilochus -alexandri.........

Black-chinned Hummingbird

430 Calypte costre..............

Costa's Hummingbird

4:1 calypte anna..............

Anna's Hummingbird

4::2 Selasphorus platycercus.

Broad-tailed Hummingbird

493: Selasphorus rufus.............

Rufous Hummingbird

434 Selasphorus alleni............

Allen's Hummingbird

4:5 Atthis morcomi.............

Morcom's Hummingbird

$4: 36$ Stellula calliope.........

Calliope Hummingbird

4::7 ('alothorax lucifer.

Lucifer Hummingbird

4 4:S Amizilis tracatl.............

Rieffer's Hummingbird

439 Amizilis cerviniventris chalcon-

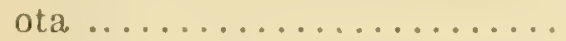

Buff-bellied Hummingbird

439.1 Uranomitra salvini... . . . . . . .

Salvin's Hummingbird

440 Basilinna xantusi............

Xantus's Hummingbird

440.1 Basilinna leucotis.

White-eared Hummingbird

441 Cynanthus latirostris......... 10.00

Broad-billed Hummingbird

[441.1] Platypsaris a'glaiæe albiventris 10.00

Xantus's Becard

[442] Muscivora tyrannus......... 3.00

Fork-tailed Flycatcher

443 Muscivora forficata........... .25

Scissor-tailed Flycatcher

444 Tyrannus tyrannus............ $\quad .10$

Kingbird

445 Tyrannus dominicensis....... 2.25

Gray Kingloird

446 Tyrannus melancholicus couchi. 1.25

Couch's Kingbird

447 Tyrannus verticalis........... 15

Arkansas Kingbird

448 Tyrannus vociferans.......... .25

Cassin's Kingbird

449 Pitangus sulphuratus derbianus 2.25

Derby Flycatcher

451 Myiodynastes luteiventris...... 6.00

Sulphur-bellied Flycatcher 

1910

A. O.U.

Price

Per

10.

.35

45: Myinroins reinitus..........

Crested Flycatcher

453 Myiarchus magister magister...

2.50

Arizona Crested Flycatcher

453 Myiarchus magister nelsoni...

Mexican Crested Flycatcher

454 Myiarchus cinerascens cineras cens:

Ash-throated Flycatcher

4541. Myiarchus cinerascens pertinax

Lower California lilycatcher

155 Myiarchus lawrencei olivascens

Olivaceous Flycatcher

biti sidvernic phomb $\ldots \ldots \ldots \ldots \ldots$

Plinebe

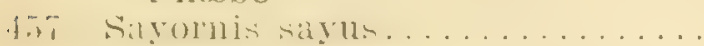

Say's Plonebe

4.5 sigulnit nigricant

Black Phoebe

459 Nuttallornis borealis.........

Olive-sided Flycatcher

460 Myiochanes pertina'x pallidiventris ................... 300

Coucs's I'lycatcher

461 Myioclianes virens...........

lVood Pewee

46. Vyiochanes richardsoni richard-

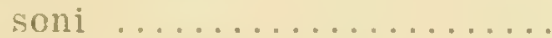

Western Wood Pewee

16"2" Myiochanes richardsonj peninsule . . . . . . . . . . . .

I allow-billed Irood Pewee

16:- limpidonax flaviventris........

Vellow-bellicd Flycatcher

10.00

fir mmpidonax difficilis difficilis... Vestern Flycatcher

lit" wmpidonax difficilis cineritius. . Sian Lacis Flycatcher

465 Gmpidonax virescens......... Acildinn FIycatcher

5.00

466 Empidonax trailli trailli.......

'Traill's Flycatcher

466 . Empidonax trailli alnorum.....

Alder Flycatcher

467 Empidonax minimus..........

Least Flycatcher

468 Empidonax hammondi.........

Hammond's Flycatcher

469 Empidonax wrighti...........

Wright's Flycatcher

4631 Empidonax griseus

Gray Flycatcher

470 Empidonax fulvifrons pygmæus 10.00 Buff-breasted Flycatcher 

1910

A.O.U.

No.

Price

Per

Egg

471 Pyrocephalus rubinus mexicanus

1.00

Vermilion Flycatcher

472 Camptostoma imberbe........

Beardless Flycatcher

[473] Alauda arvensis............

Skylark

474 Otocoris alpestris alpestris....

Horned Lark

$474 \iota$ Otocoris alpestris arcticola....

Pallid Horned Lark

474h, Otocoris alpestris praticola.... Prairie Horned Lark

$474 c$ Otocoris alpestris leucolæma...

Desert Horned Lark

474, Otocoris alpestris giraudi.....

'Texas Horned Lark

$474 e$. Otocoris alpestris actia........

California Horned Lark

$474 f$ Otocoris alpestris rubea.......

Ruddy Horned Lark

474! Otocoris alpestris strigata.....

Streaked Horned Lark

4747 . Otocoris alpestris adusta...... 1.50

Scorched Horned Lark

$474 i$ Otocoris alpestris merrilli..... 1.50

Dusky Horned Lark

474j Otocoris alpestris pallida...... 2.50

Sonora Horned Lark

474k: Otocoris alpestris hoyti...... 2.75

Hoyt's Horned Lark

474l. Otocoris alpestris occidentalis .. 2.00

Montezuma Horned Lark

$474 \mathrm{~m}$. Otocoris alpestris insularis....

3.00

Island Horned Lark

475 Pica pica hudsonia...........

Magpie
476 Pica nuttall

Yellow-billed Magpie

1.00

477 Cyanocitta cristata cristata....

Blue Jay

477 Cyanocitta cristata florincola...

Florida Blue Jay

478 Cyanocitta stelleri stelleri.....

Steller's Jay

478 " Cyanocitta stelleri frontalis....

Blue-fronted Jay

478/ Cyanocitta stelleri diademata...

Long-crested Jay

478e Cyanocitta. stelleri annectens...

Black-headed Jay

478 Cyanocitta stelleri carlottæ.....

Queen Charlotte Jay

478e Cyanocitta stelleri carbonacea. . 

1910

A.O.U.

No

Price

479

phelocoma cyanea.........

Per

Egg

Florida Jay

480 Aphelocoma woodhousei.......

IVoodhou'se's Jay

480.1 Aphelocoma cyanotis.........

Blue-eared Jay

6.00

480.2 Aphelocoma texana..........

6.00

'Texas Jay

481. Aplielocoma californica californira. . . . . . . . . . . . . . .

California diy

SSlu Aphelocomat calitornica liypo-

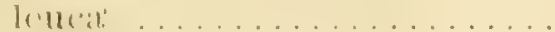

Xantus's . Iay

1816 Aphelocoma califormia obscura.

Bclding's J Jy

$4 \$ 1.1$ Aplelocoma insularis.........

Siantar Cru\% Jay

45\% Aplolocoma sieberi arizona...

Arizonia Jay

482" Aphelocoma sieberi couchi....

Couch's Jay

483 Xanthoura luxuosa glaucescens.

Green Jay

484 Perisoreus canadensis canadensis .................. 20.00

Canada Jay

6.00

1.50

2.30

1.00

6.00

1.50

484" Perisoreus canadensis capitalis. 18.00

Rocky Mountain Jay

484h Perisoreus canadensis fumitrons

Alaska Jay

4840 l’erisoreus canadensis nigricapil-

lus

Labrador Jay

485 Perisoreus obscurus obscurus.. 25.00

Oregon Jay

485n Perisoreus obscurus griseus.... 27.50

Gray Jay

486 Corvus corax sinuatus....... 3.75

Raven

4S6" Corvus corax principalis......

6.00

Northern Raven

487 Corvus cryptoleucus.......... .75

IVhite-necked Raven

488 Corvus brachyrhynchos brachy-

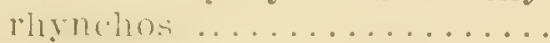

Crow

488 " Corvus brachyrhynchos pascuus

Florida Crow

4881) Corvus brachyrhynchos hesperis Western Crow

489 Corvus caurinus............

Northwestern Crow

490 Corvus ossifragus............

Fish Crow 



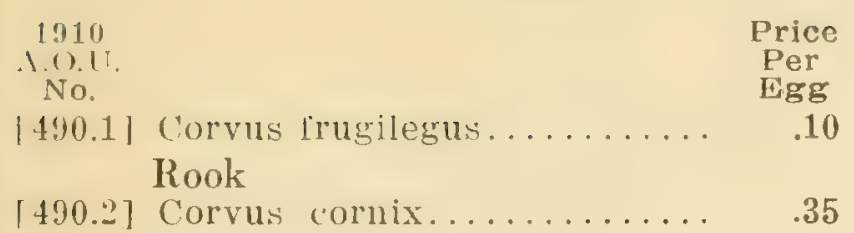

Hooded Crow

491 Nucifraga columbiana........ 25.00

Clarke's Nutcracker

492 Cyanocephalus cyanocephalus.. $\mathbf{2 . 2 5}$

Pinon Jay

498 Sturnus vulgaris..........

Starling

494 Dolichonyx oryzivorus....... .50

Bobolink

495 Molothrus ater ater......... . .10

Cowbird

495a Molothrus ater olscurus...... $\quad .20$

Dwarf Cowbird

496a Tangavius æneus æneus...... .75

Bronzed Cowbird

496 Tangavius aneus involucratus.. $\quad .50$

Red-eyed Cowbird

497 Xanthocephalus xanthocephalus. $\quad .10$

Yellow-headed Blackbird

498 Agelaius phœniceus phœniceus. $\quad .10$

Red-winged Blackbird

498a Agelaius phœniceus sonoriensis. $\quad .15$

Sonora Red-wing

4986 Agelaius phœeniceus bryanti... $\quad 2.00$

Bahama Red-wing

498c Agelaius phøeniceus floridanus.. .30

Florida Red-wing

498 Agelaius phøiceus fortis..... $\quad .35$

Thick-billed Red-wing

498e Agelaius phœniceus neutralis... $\quad .10$

San Diego Red-wing

$498 f$ Agelaius phœniceus caurinus...

Northwestern Red-wing

498") Agelaius phœniceus richmondi..

Vera Cruz Red-wing

499 Agelaius gubernator californicus .15

Bicolored Red-wing

500 Agelaius tricolor ............ $\quad .15$

Tricolored Red-wing

501 Sturnella magna magna...... .25

Meadowlark

501» Sturnella magna hoopesi...... $\quad .50$

Rio Grande Meadowlark

501c Sturnella magna argutula..... .50

Southern Meadowlark

501.1 Sturnella neglecta...........

Western Meadowlark

503 Icterus melanocephalus auduboni

Audubon's Oriole

504 Icterus parisorum...........

Scott's Oriole 

1910

A.O.U.

Price

Per

505 Icterus cuculiatus sennetti.....

$\mathrm{E}$ ing

.65

Sennett's Oriole

505 Icterus cucullatus nelsoni.....

Arizona Hooded Oriole

506 Icterus spurius.............

Orcliard Oriole

507 Icterus galbula ............

Baltimore Oriole

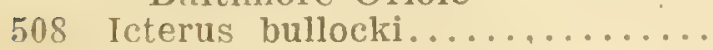

Bullock's Oriole

509 Eupliagus carolinus..........

Rusty Blackbird

510 Euphagus cyanocephalus.......

Brewer's Blackbird

511 Quiscalus quiscula...........

Purple Grackle

511" Quiscalus quiscula aglæus.....

Florida Grackle

5117, Quiscalus quiscula æneus......

Bronzed Grackle

513 Megaquiscalus major major....

Boat-tailed Grackle

513 k Megaquiscalus major macrourus

Great-tailed Grackle

514 Hesperiphona vespertina vespestina .................

Evening Grosbeak

514" Hesperiphona vespertina mon-

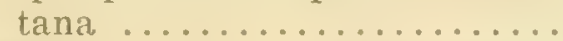

Western Evening Grosbeak

515 Pinicola enucleator leucura..... 15.00

Pine Grosbeak $\quad * 5,00$

515 \& Pinicola enucleator montana... 20.00

Rocky Mountain Pine Grosbeak

515h Pinicola enucleator californica.. . 20.00

California Pine Grosbeak

515c Pinicola enucleator alascensis..

Alaska Pinc Grosbeak

515r Pinicola enuclcator flammula...

Kiadiak Pine Grosbeak

|5lif Pyrrhula cassini.............

Cassin's Bullfinch

517 Carpodacus purpureus purpureus Purple Finch

517 a Carpodacus purpureus californi(:11s ..................

California Purple Finch

518 Carpodacus cassini...........

Cassin's Purple Finch

519 Carpodacus mexicanus frontalis Honse Finch

5196 Carpodacus mexicanus ruberri-

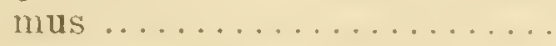

San Lucas House Finch 

1910

A.O.U.

No.

Price

Per

Egg

519c Carpodacus mexicanus clementis

1.00

San Clemente House Finch

520 Carpodacus amplus...........

Guadalupe House Finch

520.1 Carpodacus megregori........

McGregor's House Finch

6.00

5.75

521 Loxia curvirostra minor....... Crossbill

521 ( Loxia curvirostra stricklandi... Mexican Crossbill

52: Loxia leucoptera.............

White-winged Crossbill

523 Leucosticte griseonucha....... Aleutian Rosy Finch

524 Leucosticte tephrocotis tephro-

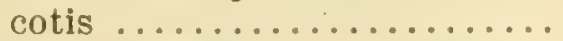

Gray-crowned Rosy Finch

$524 a$ Leucosticte tephrocotis littoralis Hepburn's Rosy Finch

525 Leucosticte atrata............ Black Rosy Finch

526 Leucosticte australis..........

Brown-capped Rosy Finch

527 Acanthis hornemanni horneman-

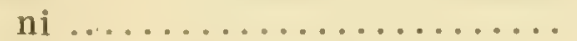

Greenland Redpoll

$527 "$ Acanthis hornemanni exilipes.. Hoary Redpoll

15.00

25.00

18.00

20.00

4.00

5.00

2.00

.35

528 " Acanthis linaria holbœlli..... $\quad 5.00$

Holböll's Rednoll

$528 \%$. Acanthis linaria rostrata...... $\quad 6.00$

Greater Redpoll

529 Astragalinus tristis tristis..... $\quad .20$

Goldfinch

529a Astragalinus tristis pallidus... Pale Goldfinch

$529 h$ Astragalinus tristis salicamans..

IVillow Goldfinch

530 Astragalinus psaltria psaltria... Arkansas Goldfinch

530a Astragalinus psaltria hesperophilus ............... $\quad .20$

Green-backed Goldfinch

531 Astragalinus lawrencei....... $\quad .50$

Lawrence's Goldfinch

[532] Spinus notatus............ 250

Black-headed Goldfinch

533 Spinus pinus............. 1.80

Pine Siskin

534 Plectrophenax nivalis nivalis... $\quad \mathbf{3 . 0 0}$

Snow Bunting $\quad 1.50$ 

A.O.U.
No.
$534 \because$ Plectrophenax nivalis townsendi
$\quad$ Pribilof Snow Bunting

535) Plectrophenax hyperboreus....

Mchay's Snow Bunting

$5: 36$ Calcarius lapponicus lapponicus.

Lapland Longspur

5:36r Calcarius lapponicus alascensis.

Miskia Longspur

$5: 7$ Calcarius pictus............

Simith's Longspux

5::s calcarius ornatus............

Chestnut-collared L.ongspur

5::) Rlyynchophanes mccowni......

Mc C'own's L,ong'spur

540 l'ooecotes gramineus graminus.

Vesper sparrow

5401 l'onecetes gramineus confinis...

Wrestern Vesper. Siparrow

5406 l'oncotes gramineus affinis....

Oregon Vesper Sparrow

541 l'asserculus princeps.........

Ipswich Sparrow

542 Passerculus sandwichensis sandwichensis

Aleutian Sarannah Sparrow

$542 \|$ Passerculus sandwichensis savamna ................

Savannal Sparrow

5427 Passerculus sandwichensis alaudinu. ...................

Western Sarannah Sparrow

5420 Passerculus sandwichensis bry anti ${ }^{\circ}$ and

\section{Bryant's Sparrow}

54\% Passérculus beldingi............ Belding's Sparrow

544 Passerculus rostratus rostratus. Large-billed Sparrow

544ı. Pa'sserculus rostratus guttatus. San Lucas Sparrow

544r Passerculus rostratus sanctorum

San Benito Sparrow

545 Ammodramus bairdi..........

$$
\text { Baird's Sparrow }
$$

546 Ammodramus savannarum australis ...................

Grasshopper Sparrow

546" Ammodramus savannarum bi-

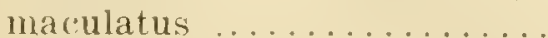

Vestern Grasshopper Sparrow

5467 Ammodramus savannarum floridanus ...........................

Florida Grasshopper Sparrow
Price

Per

$\mathrm{Eg} g$

5.00

30.00

3.00

1.50

$\$ .80$

6.00

$\therefore i 5$ 

1910

A.O.U.

No.

547 Passerherbulus henslowi lien=

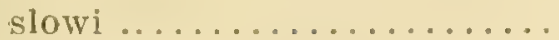

Henslow's Sparrow

547 " Passerherbulus henslowi occidentalis .................

Western Henslow's Sparrow

"4s Passerherbulus lecontei....... L.econte's Sparrow

54!) Passerherbulus caudacutus..... Sharp-tailed Sparrow

549.1 Passerherbulus nelsoni nelsoni. Nelson's Sparrow

549.1" Passerherbulus nelsoni subvir-

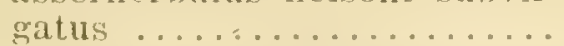

Acadian Sharp-tailed Sparrow

550 Passerlerluulus maritimus maritimus .................

Seaside Sparrow

5504 Passerherbulus maritimus penin-

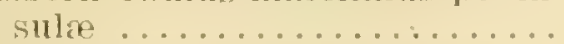

Ścott's Seaside Sparrow

5nof Passerherbuhs maritimus sen-

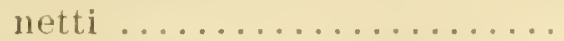

'l'exas Seaside Sparrow

550 r Passerherbulus maritimus fisheri Louisiana Seaside Sparrow

Price

Per

Egg

2.50

6.00

4.50

20.00

iil)

4.00

4.00

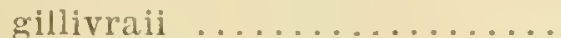

Macgillivray's Seaside Sparrow

550 r Passerherbulus maritimus mac.

551 Passerherbulus nigrescens..... 20.00

Dusky Seaside Sparrow

552 Chondestes grammacus grammacuc.

Lark Sparrow

5.52 11 Chondestes grammacus strigatus

Western Lark Sparrow

5.;:) \%onotrichia ruerula..........

Harris's Sparrow

554 Zonotrichia leucophrys leucoph-

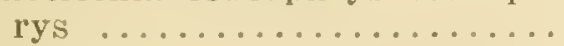

White-crowned Sparrow

Gambel's Sparrow

5547 Zonotrichia leucophrys nuttalli. Nuttall's Sparrow

5.57 7.0motrichia coronata

Golden-crowned Sparrow

558 Zonotrichia albicollis .....

White-throated Sparrow

4.00

'I'ree Sparrow

559 " . Spizella monticola ochracea...

Western Tree Sparrow

5.00

560 Spizella passerina passerina...

Chipping Sparrow 

1910

A.O.U.

Price

Per

No.

Egg

$560 n$ Spizella passerina arizonæ.....

. 10

Western Chipping Sparrow

..61 spizella pallida............. .60

Clay-colored Sparrow

562 Spizella breweri............

Brewer's Sparrow

563 Spizelia pusilla pusilla.......

Field Sparrow

563 Spizella pusilla arenacea......

ITestern Field Sparrow

5,4 Spizella wortheni...........

Worthen's Sparrow

565 Spizella atrogularis...........

Black-chinned Sparrow

566 Junno aikeni...............

White-winged Junco

567 Junco hyemalis hyemalis......

Slate-colored Junco

567 ॥unco hyemalis oreganus......

Oregon Junco

567h) Junco hyemalis connectens.....

Shufeldt's Junco

567 e Junco hyemalis thurberi.......

'Thurber's Junco

5671 Junco hyemalis pinosus. . . . . .

Point Pinos Junco

567e Junco liyemalis carolinensis....

Carolina Junco

567 J Junco hyemalis montanus......

Montana Junco

567 " Junco hyemalis mearnsi.......

Pink-sided Junco

5677 Junco hyemalis annectens.....

Ridgway's Junco

$567 i$ Junco hyemalis townsendi.....

'Townsend's Junco

570 Junco phæonotus palliatus....

Arizona Junco

570 " Junco phæonotus dorsalis.....

Red-backed Junco

1.00

.10

1.50

3.50

2.00

1.00

1.50

1.00

5706. Junco phæonotus caniceps.....

Gray-headed. Junco

571 .Junco bairdi...............

Baird's Junco

572 Junco insularis.

Guadalupe-Junco

573 Amphispiza bilineata bilineata. .

Black-throated Sparrow 

574.1 Amphispiza nevadensis nevaden-

Per

Egg sis .................. 200

Sage Sparrow

574.1" Amphispiza nevadensis cinerea' Gray Sage Sparrow

574.17 Amphispiza nevadensis canes('ells ............... 2.25

California Sage Sparrow

575 Pucrea astivalis restivalis.... Pine-woods Sparrow

575 " Pencra æestivalis bachmani.... Bachman's Sparrow

576 l'cucrea botterii.

Botteri's Sparrow

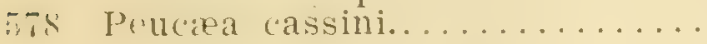

Cassin's Sparrow

579 Aimophila carpalis.

Rufous-winged Sparrow

5 so Aimophila ruficeps ruficeps..... Rufous-crowned Sparrow

580 " Aimophila ruficeps scotti...... Scott's Sparrow

5807, Aimophila ruficeps eremœea... Rock Sparrow

580 r Aimophila ruficeps sororia..... Laguna Sparrow

581 Melospiza melodia melodia.... Song Sparrow

581، Melospiza melodia fallax......

Desert Song Sparrow

581h Melospiza melodia montana....

Mountain Song Sparrow

5sir Melospiza melodia heermanni...

Heermann's Song Sparrow

581r Melospiza melodia' samuelis....

Samuels's Song Sparrow

581, Melospiza melodia morplina....

Rusty Song Sparlow

$581 f$ Melospiza melodia rufina..... Sooty Song Sparrow

581!/ Melospiza melodia rivularis.... Brown's Song Sparrow

5817 . Melospiza melodia graminea... Santa Barbara Song Sparrow

581i Melospiza melodia clementæ... San Clemente Song Sparrow

581 i Melospiza melodia juddi....... Dakota Song Sparrow

581/: Melospiza melodia' merrilli..... Merrill's Song Sparrow

5817. Melospiza melodia pusillula.... Alameda Song Sparrow 

1910

1. O.U.

No.

Price

Pel

581" Melospiza melodia caurina....

Egg

Yakutat Song Sparrow

581n Melospiza melodia kenaiensis...

Kenai Song Sparrow

5S1》 Melospiza melodia cleonensis...

Mendocino Song Sparrow

581" Melospiza melodia insignis....

4.00

Bischoff"s Song Sparrow

$581 \%$ Melospiza melodia sanaka.....

Aleutian Song Sparrow

5\$1 Melospiza melodia maxillaris...

Suisun Song Sparrow

583 Melospiza lincolni lincolni.....

Lincoln's Sparrow

583 " Melospiza lincolni striata......

6.00

forbush's Sparrow

$5 \$ 4$ Melospiza georgiana..........

Swamp Sparrow

585 Passerella iliaca iliaca........

Fox Sparrow

$585 \|$ Passerella iliaca unalaschcensis

6.00

Slimmgin liox Spurrow

585/, Passerella iliaca megarhnycha..

3.50

Thick-billed fox Sparrow

5850 Passerella iliaca schistacea....

Slate-colored Fox Sparrow

585d Passerella iliaca stephensi.....

Stephens' lox Sparrow

6.50

5850 Passerella iliaca fuliginosa.....

Sooty liox Sparrow

$585 f$ Passerella iliaca insularis......

Kadiak Fox Sparrow

$585 g$ Passerella iliaca townsendi....

'Townsend's Fox Sparrow

586 Arremonops rufivirgatus.......

Texas Sparrow

587 Pipilo erythrophthaImus ery-

throphthatmus ............. .

'lowhee

587ィ Pipilo erythrophthalmus alleni..

IT'hite-eyed 'Towhee

588 Pipilo maculatus arcticus..... 125

Aretic 'Towhee

588 " Pipilo maculatus montanus....

Spurred 'Towhee

58s/ Pipilo macula'tus oregonus......

Oregon Towhee

5ss: Pipilo maculatus clementæ....

San Clemente 'I'owhee

588d. Pipilo maculatus megalonyx...

San. Diego Towhee

588ë Pipilo maculatus magnirostris.

Large-billed 'Towhee 

1910

A.O.U.

Price

Per

No.

EgE

$5 s 9$ Pipilo romsobrinus...........

Guadalupe Towhee

591 Pipilo fuscus mesoleucus.......

Canon Towhee

591" Pipilo fuscus albigula.........

San Lucas Towhee

591.1 Pipilo crissalis crissalis......

California Towhee

591.1" Pipilo crissalis senicula......

Anthony's Towhee

592 I'ipilo aberti..............

Abert's 'Towhee

592.1 Oreospiza chlorura...........

Green-tailed 'Towhee

593 Cardinalis cardinalis cardinalis. Cardinal

593 đardinalis cardinalis superbus. . Arizona Cardinal

5936 Cardinalis cardinalis igneus.... . San Lucas Cardinal

593 c Cardinalis cardinalis canicaudus $\quad .40$

Gray-tailed Cardinal

593 Cardinalis cardinalis floridanus. $\quad .75$

Florida Cardinal

594 Pyrrhuloxia sinuata sinuata... 2.50

Arizona Pyrrhuloxia

594 Pyrrhuloxia sinuata texana....

'Texas Pyrrhuloxia

$594 b$ Pyrrhuloxia sinuata peninsulæe.. $\quad 5.00$

San Lucas Pyrrhuloxia

595 Zamelodia ludoviciana........ .25

Rose-breasted Grosbeak

596 Zamelodia melanocephala....... .20

Black-headed Grosbeak

597 Guiraca cærulea cærulea...... $\quad .60$

Blue Grosbeak

$597 \_$Guiraca cæerulea lazula....... $\quad .70$

Western Blue Grosbeak

598 Passerina ryanea............ .20

Indigo Bunting

599 Passerina amœna........... $\quad .20$

Lazuli Bunting

600 Passerina versicolor versicolor.. $\quad 4.00$

Varied Bunting

$600 \pi$ Passerina versicolor pulchra... $\quad 6.00$

Beautiful Bunting

601 Passerina ciris............. . .20

Painted Bunting

602 Sporophila morelleti sharpei... $\quad 3.50$

Sharpe's Seedeater

[60:3] Tiaris hicolor.............. $\quad 3.00$

Grassquit

[60:3.1] Tiaris canora........... 10.00

Melodious Grassquit 

$1: 110$

A.O. U.

No.

(i) 1

Sillizit almerixanil.

Dickcissel

605 Calamospiza melanocorys...... Lirk Bunting

607 riranga ludoriciand.

IVestern 'Tanager'

608 Piranga erythromelas.........

Scarlet Tanager

609 Piranga lepatica............

Hepatic Tanager

610 Pimanga rubra rubra.

Summer 'Tanager'

610 ( Piranga rubra cooperi.........

Cooper's Tanager

611 Progne subis subis..........

Purple Martin

611 ( Progne subis hesperia.........

ITestern Martin

611.1 Progne cryptoleuca...........

Cuban Martin

611.: Progne chalybra............

Gray-breasted Martin

612 Petrochelidon lunifrons lunifrons

Cliff Swallow

612" Petrochelidon lunitrons tachina.

Lesser Cliff Swallow

612l, Petrochelidon lunifrons melano-

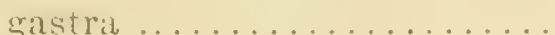

Mexican Cliff Srrallow

[612.1] Petrochelidon fulva.........

Cuban Cliff Swallow

613 Hirundo erythrogastra........ Barn Swallow

[61:.1] Hirundo rustica............ Guropean Swallow

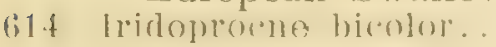
'Tree Sivallow

615 Tachycineta thalassina lepida...

Northeru Violet-rreen Swallow

615" 'achycineta thalassina brachyptera............... 50

San Lucas Swallow

[615.1] Callichelidon cyaneoviridis... 6.00 Ballanil Swallow

[615.2] Chelidonavia urbica........ .20 European Martin

616 Riparia riparia............... 10 Bank Sivillow

617 Stelgidopteryx serripennis..... Rough-winged Swallow

61s Bombycilla garrula......... 25.00 Bollemian Waxwing *8.00

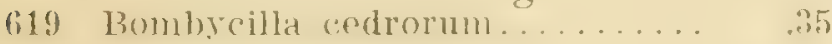

Per

Egg 

620 Phainopepla nitens..........

Per

Egg

Phainopepla

621 Lanius borealis..............

Northern Shrike

622 Lanius ludovicianus ludovicianus

Loggerhead Shrike

622 Lanius ludovicianus excubito-

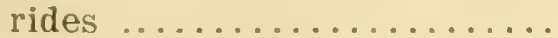

White-rumped Shrike

622" Lanius ludovicianus gambeli...

California Shrike

622 Lanius ludovicianus anthonyi...

Island Shrike

$622 e$ Lanius ludovicianus migrans....

Migrant Shrike

623 Vireosylva calidris barbatula...

Black-whiskered Vireo

624 Vireosylva olivacea...........

Red-eyed Vireo

[625] Vireosylva flavoviridis........

Yellow-green Vireo

626 Vireosylva philadelphica ......

Philadelphia Vireo

627 Vireosylva gilva gilva....... $\quad .50$

Warbling Vireo

627 " Vireosylva gilva swainsoni....

Western Warbling Vireo

628 Lanivireo flavifrons........... $\quad .75$

Yellow-throated Vireo

629 Lanivireo solitarius solitarius...

Blue-headed Vireo

629" Lanivireo solitarius cassini.....

1.00

Cassin's Vireo

629 banivireo solitarius plumbeus..

Plumbeous Vireo

6290 Lanivireo solitarius alticola....

Mountain Vireo

629r Lanivireo solitarius Iucasanus..

San Lucas Vireo

630 Viren atricapillus.

Black-capped Vireo

631 Vireo griseus griseus.........

White-eyed Vireo

631 Vireo griseus maynardi.......

Key IVest Vireo

631h Vireo griseus bermudianus.....

Bermuda Vireo

631" Vireo griseus micrus..........

Small White-eyed Vireo

632 Vireo huttoni huttoni..........

Hutton's Vireo

632 aireo huttoni stephensi........ 

632. Vireo huttoni obscurus........

$$
\text { Anthony's Vireo }
$$

6:32. Vireo liutioni cognatus........

$$
\text { Frizal's Vireo }
$$

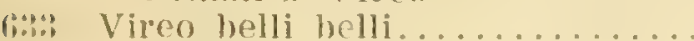

$$
\text { Bell's Vireo }
$$

(i:2:)!n Vireo belli pusillus.

l.cist Vireo

(i:3)h Viren helli medius ..........

lexis Vireo

6i:4 Vireo vicinior

(imar Vireo

6:15 coreba bahamensis..........

Ballama Honey Creeper

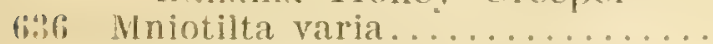

Blick and White Mrabler

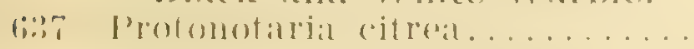

Prothomotary Wirbler

lios Helinala swainsoni...........

Siwainson's Mrabler

(i:s!) Holmitheros vermivorus........

Worm-cating Mrabler

fi40 Vermivora bachmani.........

Bachman's Warbler

b41 Fimivora pinus.

Blue-winged. Warbler

642 Vermivora chrysoptera........

Golden-winged IV arbler

64:. Trunivora lucia ............

Lucy's Warbler.

644 Vermivora virginiæ...........

Virginia's IVarbler

645 Vermivora rubricapilla rubrica-

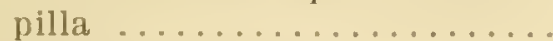

Nashrille Marbler

645 ( Vermivora rubricapilla guttura-

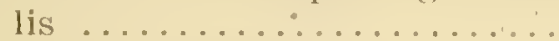

Calaveras IVarbler

646 Vermivora celata celata....... Orange-crowned Warbler

646، Vermivora celata lutescens.... Lutescent Warbler

6461 Vermivora celata sordida...... Dusky IVarbler

647 Vermivora peregrina

'Tennessee, Warbler.

648 Compsothlypis americana ameri-

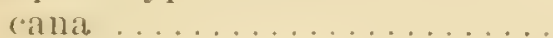
Parula IVarbler

648، Compsothlysis americana usnere Northern Parula Warbler 649 Compsothlypis pitiayumi nigri-

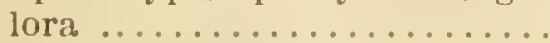

Sennett's Warbler 

1910

A. O. U.

No.

650

Dendroica tigrina.

Cipe Mar Warbler

651 Peucedramus olivaceus.........

Olive Mrabler

6.52 Dendroica restiva astiva....... rellow Mrabler

652" Dendroica astiva sonorana.... Sonoria Yellow Wrabler

6526 Dendroica restiva rubiginosa...

Alusk Y Yellow Warbler

652r Dendroica astiva brewsteri....

California Yellow Wabler

653 Dendroica bryanti castaneiceps. Mangrove IVabler

654 Dendroica carulescens carules-

Blinck-tlinoated Blue Warbler

65.4" Dendroica caerulescens cairusi.

Cams"s $\|^{T}$ arbler"

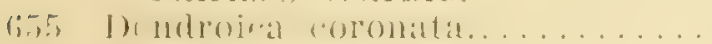
Mrrtle Warbler

656 Dendroica auduboni auduboni... Audubon's IVirbler

656" Dendroica auduboni nigrifrons.. Black-fronted Wrabler

657 Dendroica magnolia......... Magnolia IVarbler

658 Dendroica cerulea............ Cerulean Warbler

659 Dendroica pensylvanica........ Chestnut-sided IVarbler

660 Dendroica castanea........... Bay-breasted Warbler

661 Dendroica striata............ Black-poll IVarbler

662 Dendroica fusca............

Blackburnian IVarbler

663 Dendroica dominica dominica...

Yellow-throated IVarbler

663 n Dendroica dominica albilora.... Sycamore Warbler

664 Dendroica gracia Grace's IVarbler

665 Dendroica nigrescens.......... Black-throated Gray Warbler 666 Dendroica chrysoparia......... Golden-cheeked Marbler

667 Dendroica virens............

Black-throated Green Warbler 668 Dendroica townsendi........ 27.50

'Townsend's Warbler

669
Price

Per

Egg

25.00

25.00

.10

.50

2.50

.15

20.00

$\because, 00$

3.00

8.00

3.00

6.00

20.00

2.50

6.00

25.00
Dendroica occidentalis.........

Hermit IVarbler 

1910

A.O.U.

Price

Per

No.

Egg

670 Dendroica kirtlandi

27.50

Kirtland's Warbler

671 Dendroica vigorsi............

Pine $\mathbf{T}$ arbler

672 Dendroica palnarum palmarum. 10,00

Palm Warbler

672 dendroica palmarum hypochry-

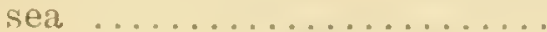

Y'tlow Palm IVarbler

6.00

673 Dendroica discolor............

Prairie Warbler

674 Seiurus aurocapillus.......... Oren-bird

675 Seiurus noveboracensis novebor-

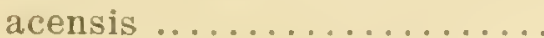

Water-Thrush

$675 a$ Seiurus noveboracensis notabilis .............. 6.00

Grinnell's Water-'Thrush

(:i) Seiurus motacilla.

Louisiana Water-Thrush

677 Oporornis formosus...........

Kentucky Warbler

1.00

678 Oporornis agilis

30.00

Connecticut Warbler

679 Oporornis philadelphia........

5.00

Mourning IVarbler

$680^{\circ}$ Oporornis tolmiei.

Macgillivray's Warbler

681 Geothlypis trichas trichas.....

Maryland Yellow-throat

$6 \$ 1 "$ Geothlypis trichas occidentalis..

Western Yellow-throat

6817 Geothlypis trichas ignota......

Florida Yellow-throat

681e Geothlypis trichas arizela'...... Pacific Yellow-throat

$681 e$ Geothlypis trichas sinuosa......

Salt Marsh Yellow-throat

682 Geothlypis beldingi...........

Belding's Yellow-throat

682.1 Chamæthlypis poliocephala.....

5.00

Rio Grande Yellow-throat

$6 \$ 3$ Icteria virens virens..........

Yellow-breasted Chat

$683 a$ Icteria virens longicauda.......

Long-tailed Chat

64 Wilsonia citrina.............

Hooded Warbler

685 Wilsonia pusilla pusilla....... 10.00

Wilson's Warbler

685 Wilsonia pusilla pileolata......

Pileolated Warbler 



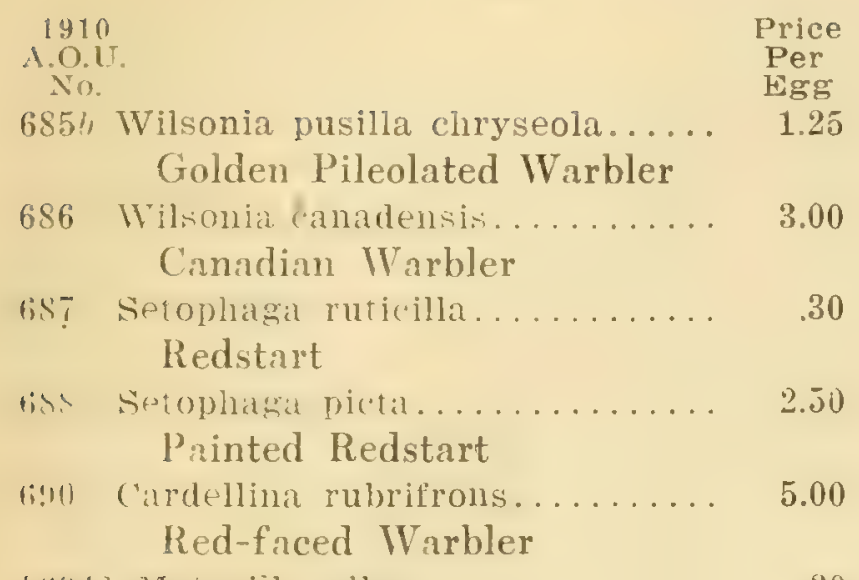

[ti!t] Motacilia alba............ .20

White Wagtail

[b95] Motacilla oculuris...........

Swinhoe's Wagtail

696 Budytes Havus alascensis...... 10.00

Alaska Yellow Wagtail

697 Anthus rubertens............

Pipit

[ $6: 9$, ] Anthus prattensis...........

Meadow Pipit

[6:9] Anthus cervinus.

Red-throated Pipit

io0 Anthus spraguei.............

Sprague's Pipit

701 Cinclus mexicanus unicolor....

Dipper

71)- Oreoscoptes montanus.........

Sage Thrasher

(11:) Mimus polyglottos polyglottos..

Mockingbird

illin ilimus polyglottos leucopterus.

IVestern Mockingbird

int Dumetella carolinensis......... Catbird

io:) Toxostoma rufum............

Brown Thrasher

iuti Toxostoma longirostre sennetti.

Sennett's Thrasher

707 Toxostoma curvirostre curvi-

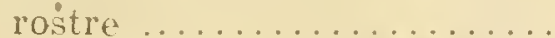

Curve-billed Thrasher

707ı Toxostoma curvirostre palmeri.. Palmer's Thrasher

$70 \Omega$ Toxostoma bendirei.

Bendire's 'Thrashęr

709) Toxostoma cinereum cinereum. .

San Lucas Thrasher

709 'Toxostoma cinereum mearnsi...

Mearns's Thrasher

710 Toxostoma redivivum

California Thrasher

711 Toxostoma lecontei lecontei...

I.econte's 'Thrasher' 

1910

I.U. U.

No.

Price

Per

711" Toxostoma lecontei arenicola...

Egg

Desert Thrasher

712 Toxostoma crissale..........

Crissal "Thrasher"

713 Heleodytes brunneicapillus

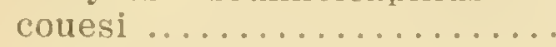

Cactus Wren

713 ॥ Heleodytes brumneicapillus bry-

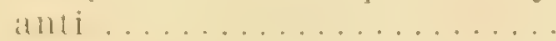

Bryant's Circtus Mren

Tlih Ireleodytes brumueicanillus affi-

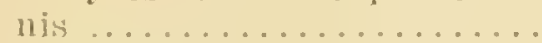

Sinn Lurras Cilctus Wren

715 Salpinctes obsoletus obsoletus.

Rock IVren

715 Salpinctes obsoletus pulverius. . Sinn Nicolis Rock Wreu

716 Salpinetes guadeloupensis..... Guadalupe Rock IV ren

717 Catherpes mexicanus albifrons. II hite-throited Wren

717 Catherpes mexicanus comspersus Cinnon Wren

7176 Catherpes mexicanus punctulat-

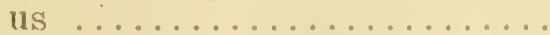

Dotted Canon Wren

2.50

1.50

71s T'hryothorus ludovicianus ludovicianum ...............

Carolina Wren

718 'Thryothorus ludovicianus mianincnsis .................

Florida IVren

7186) Thryothorus ludovicianus lomi-

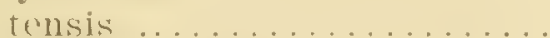

Lomita Mren

719 Thryomanes bewicki bewicki... Bewick's 11 ren

719" 'Thryomanes bewicki spilurus... Vigors's 11 ren

719h Thryomanes bewicki bairdi. . . . Baird's Wren

719. 'Thryomanes bewicki cryptus... 'l'exas IIren

710, Thryomanes bewicki charienturus ................

Gan Diego 11 ren

712 . Thryomanes bewicki calophonus Sieattle IV ren

719.1 Thryomanes leucophrys....... Sill Clemente IVren

720. Thryomanes brevicauda....... Giradalupe IVren

721 Troglodytes aëdon aëdon...... House IVren 

No.

Per

721" Troglodytes aëdon parkmani...

Egg

Western House Wren

722 Nannu's hiemalis hiemalis....

Winter Wren

722" Nannus hiemalis pacificus.....

Western Winter Wren

722h Namnus hiemalis helleri.......

Kadiak Winter Wren

;":⿱ Nibulls alascensis...........

Alaska Wren

F2:1 Nannus meliger.

Aleutian Wren

724 Cistothorus stellaris...........

Short-billed Marsh Wren

7:5 Telmatodytes palustris palustris

Long-billed Marsh Wren

725a Telmatodytes palustris paludicola ................... $\quad .10$

'Tule Wren

$725 b$ Telmatodytes palustris griseus..

Worthington's Marsh Wren

$725 c$ Telmatodytes palustris plesius.

Western Marsh Wren

725d Telmatodytes palustris iliacus. .

Prairie Marsh Wren

7:5e Telmatodytes palustris mariana

Marian's Marsh Wren

726 Certhia familiaris americana...

Brown Creeper

726 " Certhia familiaris albescens....

Mexican Creeper

726h Certhia familiaris montana....

Rocky Mountain Creeper

726c Certhia familiaris occidentalis..

California Creeper

726d Certhia familiaris zelotes...... Sierra Creeper

727 Sitta carolinensis carolinensis.. $\quad 1.00$

White-breasted Nuthatch

$727 \|$ Sitta carolinensis aculeata.....

Slender-billed Nuthatch

727h Sitta carolinensis atkinsi......

Florida White-breasted Nuthatch

727. Sitta carolinensis nelsoni..... 2.25

Rocky Mountain Nuthatch

727d Sitta carolinensis lagunæ......

San Lucas Nuthatch

728 Sitta canadensis............ 1.50

Red-breasted Nuthatch

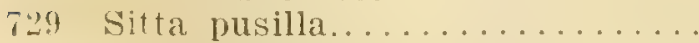

Brown-headed Nuthatch

730 Sitta pygmaa pygmæa........

Pygmy Nuthatch 

1910

A.O.U.

No.

$730 a$ Sitta pygmæa leuconucha.....

White-naped Nuthatch

731 Bæolophus bicolor............

Tufted Titmouse

732 Bæolophus atricistatus atricris-

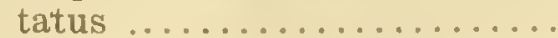

Black-crested Titmouse

$732 a$ Brolophus atricistatus sennetti. Sennett's Titmouse

733 Bæolophus inornatus inornatus. Plain Titmouse

733" Bæolophus inornatus griseus... Gray Titmouse

7336 Bæolophus inornatus cineraceus Ashy Titmouse

734 Bæolophus wollweberi........ Bridled 'Titmouse

735. Penthestes atricapillus atricapil-

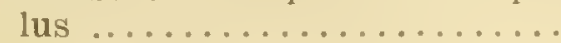

Chickadee

735a Penthestes atricapillus septentri-

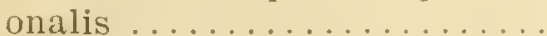

Long-tailed Chickadee

7356 Penthestes atricapillus occidentalis ................

Oregon Chickadec

735 c Penthestes atricapillus turneri.. Yukon Chickadee

736 Penthestes carolinensis caroli-

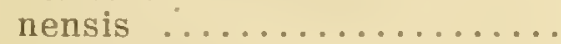
Carolina Chickadee

736 $\because$ Penthestes carolinensis agilis... Plumbeous Chickadee

7367 Penthestes carolinensis impiger. Florida Chickadee

737 Penthestes sclateri... Mexican Chickadee

738 Penthestes gambeli gambeli... Nountain Chickadee

738 a Penthestes gambeli baileyæ... Bailey's Mountain Chickadee

739 Penthestes cinctus alascensis... Alaska Chickadec

740 Penthestes hudsonicus hudsoni-

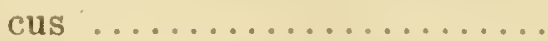
Hudsonian Chickadee.

740 a Penthestes hudsonicus littoralis. Acadian Chickadee

741 Penthestes rufescens rufescens. Chestnut-backed Chickadec

741a Penthestes rufescens neglectus. California Chickadee

7417 Penthestes rufescens barlowi... Barlow's Chickadee
Price

Per

ligg

3.00

1.25

.55

2.25

3.50

2.00

4.00 

1910

A.O.U.

No.

Price

Per

743 Psaltriparus minimus minimus..

Bush-Tit

743凡 Psaltriparus minimus californi-

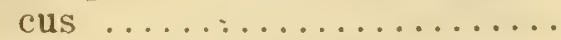

California Bush-Tit

7436 Psaltriparus minimus grindæ... Grinda's Bush-Tit

744 Psaltriparus plumbeus......... Lead-colored Bush-Tit

745 Psaltriparus melanotis lloydi... Lloyd's Bush-Tit

746 Auriparus flaviceps flaviceps... Verdin

746، Auriparus flaviceps lamproceph-

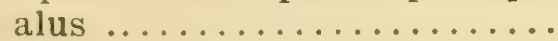

Cape Verdin

742 Chamæa fasciata fasciata...... Wren-Tit

742 ॥ Chamæa fasciata henshawi.... Pallid Wren-Tit

7426 Chamæa fasciata phæa........

Coast Wren-Tit

7420 Chamæa fa'sciata rufula........ .25

3.00

Ruddy Wren-Tit

747 Acanthopneuste borealis.......

Kennicott's Willow Warbler

748 Regulus satrapa satrapa.......

Golden-crowned Kinglet

748 ، Regulus satrapa olivaceus..... 6.50

Western Golden-crowned Kinglet

749 Regulus calendula calendula.... 8.00

Ruby-crowned Kinglet

749 a Regulus calendula grinnelli....

Sitka Kinglet

$749 h$ Regulus calendula obscurus.... Dusky Kinglet

751 Polioptila cærulea cærulea..... .50

Blue-gray Gnatcatcher

751r Polioptila cærulea obscura.....

Western Gnatcatcher

752 Polioptila plumbea.......... 1.00

Plumbeous Gnatcatcher

753 Polioptila californica.........

Black-tailed Gnatcatcher

754 Myadestes townsendi.........

'Townsend's Solitaire

755 Hylocichla mustelina........ $\quad .15$

Wood Thrush

756 Hylocichla fuscescens fuscescens $\quad .30$

Veery

756\% Hylocichla: fuscescens salícicola.

IVillow Thrush

757 Hylocichla aliciæ aliciæ.......

Gray-cheeked Thrush 

1910

A.O.U

Price

No.

Per

7570 Hylociclna alicize bicknelli.....

20.00

Bicknell's Thrush

758 Hylocichla ustulata ustulata... $\quad .25$

Russet-backed 'Thrush

758 " Hylocichla ustulata swainsoni.. .50

()jire-backed 'Thrush

759 Hylocichla guttata guttata.....

Mlaskal Lemit 'Thush

75.9 Hylocichla guttata auduboni...

Audubon's Hermit 'Thrush

759h Hylocichla guttata pallasi..... 1.00

II rmit 'I'hrush

75.9e Hylocichla guttáta nana...... 2.50

D) wrt' Hermit 'T'hrush

7594 Ifylociclila guttata slevini.... 3.50

Monterey Hemit 'Thrush

7596 Hylocichla guttata sequoiensis. 2.50

Sierral Hermit 'Thrush

| 7600| 'purdus musicus............ .50

leed-winged 'Thrush

ili. Planesticus migratorius migratorill . . . . . . . . . . .

Robin

761" Planesticus migratorius propin$[11 u ; \ldots \ldots \ldots \ldots \ldots \ldots \ldots$

Western Robin

761l Planesticus migratorius achrus terus .................. 25

Southern Robin

762 Planesticus confinis......... 4.51

San Lucas Robin

76.3 Lxoreus nævius nævius...... 3.25

Varied Thrush

$76:$ Ixoreus nævius meruloides.... 4.50

Northern Varied 'Thrush

1764] Cyanosylvia suecica robusta...

2.00

Siberian Red-spotted Blue-throat

765 Saxicola cenanthe nenanthe.... .25

Wheatear

765 a Saxicola œnanthe leucorhoa... 4.50

Greenland Wheatear

766 Sialia sialis sialis.......... .15

Bluebird

766 " Sialia sialis fulva.......... 3.50

Azure Bluebird

767 Sialia mexicana occidentalis.... .15

Western Bluebird

767 . Sialia mexicana bairdi......... .15

Chestnut-backed Bluebird

767 , Sialia mexicana anabelæe...... 2.25

San Pedro Bluebird

768 Sialia currucoides.............

Mountain Bluebird 


\section{CLASSIFIED ADVERTISEMENTS}

DR. IVILLIAM ROUNDS, Nature, Student. Lock Box 144, Fort Worth, 'Tex.

JAKE ZEITLIN, Scientific Collector and Naturalist. 905 Bryan Ave., Fort Worth. 'Texas.

D. V. HEMBREE, Roswell, Georgia, U. S. A. Taxidermist. Bird skins and Mounted Birds for sale and exchange.

BIRDS, ANIMALS and Heads mounted. Skins made into rugs. M. B. Wheeler, Taxidermist, Randolph, N. Y.

I WAN'T TO PURCHASE books on California and Western birds. Emerson stoner, Box 44, Benicia, California.

FOl: SALE and EXCHANGE-Personally collected sets of eggs and nests, also Bird skins. John Lorang, Genesee, Ilitho.

EGGS IN SETS with data to exchange on the basis of values set in this catalog. Emerson Stoner, Box $4 t 4$, Benicia, California.

FOR SAI, - Write for a list of a few personally collected sets from Nova Scotia. Hobie WV, Tufts, Wolfville, Nova Scotia.

EXCHANGES, SALES A field Ornithologist and Oologist for very many vears. It. M. Carryl, No. 1 Princeton St. Nutley, N. J.

BIFDS and ANIMALS mounted. Skins tanned. Write for price list. Ramon Grahain, Taxidermist, 3722 Ave. J. Poly Fort Worth, lexas.

EXCHANGE-Texas birds' eggs in sets, Bird and animal Skins, Curios. Natural History specimens. Ramon Graham, 3722 Ave. J. Poly, Ft. Worth, 'l'exas.

I have Choice Full Sets of A. O. U. Nos, toa, 40t, 583, 730, 738, 759a, 768 and wish sets of sparrows, and grouse and quail in exchange. Fred Dille, Valentine, Nebraska.

OGG and WI'TLEY, Antler, North l)akotir. Collectors and "laxidermists of North American Birds. Supplies of ill kinds for sale or exchange-Nests, loggs, Mounted Birds and Bird Skins.

WRIGH M. PIERCE, Clarmont, Calif., has a general collection of North American birds eggs and desires exchanges with reliable collectors. Want specially sets with nests of perfect preservation.

DESIRED-Correspondence with collectors on all matters pertaining to hirds, their nests and eggs, and Bird Plotography. Fxchange of Photocriplis and specimens. Dr. A. G. Prill, sicio. Oregon.

WANTIOD-First class sets with full data of almost any Speckled Eggs, forcign or North American. I also want line photographs of nests and eggs. Kitrl A. Pember, Woodstock, Vt., U.S.A.

FOR SALE-Bright showey Butterllies in papers from South America, India and Africa, suitable for Trays, pictures, etc: also many natives. A. J. Potter, East Killingly, Conn.

IV ANTED FOR CASH-Sets of Eggs with full data of Raptores of the world. excepting the commonest North American and European species. H. Kirke
Swann, Thorncombe, Lyonsdown. New Barnet, Herbs, England.

I have a lot of Ornithological books and periodicals for disposal, but haven't laad time to issue lists. If there is any book wanted, please communicate and a favorable price will be given. Fred Dille, Valentine, Nebraska.

for my private collections I will nurchase desired specimens in Ornithology, Oology, Mamology, and Conchology. Limited exchange will also be considered in these lines. Albert 1. Ganier, "2507 Ashwood Ave. Nashville. 'l'ennessee.

COLE BIOS, Naturalists, Nevada. lown. Birds, Nests, Eggs, Skins

WAN'PED-To correspond with collectors who are just starting a collection of egos, who can use many common ones from other localities, would be glad to correspond with any who can use any species froni the central west, Martin S. Paulson, Nevada, Iowa.

KENNE'l L SKINNER, Brooklands Estate Office, Weybridge, England, specializes in the eggs of the FRINGIL. LIDAE, and wishes to obtain eggs from all parts of the world, by purchase or exchange. Having excellent opportunities of securing other rood material he will be pleased to co-operate witli collectors who will assist him.

WANTED-I'o correspond with collectors in the South. West and North who can use sets common to this locality. Want Raptores, Anseres and lingillidae. Enclose your list of duplicates with your first letter, all letter. answered. John L. Cole, Nevada, Iowa.

EGGS-Desirable single Eggs-Have amassed a quantity of Maynard-Series Singles. Many, most rare. Yours, on attractive terms. Write me. New idea in collecting. Charming Bird Photographs taken in five states. Some depict facts new to science. IVrite for prices, with stamp. P. B. Peabody, Blue Rapids, Kansas

H. ARDEN EDTVARDS, Field Oologist.-Eggs of the world for compar ative work. Offers in exchange and desires only specimens of the highest class. Sets perfect with nest material Special desiderata, Paridae, Macroshire, Corvildae and Gallinae. Address H. Archer Edwards, Los Angelés, Cal., [?. S. A., IR. D. 1, Box 284.

WANTUD-Any A. O. U. sets not repsented in my collection, for which ofter cash or tine exchange Want list includes No. \&, 131, 180, 204, 205 215. Many shore Birds, $354 \mathrm{~b}, 417 \mathrm{a}$ 118a: many species IVoodpecker, Jay. sparrows and Warblers. Many common kinds needed. First class and positively athentic only accepted. I C. Price, Gritnt Park, Ill.

IAIDHRMY-Specimens scientiflcally mounted. Special attention given to posing. Casework and backgrounds, hest of workmanship. Groups and collections mounted finest museum style. I specialize on basework which you can not duplicate elsewhere. Send me Four next specimens and be convinced. Price list on request. L. IV. Speer. 'Taxidermist, Sac City, Iowa. 
Fellow Bird-Men:

To faithfully reproduce the delightful glimpses every Ornithologist has of birds in the field; to artfully idealize the bird without neglecting its scientific structure and color; to portray the living bird in its most natural and therefore most charming aspects -.- to these tasks have I set my hand and brush.

\section{Very truly yours, GEORGE MIKSCH SUTTON, Carnegie Museum, Pittsburgh.}




\section{The Museum of Comparative Oology} OF

\section{SANTA BARBARA, CALIFORNIA FOUNDED JANUARY 31st, 1916}

\section{THE COOPERATIVE MUSEUM}

The IV. C. O is the pioneer institution in the realm of Oölogical Research, and the only chartered public musemm maring exclusive attention to the study of birds, their nests, and eggs.

The IM. C. O. inaintains a collection of hirds' eges which aims at cosmopolitan completeness.

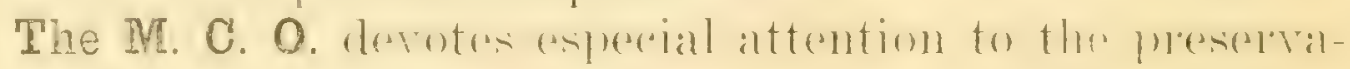
tion of nests-with-eges, and has perhaps the finest collection of birds' nests in existence.

The IM. C. O. is the official home and depository of a wohld wisk followship of oblogists. known as The Members of the Miseum of Comparative Oology. This ainlel now has upwards of 200 members who represent 35 states and 29 foreign comtries.

The M. C. O. publishes two journals, one a quarterly. A.mun a The Journal of the Musenm of Comparative Oology, devoted to the interests of the Institution and of it: Wrmbers, and which deals with matters comnected with

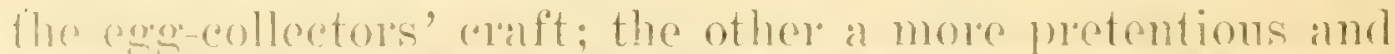
wholly illudritul ammul. The Comparative Oologist, which reals with the seientific aspects of oologre or (mmhorlies the most interesting results of field work.

If you take the eollecting of birds' agess seriously, or if ron desire to see the ultimate onteome of resenroh in this fascinating field, you may cale to "belong." Apprentice Wembels (ages 12 to 17 ) wp to the limit of 100 a a also welcomol and instructed.

for fouther information address.

\section{The Director Museum of Comparative Dology, Santa Barbara, California}




\section{BENJAMIN HOAG GARFIELD, NEW YORK}

\section{Oologists Supplies}

Tools - Egg Drills - Blow Pipes

\section{BOOKS}

\section{MAGAZINES}

\section{SPECIMENS}

\section{BENJAMIN HOAG}

GARFIELD, NEW YORK 


\section{NATURAL HISTORY SUPPLIES FOR TAXIDERMISTS}

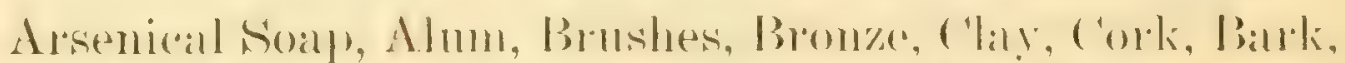

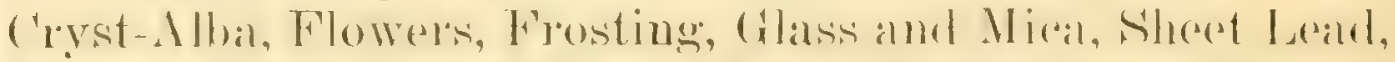

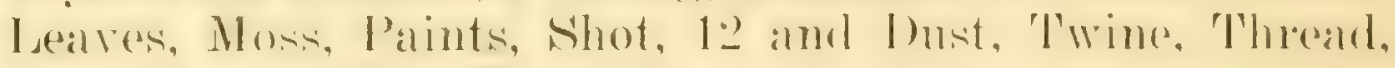

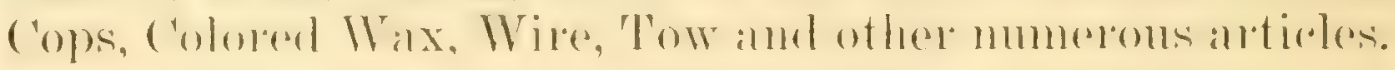

Bird Stands and Mounts, a Specialty

\section{Manufacturer of Paper Mache Skulls}

Felt, Best Quality; Brown Duck for lining, Heary Sheet Wadding.

OOLOGIST'S AND ENTOMOLOGIST'S SUPPLIES

\section{For Museums and Schools}

Mounted Birds, Animals, Fish, Reptiles

Bird Skins and Eggs

Land and Marine Curios

\section{TAXIDERMISTS}

We do all varriety of work in this line. Orders solicited.

\section{BOOKS}

Results in Taxidermy, A Guide to Positions

140) Ilalf 'Tone Illustrations showing hundreds of specimens. Assistance to Naturalists and interesting to all. Lists of other books furnished.

Send For Our Price List.

GLASS EYES

Imported and Domestic

Over 600 Varieties in Stock. Best Makes Obtainable.

\section{THE FRANK BLAKE WEBSTER CO. HYDE PARK, MASS.}




\section{FRANK 0. PATTON}

NATURALIST

South Dakota Birds, Nests, and Eggs.

ARTESIAN, SO. DAKOTA

\section{OSCAR E. BAYNARD NATURALIST \\ OOLOGY \\ MY \\ SPECIALTY}

.Plant City,

Florida
WILL COLLECT TO ORDER SPECIES FROM THIS LOCALITY

Want especially ANSERES, and RAPTORES

Send for my Exchanye list

JOHN L. COLE, Nevada, Iowa

\section{The Jacobs Museum of} Applied Oology

An institution for the Study of Relationship of Birds, desires sets of eggs containing abnormally marked or unmarked, albinistic or in anywise malformed specimens. Only those with good data wanted to further our studies of Oological Abnormalities for a more complete revision of the work published by J. Warren Jacobs in 1898.

When you have anything of this nature please write us.

J. Warren Jacobs, Director, Jacobs Museum of Applied Oology Waynesburg, $\mathrm{Pa}$.

\section{ARIZONA}

Can always supply a few sets from this state.

Write:

\section{EDGAR M. LeBARON Mesa. Arizona}

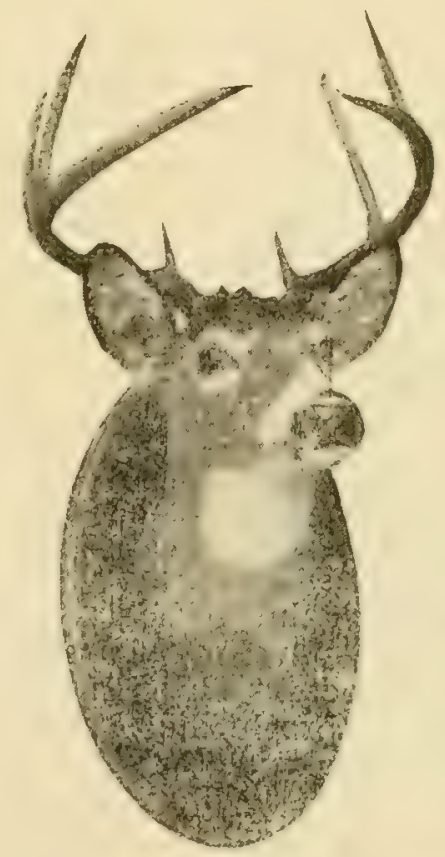

\section{MOUNTING} TO ORDER

BIRDS ANIMALS GAME HEADS FISH. FUR RUGS .TAXIDERMIST SUPPLIES

PAPER HEADS for DEER, ELK, MOOSE.

OPEN MOUTH HEAI'S For RUG WORK; EYES, SHIELDS, ETC. SEND FOR

Mounted Rugs, Heads, etc., for sale; also Unmounted Scalps, and Animal Skins for sale.

11. J. HOFMAIN, Taxidermis t 939 Gates Ave., BROOKLYN, N. Y. 


\section{DEAN'S}

\section{NATURAL SCIENCE ESTABLISHMENT}

Animals, Birds' Skins, Eggs, Nests, Minerals, Fossils, Shells, Indian \& War Relics, Curios, Butterflies, Stamps, Coins

Naturalists, Taxidermists and Biological Supplies.

Taxidermy in all branches.

Free Public Museum R. A. DEAN

103 E. State St., ALLIANCE, OHIO

\section{ISAAC E. HESS (PHILO-ILLS)}

In the market for clear photographs of Mississippi Valley Hirds, only adult bird photos desired with right to illustrate.

Correspondence solicited with museums interested in entire local collection nests and eggs of lllinois Birds-105 species in excellent types and markings.

Victor L. Smith Earl R. Smith

\section{COLLECTORS} of

Birds Eggs, Stamps, Coins, Mounted Specimens and Curios of all kinds.

Box 119

MICHIGAN

'THE OOLOGISTS' RECORD

A Quarterly Magazine devoted to the advancement of Oology in all parts of the World

SUBSCRIPTION, J shillings (\$1.20) per year.

First volume 1920. Back numbers will be supplied, as long as they last, at subscription rates.

The leading oologists in all countries subscribe to this live paper and no keen student of oology anywhere can afford to be without it.

Published by Harrison and Sons, Jd., Printers in Ordinary to His Majosty, 45 St. Martin's Lane, London, W. C. 2, England

\section{THE OOLOGISTS' EXCHANGE AND MART}

Founded in 1919 and supported from the start by the best known collectors everywhere.

Its Founder and Editor holds that, next to the eggs one collects one. self, the best are those received at first hand from trusted correspon. dents. Its chief purpose, therefore, is to bring all collectors of good standing into direct touch with each other. This purpose has been fully achieved, and the brotherhood thus formed is splead over the whole world.

New subscribers must be nominated by an existing subscriber, or may be admitted by the Editor on his being satisfied as to their standing and bona fides. No dealers are admitted as subscribers.

SUBSCRIPTION 7s. Gi $(\$ 1.80)$ per year, which includes:

(1) The four quarterly issues of "The Oologists. Record" (alone worth $\$ 1.20$ ).

(2) The four quarterly issues of "The Oologists' Exchange and Mart."

(3) The inclusion of the Subscriber's name and address in "I'he $O$. E. and $M^{\prime \prime}$. on joining, and again each year, on renewal of subseription.

(4) The right to one free advertisement of $25 \mathrm{worls}$ in "The $\mathrm{O}$. E. and $\mathbf{M}$." each year. (Extra advertisements, or extra wolds, are charged for at ? cents per word). 


\section{SIMPLEX SUET HOLDER}

The simplest, most effective and inexpensive apparatus for attractive birds about your home, rendering them aid in the trying times of severe winter weather, as well as throughout the year.

The Simplex Suet Holder has passed the test and proved to be the most satisfactory method of offering suet to the birds. Iany species not usually expected to thus feed have been found to accept the invitation that the Simplex Suet Holder extends. It eliminates waste, while presenting the suet to the birds in such a manner that they can secure the last bit, is convenient and quick filling and adjusting and is unobstructive and not displeasing to the eye.

While costs of material, like practically everything else, have soared, the price of the Simplex Suet Holder has been maintained at pre-war rates. That the birds and bird lovers may benefit, we offer the simplex suet Holders at thirty-five cents each, three for one dollar, three dollars per dozen, postpaid.

The winter is the important time to get the Suet Holders out, although the birds will feed from them, more or less, throughout the year. Don't fail to send for at least three of the Simplex Suet Holders and help the birds of your locality to winter through.

SIMPIEX BIRD APPARATUS COMPANY, DEMAREST, NEW JERSEY.

\section{CHOICE EGGS IN SETS and ORNITHOLOGICAL PUBLICATIONS}

The undersigned desires correspondence with those having desirable sets of eggs or ornithological publications for exchange or disposal, and those who are seeking such material. Would also be interested in good bird photographs.

Those having for disposal first class, authentic sets of the rarer gulls, petrels, shore birds, grouse, ptarmigan, doves, hawks, owls, woodpeckers, Frosted Poor-will, Sennett's and Pacific $\mathrm{N}$ ig h t h a w ks, hummingbirds, Lower California Flycatcher, Large-billed Wood Pewee, San Lucas Flycatcher, Beardless Flycatcher, horned larks, jays, Rio Grande and Southern Meadowlarks, Sennett's Orioles, Grosbeaks, sparrows, finches, swallows, vireos, warblers, White and Swinhoe's Wagtails, Mearn's and Desert Thrashers, wrens, liocky Mountain Creeper, nuthatches, titmice, chickadees, Coast and Ruddy Wren-tits, Kennicott's Willow Warbler, 'Thrushes, Siberian Red-spotted Blue-throat, wheateaters, Chestnut-backed and San Pedro Blucbirds, will do well, before disposing of them to communicate with

\section{B. S. BOWDISH,} Demarest, N. J. 


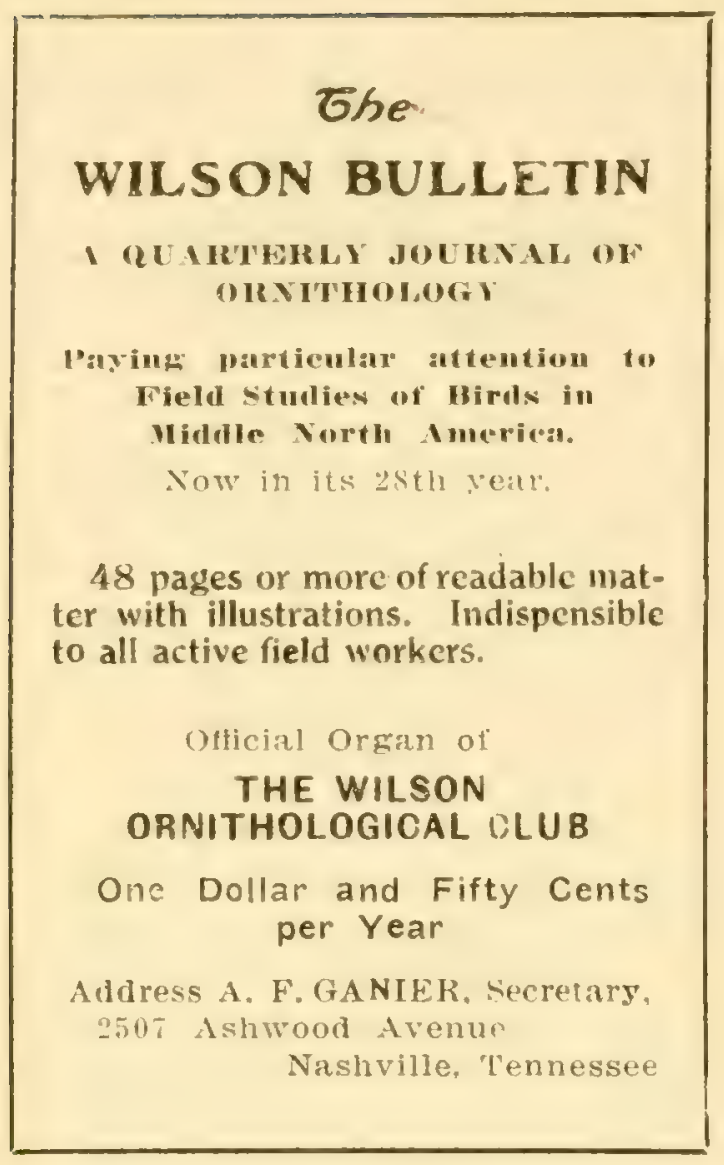

\section{COLE BROS.}

Nevada, lowa

\section{NATURALISTS}

\section{BIRDS}

\section{NESTS}

\section{EGGS}

\section{SKINS}

\section{COLLECTING}

\section{Birds and Eggs}

IS AN INTERESTING, BENEFICIAL AND

PROFITABLE OUT-OF-DOORS HOBBY.

$$
\mathcal{T} R \quad Y \quad I T !
$$




\section{BIRDS ECGS FOR EXCHANGE}

I have the largest list of duplicate eggs for exchange in North America.

I have sources of supply UNEQUALED by any other collector in North America.

For Rare Specimens my opportunities are GREATER than any other Oologist in North America.

My Private Collection is the LARGEST collection of North American Eggs, outside of the U. S. National Museum, in the World.

I am always in the market, to buy or exchange, for specimans of Eggs or Skins of Birds desired by me, and am willing to give the highest price in Cash or the best exchange for what I want.

\section{R. M. BARNES LACON, ILL.}





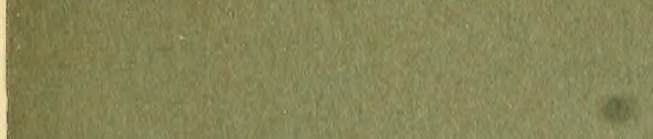


